\title{
Modeling experiments in helioseismic holography
}

\author{
Dissertation \\ zur Erlangung des mathematisch-naturwissenschaftlichen Doktorgrades \\ "Doctor rerum naturalium" \\ der Georg-August-Universität Göttingen \\ im Promotionsprogramm PROPHYS \\ der Georg-August University School of Science (GAUSS)
}

\author{
vorgelegt von \\ Dan Yang \\ aus Hebei, China
}

Göttingen, 2018 
Betreuungsausschuss

Prof. Dr. Laurent Gizon

Max-Planck-Institut für Sonnensystemforschung und

Institut für Astrophysik, Georg-August-Universität Göttingen, Deutschland

Prof. Dr. Stefan Dreizler

Institut für Astrophysik, Georg-August-Universität Göttingen, Deutschland

Dr. Aaron C. Birch

Max-Planck-Institut für Sonnensystemforschung, Göttingen, Deutschland

Mitglieder der Prüfungskommision

Referent: Prof. Dr. Laurent Gizon

Max-Planck-Institut für Sonnensystemforschung und

Institut für Astrophysik, Georg-August-Universität Göttingen, Deutschland

Korreferent: Prof. Dr. Stefan Dreizler

Institut für Astrophysik, Georg-August-Universität Göttingen, Deutschland

Weitere Mitglieder der Prüfungskommission:

Dr. Aaron C. Birch

Max-Planck-Institut für Sonnensystemforschung, Göttingen, Deutschland

Jun.-Prof. Dr. Christoph Lehrenfeld

Institut für Numerische und Angewandte Mathematik, Georg-August-Universität Göttingen, Deutschland

Prof. Dr. Hardi Peter

Max-Planck-Institut für Sonnensystemforschung, Göttingen, Deutschland

Prof. Dr. Ramin Yahyapour

Gesellschaft für wissenschaftliche Datenverarbeitung mbH Göttingen und Institut für Informatik, Georg-August-Universität Göttingen, Deutschland

Tag der mündlichen Prüfung: 03. Dezember 2018 


\section{Bibliografische Information der Deutschen Nationalbibliothek}

Die Deutsche Nationalbibliothek verzeichnet diese Publikation in der Deutschen Nationalbibliografie; detaillierte bibliografische Daten sind im Internet über http: //dnb . d-nb . de abrufbar.

ISBN 978-3-944072-67-8

uni-edition GmbH 2019

http://www.uni-edition.de

(C) Dan Yang

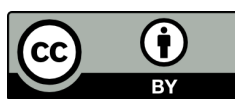

This work is distributed under a

Creative Commons Attribution 3.0 License

Printed in Germany 



\section{Contents}

\begin{tabular}{ll}
\hline Summary & 7
\end{tabular}

1 Introduction 9

$1.1 \quad$ Solar oscillations $\ldots \ldots \ldots \ldots$. . . . . . . . . . . . . . 9

1.2 Helioseismology $\ldots \ldots \ldots \ldots \ldots$. . . . . . . . . . . . . . 11

$1.2 .1 \quad$ Global helioseismology . . . . . . . . . . . . . . . . 11

1.2 .2 Local helioseismology . . . . . . . . . . . . . . . . . . . . . . . . . . . 12

1.3 Helioseismic holography . . . . . . . . . . . . . . . . . . . . . . . . . . . . . . . . .

1.4 Results contained in this work . . . . . . . . . . . . . . . 15

2 Paper I: Ghost images in helioseismic holography? Toy models in a uniform $\begin{array}{ll}\text { medium } & 17\end{array}$

2.1 Abstract . . . . . . . . . . . . . . . . . . . . . . 17

2.2 Introduction . . . . . . . . . . . . . . . . . . 17

2.3 Source-sensitivity kernels . . . . . . . . . . . . . . . . . . . . . . . . . . . . . . . .

2.4 Toy model: Waves in a homogeneous medium . . . . . . . . . . . . 21

2.5 Results . . . . . . . . . . . . . . . . . . 21

2.5 .1 Source-sensitivity kernels at $3 \mathrm{mHz}$. . . . . . . . . . . . 21

$2.5 .2 \quad$ Kernels averaged over frequency . . . . . . . . . . . . . . . . 24

2.5 .3 Dependence of the spatial resolution on the coverage . . . . . . 26

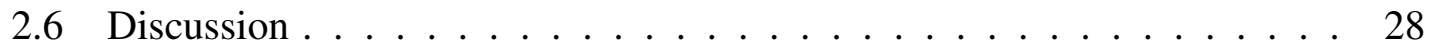

2.6 .1 Ghost images in the egression . . . . . . . . . . . . 28

2.6 .2 The ingression and the PB hologram . . . . . . . . . . . . . . . . 29

2.6 .3 Application to stereoscopic helioseismology. . . . . . . . . . . . 29

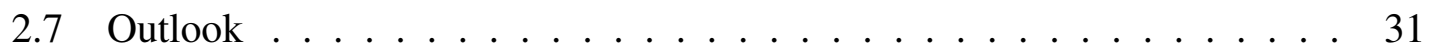

3 Paper II: Signal and noise in helioseismic holography 3

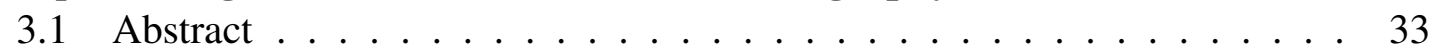

3.2 Introduction . . . . . . . . . . . . . . . . . 33

3.3 Reduced wave equation . . . . . . . . . . . . . . . . . . . . . . . 34

3.4 Holographic image intensity . . . . . . . . . . . . . . . . . . 36

$3.5 \quad$ First-order perturbations with respect to a reference solar model . . . . 38

3.5 .1 Perturbations to the wavefield . . . . . . . . . . . . . . . . . . . . . 38

3.5 .2 Perturbations to the PB integral . . . . . . . . . . . . . 39

$3.5 .3 \quad$ Perturbations to the image intensity . . . . . . . . . . . . . . 39

3.5 .4 Choice of the source covariance . . . . . . . . . . . . . . 41 
3.6 Noise . . . . . . . . . . . . . . . . . . . . . . . . . . . . . 41

3.7 Average over frequencies . . . . . . . . . . . . . . . . 42

3.8 Example computations . . . . . . . . . . . . . . . . . . . 43

3.8 .1 Reference Green's function. . . . . . . . . . . . . . . . . . . 43

3.8 .2 Sound-speed kernels . . . . . . . . . . . . . . . . . . . . . . . . . . . . . . . . . 44

3.8 .3 Signal . . . . . . . . . . . . . . . . . . . . . . . . . . . . . . . . . 45

3.8 .4 Noise . . . . . . . . . . . . . . . . . 46

3.8 .5 Signal-to-noise ratio . . . . . . . . . . . . . . . . 47

3.9 Conclusion $\ldots \ldots \ldots \ldots \ldots \ldots$

4 Imaging solar subsurface flows using diffraction-limited seismic holography 53

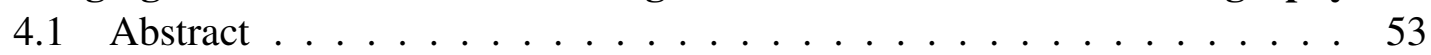

4.2 Introduction . . . . . . . . . . . . . . . . . . 53

4.3 Helioseismic holography to measure flows . . . . . . . . . . . . . . . 54

4.4 Quadrant geometry . . . . . . . . . . . . . . . . 55

4.5 Holographic correlation . . . . . . . . . . . . . . . . . 55

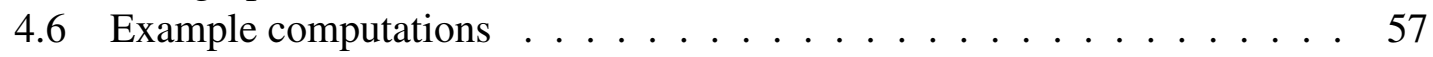

4.6 .1 Zonal flow . . . . . . . . . . . . . . . . . . 57

4.6 .2 Meridional flow . . . . . . . . . . . . . . . . . . . 59

4.6 .3 Signal-to-noise ratio . . . . . . . . . . . . . . . . . . . 59

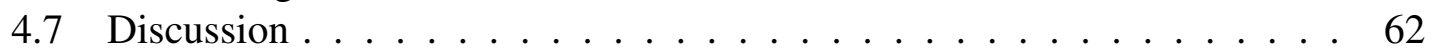

4.7 .1 Cross-talk between zonal flow and other scatterers . . . . . . . 62

4.7 .2 Outlook . . . . . . . . . . . . . . . . . . 63

$\begin{array}{lll}5 & \text { Future work } & 65\end{array}$

5.1 Far-side imaging . . . . . . . . . . . . . . . . 65

5.2 Resolving solar internal flows at the diffraction limit . . . . . . . . . . . 66

\begin{tabular}{lr}
\hline Bibliography & 67
\end{tabular}

\begin{tabular}{|ll|}
\hline A Computing PB integrals in spherical harmonic space & 77
\end{tabular}

A.1 Notations . . . . . . . . . . . . . . . . . . . 77

A.2 Spherical harmonics . . . . . . . . . . . . . . . . . 77

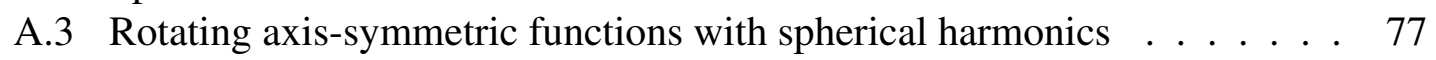

A.4 $\quad$ Source-sensitivity kernel in spherical harmonic space . . . . . . . . . . . 78

A.5 Noise in spherical harmonic space . . . . . . . . . . . . . . . . . 79

\begin{tabular}{lr}
\hline Scientific contributions & 81
\end{tabular}

\begin{tabular}{lr}
\hline Acknowledgements & 83
\end{tabular}

\begin{tabular}{lr}
\hline Curriculum vitae & 85
\end{tabular} 


\section{Summary}

This dissertation aims at developing a theoretical framework for the signal and noise in helioseismic holography which is mathematically rigorous and physically meaningful. A main focus of this dissertation is modeling the Porter-Bojarski (PB) integral, a wellestablished method used in the field of acoustics to locate subsurface sources and scatterers. We test and validate the potential application of the PB integral to probe the Sun's internal structure and dynamics.

In the first study, we compare the PB integral with the 'egression', the current imaging technique used in helioseismic holography, in a homogeneous medium. This proof of concept shows that the two imaging methods can locate subsurface sources of acoustic waves, which have a similar spatial resolution. However, the egression suffers from artificial signals located away from the source, whereas the PB integral does not. This suggests that the PB integral can potentially improve the current imaging capability of helioseismic holography.

The next study implements the PB integral for a realistic solar model, and a theoretical framework is developed to investigate its signal and noise. Solar oscillations are formulated into the solution of a scalar Helmholtz equation with the background soundspeed and density taken from a standard solar model, and are excited by a stationary and spatially uncorrelated random source function. We then apply the first-order Born approximation to relate scatterers such as sound-speed heterogeneities, density, and flows to PB integrals. The example computations show that PB holographic measurements are diffraction limited, i.e., the spatial resolution is half the local wavelength. We also investigate noise due to the random nature of wave excitation. We find large variations in both signal and noise at low frequencies due to contributions from individual long-lived modes of solar oscillations, and low signal-to-noise ratios for measurements above the frequency cut-off.

With the theoretical framework in hand, we then investigate the optimal flow-measuring strategy for helioseismic holography. Two different approaches are investigated and compared, the traditional method that measures directional phase shifts using pupils in a quadrant geometry (method \#1); a new method that correlates the estimated wave field at two nearby locations in the solar interior using all observed waves (method \#2). We find that method \#2 is consistently superior to the traditional method. Specifically, it reaches the diffraction limit of acoustic waves (half the local wavelength) and has a much higher signal-to-noise ratio than the traditional method. Furthermore, it is much less susceptible to the leakage from the solar surface. Therefore, we conclude that method \#2 will improve the imaging of solar subsurface flows using heliosemic holography, and hence should be used in future applications. 



\section{Introduction}

The solar convection zone is highly dynamic. Flows of various lifetimes and spatial scales interact with each other, which produce and maintain a magnetic field. The magnetic fields generated therein emerge to the surface due to magnetic buoyancy, and cause a wide variety of atmospheric eruptive phenomena (e.g., jets, flares, coronal mass ejections). Though we have numerous observations of the consequence of the Sun's dynamic interior, and a basic understanding of the causes, we have yet to comprehend and predict the evolution of these systems.

To-date, the only technique that allows a direct probe of the Sun's internal flows is helioseismology. Helioseismology aims to decipher the Sun's three-dimensional structure information which is encoded in the acoustic and surface-gravity waves observed at the surface. Despite two decades of continuous helioseismic observations from space-borne telescopes as well as ground-based global networks, however, a consistent and accepted picture of the structure and evolution of large-scale flows remains elusive in the Sun's interior. This is because we still lack adequate tools for a proper interpretation of helioseismic measurements. Therefore, a major task of helioseismology nowadays is to improve the tools used for data interpretation, in particular an accurate understanding of the wave propagation in the Sun. Additionally, efforts should be made to explore alternative flow-measuring strategies that are less susceptible to noise.

This dissertation intends to better understand the theoretical framework of one particular helioseismic technique known as helioseismic holography, particularly the relationship between signal and noise. In the following chapter, I will briefly introduce solar oscillations and provide a general review of various helioseismic techniques. I will then focus on the current achievements and limitations of helioseismic holography.

\subsection{Solar oscillations}

In this section, I will give a very brief introduction on solar oscillations. Various books are available for more details (see, e.g., Unno et al. 1989, Stix 2002, Aerts et al. 2010).

The Sun's turbulent convection excites solar oscillations in its near-surface layers (e.g., Goldreich and Keeley 1977, Stein and Nordlund 2001), which leads to ubiquitous intensity and velocity variations observed at the surface with periods near five minutes as first discovered by Leighton et al. (1962). This complex wave field can be characterized by three categories of modes based on their physical properties:

1. Pressure modes ( $\mathrm{p}$ modes) are acoustic waves with the pressure as the restoring force. The majority of $\mathrm{p}$ modes propagate in cavities defined by a lower and upper turning points. At the lower turning point, waves are refracted due to the increasing sound speed 


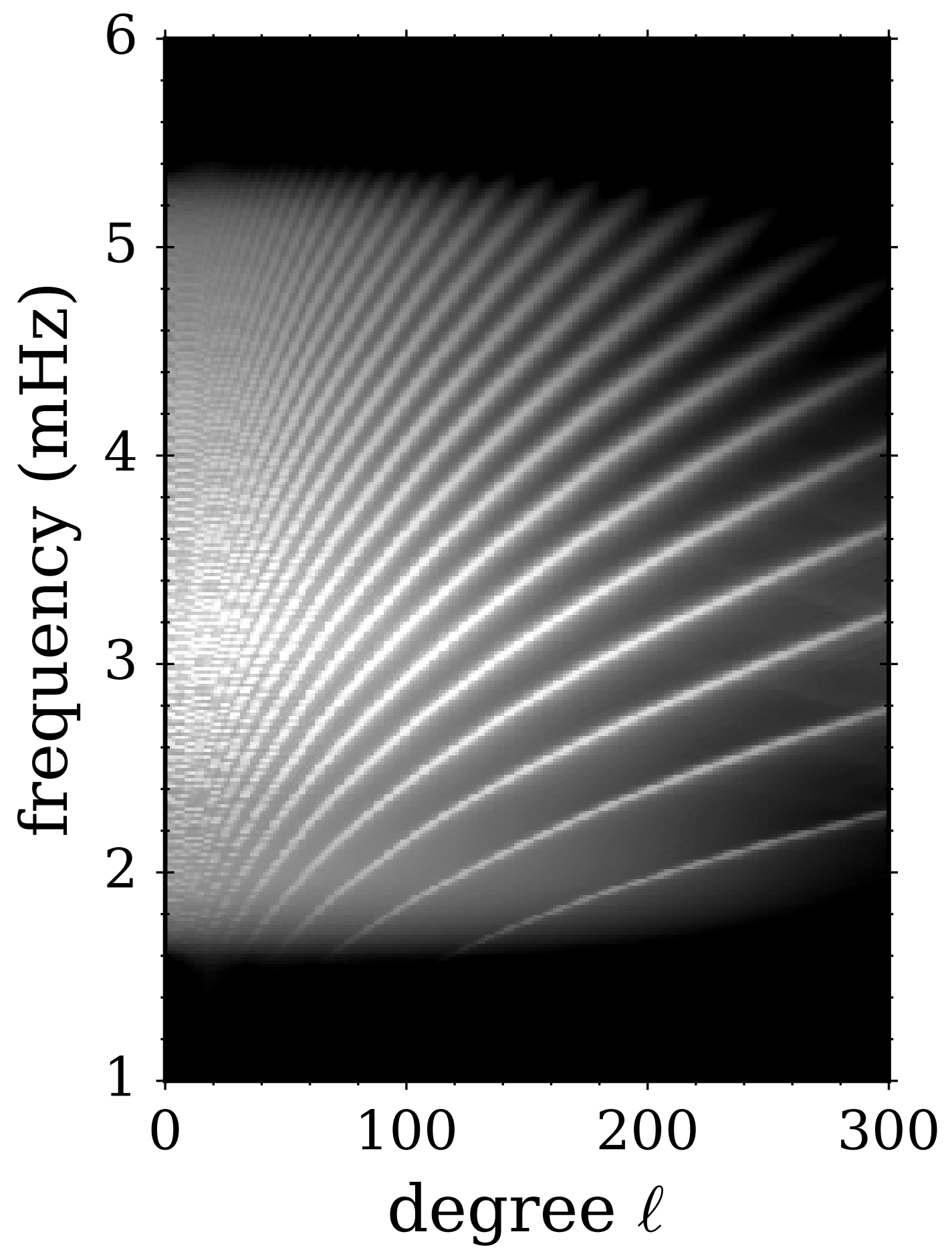

Figure 1.1: m-averaged power spectrum of solar $\mathrm{p}$ modes observed in line-of-sight velocity from HMI/SDO (courtesy of Zhichao Liang). The black/white indicates low/high wave power. 
towards the solar center. At the upper turning point, waves below $5.3 \mathrm{mHz}$ are reflected near the surface due to the sharp drop of density.

2. Surface gravity modes (f modes) behave very much like deep water waves; the restoring force is buoyancy. The observed f modes are mostly sensitive to the near-surface layer, and hence a good diagnostic of the structure therein.

3. Internal gravity modes (g modes) are confined in convectively-stable layers, i.e., the radiative zone; the restoring force is also buoyancy. g-modes are sensitive to the deep interior of Sun, where the structure is least constrained by p modes. The solar g modes, however, are evanescent in the entire convection zone, and as a result have very small amplitude at the surface (with an estimated upper limit of $10 \mathrm{~mm} / \mathrm{s}$ for a single mode, see Appourchaux et al. 2000). As a consequence, g modes are very difficult to detect, and, until today, the detection of g modes is still ambiguous (see, e.g., Fossat et al. 2017, Schunker et al. 2018).

The above modes are also known as the spheroidal modes, which have zero vorticity in the radial direction of the Sun (Aizenman and Smeyers 1977). The complementary of the spheroidal modes is the toroidal modes, which are horizontal motions with nonzero radial vorticities (see, e.g., Aizenman and Smeyers 1977, Unno et al. 1989). For a non-magnetic non-rotating star, the toroidal modes correspond to steady horizontal eddy motions, however, they become oscillatory ( $\mathrm{r}$ modes) once the star starts to rotate. $\mathrm{r}$ modes are predicted to have frequencies in the order of the rotation frequency; the restoring force is the Coriolis force. In the Sun, $r$ modes have long been just a theoretical conjecture. This situation, however, has changed with recent unambiguous detection of $\mathrm{r}$ modes on the Sun's surface and interior (see, e.g., Löptien et al. 2018, Liang et al. 2018a, Hanasoge and Mandal 2019). The detection of $r$ modes provides an additional way to probe the Sun, where we may learn more about the Sun's internal structure and dynamics.

\subsection{Helioseismology}

Helioseismology is the field of research that exploits the Sun's structural information contained in solar oscillations. This is achieved through minimizing the difference between observational properties of solar oscillations and their corresponding theoretical predictions. As such, an accurate understanding of wave propagation in the Sun, hereafter forward modeling, is required for interpretation of helioseismic measurements.

Based on the target spatial scales of the Sun's structure, helioseismic techniques are divided into two categories, global and local. In the following, I will briefly review these two categories of techniques.

\subsubsection{Global helioseismology}

Global helioseismology is based on the normal mode theory of solar oscillations, and is sensitive to the global structure of the Sun. For a spherically symmetric Sun, solar oscillations can be simplified as the solution of a linear wave equation, thereby forming an eigenvalue problem. Specifically, the displacement vector $\boldsymbol{\xi}$ of an oscillation mode satisfies

$$
\mathcal{L} \boldsymbol{\xi}_{n l m}=\omega_{n l m}^{2} \boldsymbol{\xi}_{n l m},
$$


where $\mathcal{L}$ is a linear operator that describes the wave propagation and $\omega_{n l m}$ denotes the eigenfrequency of a particular mode. Here, $n, l$, and $m$ are quantum numbers with $n$ describing the number of radial nodal points of the mode eigenfunction, and $l$ and $m$ as the number of nodal lines in the horizontal (both longitudinal and latitudinal) and longitudinal directions.

The eigenfrequencies of solar oscillations obey a variational principle; they are stationary with respect to small perturbations of the eigenfunction (Chandrasekhar 1964), and therefore can be modeled more accurately than the eigenfunction. Through minimizing the difference between the modeled and observed eigenfrequencies, helioseismology validated the Sun's mean structure predicted by standard solar models (ChristensenDalsgaard et al. 1996), which gave impetus to the finding of new physics that solved the solar neutrino problem (Bahcall 2004).

The eigenfrequency of any given mode is $m$-degenerate for a spherically-symmetric Sun; all $m$ share the same frequency at given $n$ and $l$. Rotation will lift this degeneracy by causing a frequency shift dependant on $m$, and hence global mode frequencies provide a good diagnostic of the Sun's rotation profile. By using the variation of frequency with $m$, global helioseismology measured the Sun's rotation profile along the latitudinal direction at different depths, which shows a differentially rotating convection zone of roughly the same rotation rate at all depths and a uniformly rotating radiation zone (see, e.g., Schou et al. 1998, Larson and Schou 2018). Particularly, a rather counter-intuitive result revealed a faster rotating surface at the equator than at the bottom of convection zone, which implies non-trivial momentum transport processes in the solar convection zone.

Though a huge success has been achieved to measure the Sun's mean structure and rotation profile, the eigenfrequencies of solar oscillations possess very little sensitivity to meridional circulation and non-axisymmetric convective flows. In order to probe such flows, attempts have been made to use the cross-spectrum of different mode eigenfunctions (see, e.g., Woodard et al. 2013, Woodard 2016, Hanasoge 2018). These measurements, however, suffer from systematic errors that are most likely due to our inadequate understanding on the phase variation of mode eigenfunctions in the near-surface layer (Woodard et al. 2013). More modeling effort is required to resolve this issue. In order to probe these features, local helioseismology is used.

\subsubsection{Local helioseismology}

Local helioseismology refers to the various helioseismic techniques that are sensitive to the Sun's local three-dimensional structure, which all attempt an interpretation of the full wavefield of solar oscillations (see, e.g., Gizon and Birch 2005, Gizon et al.|2010). These include Fourier-Hankel decomposition, ring-diagram analysis, time-distance helioseismology, and helioseismic holography. In the following, I will briefly introduce all these techniques except for helioseismic holography, which is the main topic of this dissertation and will be discussed extensively in Section 1.3 .

The Fourier-Hankel method decomposes p-mode waves from a surface annulus into an incoming and an outgoing part using Hankel functions (Braun et al. 1987). An application of the technique to p-mode waves around sunspots shows that sunspots strongly absorb the incoming waves (Braun et al. 1987) and shift the phase between the incoming and outgoing waves (Braun|1995). 
Ring-diagram analysis is a generalization of global helioseismology that uses the power spectrum of solar oscillations from localized patches of the solar surface (Hill 1988). Specifically, the three dimensional Fourier transform is performed along two orthogonal directions $\hat{e}_{x}$ and $\hat{e}_{y}$ and time. This gives the wave power as a function of frequency and two spatial wavenumbers $k_{x}$ and $k_{y}$. For regions devoid of flows, waves possess no preferred spatial direction, therefore circular rings are formed in the two dimensional power spectrum at any given frequency. A local flow will break this symmetry, and hence distort the rings. This distortion of the rings provides a good diagnostic of the local flow structure, and is used to probe the Sun's subsurface flow fields on a daily basis (Bogart et al. 2011ab).

Time-distance helioseismology analyzes the wave travel time between any two points observed at the solar surface, which is sensitive to subsurface flows and sound-speed inhomogeneities (Duvall et al. 1993). Subsurface perturbations alter the travel times from the model, and thus contain information about the structure below the surface missing from our models. Time-distance helioseismology has been used to probe the internal structure of the Sun, such as the wavelike behavior of supergranulation (Gizon et al.2003, Langfellner et al. 2018), the structure of meridional circulation (Giles 2000, Liang et al. 2018b), subsurface flows of active regions (Braun 1997, Gizon et al. 2009), and vortical flows associated with supergranules (Langfellner et al. 2014). The biggest issue facing time-distance helioseismology is systematic errors arising as unphysical travel time shifts seen at different center-to-limb distances, which is most likely due to granulation (Schou 2015). A proper solution to this issue is yet to be discovered.

\subsection{Helioseismic holography}

Helioseismic holography aims at imaging the Sun's three-dimensional structure through focusing p-mode waves into any location of the Sun. The current framework of helioseismic holography was developed in the 1990s, when Lindsey and Braun (1990) first proposed the potential diagnostic capabilities of numerically focusing the observed $\mathrm{p}$ mode waves into the Sun's far-side/interior to study the structure therein. This idea was supported by a later study, where Braun et al. (1992) demonstrated the imaging capability of the surface wave power on subsurface acoustic anomalies of active regions. The breakthrough of helioseismic holography came in 1997, when Lindsey and Braun (1997) coined the concept of the 'egression', the back-propagation (in time) of the observed pmode waves into any depth of the Sun. This invention (of the egression) empowers, for the first time, a computational focusing of the p-mode waves into any desired location of the Sun, and opens a new window to study the Sun's internal structure. Furthermore, statistical properties of the egression were proposed to be used as solar oscillations are randomly excited (Lindsey and Braun 1997). Specifically, the variance of the egression was proposed to detect the (variance of) acoustic sources, whereas the covariance of the egression and its forward-propagating counterpart, the 'ingression', to probe scatterers such as flows and sound-speed inhomogeneities. The above formed the basis of helioseismic holography, which led to various applications that probe the Sun's far-side/interior (see introductions in Paper I and II for more detail).

The observed property that helioseismic holography interprets is the two-point covari- 
ance function of solar oscillations on the surface. In principle, the maximum amount of the Sun's structural information is retrieved of analyzing the covariance functions at all possible two-point pairs of observed waves. This is, however, a formidable task due to the large amount of data involved. For example, an image of the size $4096 \times 4096$ pixels contains of the order of $10^{14}$ different two-point pairs, which is not trivial to analyze. A constructive solution is provided by helioseismic holography where all these two-point covariance functions are utilized. Specifically, the egression and the ingression can be thought of as weighted sums of observed waves, thereby helioseismic holography analyzes a weighted sum of all two-point covariance functions at any target location. This approach is much cheaper than analyzing all the covariance functions individually, while still utilizing all of the data. Furthermore, the weighting (of observed waves) is based on physical arguments, i.e., wave propagation in the Sun, which facilitates a probe of Sun's structure at the desired location.

Despite the success achieved by helioseismic holography, we still lack an interpretation of its measurements that is mathematically rigorous and physically meaningful. Preliminary work by Skartlien (2001, 2002) revealed a measurement of the local strength of acoustic sources by the egression, based on the assumption of a deterministic source of wave excitation. Ruzmaikin and Lindsey (2003) discussed the behavior of the signal-tonoise ratio at different frequencies using the ray theory of wave propagation. Braun and Birch (2008a) measured the standard deviation of holographic travel times at different target locations. Yet, none of these studies provided a thorough theoretical explanation of the signal and noise in helioseismic holography, which is crucial for tests and design of strategies sensitive to the various flow structures in the solar interior.

One of the most important tasks of holographic techniques has been finding the optimal back propagation method, i.e., the three-dimensional back propagation of the observed wave field on a surface. The most successful achievement so far has been the Kirchhoff's integral theorem, which states that the wave field of the solution of the Helmholtz equation is uniquely determined from observations on a closed surface. Kirchhoff's integral theorem, however, is only valid when the medium is source-free. This is not the case for the Sun, where turbulent convection stochastically and ubiquitously generates solar oscillations within the interior. For waves propagating in a medium that is not sourcefree, a so-called Porter-Bojarski (PB) integral (see, e.g., Porter 1969) has been used in the research field of acoustics as the back propagation method. The application of this method has thus far not been rigorously implemented in the solar context. The PB integral and the 'egression' (the current approach) was compared by Skartlien (2002). However, no conclusion was made of the optimal back propagation that should be applied to helioseismic holography (see introduction of Paper I for more detail).

The Green's function is of great importance for back propagation methods, since it describes the impulse response of wave propagation, and hence is used to back propagate the wave field. The current Green's function applied in helioseismic holography is based on the ray theory of wave propagation, which allows for a fast computation of holograms. However, the ray theory assumes an infinitesimal wavelength of waves, which is only valid for scatterers of size bigger than the first Fresnel zone (see, e.g., Birch et al. 2001). Recent advances in computational helioseismology permit a fast and accurate computation of the Green's function that considers the finite wavelength of the wave field (see, e.g., Gizon et al. 2017). An application of such Green's functions should improve the 
current imaging capability of helioseismic holography.

\subsection{Results contained in this work}

The goal of this dissertation is to develop a mathematically rigorous and physically meaningful framework for the signal and noise in helioseismic holography, which is crucial for an accurate interpretation of holographic measurements in the Sun. Furthermore, this will facilitate tests and design on novel strategies that can improve the current imaging capability of the Sun's internal flow structure. This dissertation mainly focuses on the modeling of the PB integral, specifically, to test and validate its potential diagnostic value of the Sun's internal structure and dynamics.

Chapter 2 investigates whether the PB integral improves the current performance of holography. Both the PB hologram and the egression are mathematical representations of the Huygen's principle; they both measure the local strength of acoustic sources. A proof of concept study shows a comparison between these two methods in a homogeneous medium. The results show that the PB integral and the egression have similar spatial resolution when locating acoustic sources. However, the egression suffers from 'ghost images', artificial signals located away from the source location, whereas the PB integral does not. This means the PB integral can potentially improve the imaging capability of helioseismic holography.

Chapter 3 continues the previous work to apply the PB integral to helioseismic holography, whereas the framework is developed to investigate the signal and noise. Solar oscillations are simplified as the solution of a Helmholtz-like equation with a spherically symmetric background taken from a standard solar model, and are randomly excited by a stationary and spatially uncorrelated source function. Furthermore, scatterers such as flows and sound-speed inhomogeneties are assumed to be small perturbations on top of the reference background. In an application to the randomly excited solar oscillations, the covariance of PB integrals are used as measurements. We then use the first-order Born approximation to relate scatterers to perturbations of reference measurements. Our computation finds a resolution of half the local wavelength of holographic measurements, which validates the previous conjecture that helioseismic holography is diffraction limited. We also investigate the behavior of holographic measurements under the realization noise due to the random nature of wave excitation. We find rapid frequency variations of signal and noise at low frequencies for deep scatterers, due to contributions from individual long-lived modes of low angular degrees that can reach the deep solar interior. Additionally, the results show a very low signal-to-noise ratio of measurements above the frequency cutoff, which disagrees with the previous prediction of a low noise level at high frequencies based on the ray theory of wave propagation.

Chapter 4 attempts to find the optimal strategy in helioseismic holography to probe the Sun's internal flows. Two different flow measuring strategies are discussed and compared. The so-called lateral-vantage holography uses different pupil geometries to backward and forward (in time) propagate observed waves into any desired location of the Sun. The difference of wave travel times between the two pupils to the target location is mostly sensitive to flows near the target location, and as such has been used to probe solar subsurface flows. Another approach, hereafter the holographic correlation, propagates all observed 
waves to two different target points, whereas the difference of wave travel times from the pupil to the two target locations should be mainly susceptible to flows along the direction connected by the two target points. This approach is a direct analogy of time-distance helioseismology in three dimensions, and is expected to better probe the Sun's internal flows than lateral-vantage holography, since it utilizes all the data in each measurement. In order to validate this prediction, we compare the spatial resolution and the signal-tonoise ratio between these two flow measuring strategies using the framework developed in Chapter 3. Our computation shows that the holographic correlation has a higher spatial resolution and signal-to-noise ratio than lateral-vantage holography, thereby can improve the current imaging capability of the various subsurface flow structures in the Sun. 


\section{Paper I: Ghost images in helioseismic holography? Toy models in a uniform medium}

\subsection{Abstract}

Helioseismic holography is a powerful technique used to probe the solar interior based on estimations of the 3D wavefield. Porter-Bojarski holography, which is a well-established method used in acoustics to recover sources and scatterers in $3 \mathrm{D}$, is also an estimation of the wavefield, and hence it has the potential to be applied to helioseismology. Here we present a proof of concept study, where we compare helioseismic holography and PorterBojarski holography under the assumption that the waves propagate in a homogeneous medium. We consider the problem of locating a point source of wave excitation inside a sphere. Under these assumptions, we find that the two imaging methods have the same capability of locating the source, with the exception that helioseismic holography suffers from "ghost images" (i.e., artificial peaks away from the source location). We conclude that Porter-Bojarski holography may improve the current method used in helioseismology. ${ }^{1}$

\subsection{Introduction}

Local helioseismology is a powerful tool used to probe the 3D interior of the Sun by exploiting the information contained within the acoustic and surface-gravity waves observed at the surface (see, e.g., Gizon and Birch 2005, Gizon et al. 2010). Helioseismic holography is one branch of local helioseismology, which aims at imaging the subsurface structure by estimating the wavefield inside the Sun (Lindsey and Braun 1997, 2000a). One significant achievement of helioseismic holography has been the detection of active regions on the far-side of the Sun (far-side imaging: Lindsey and Braun 2000b). The technique used in far-side imaging, known as phase-sensitive holography, has been validated with synthetic data (see, e.g., Hartlep et al.2008, Birch et al.2011, Braun|2014), and it is extensively used in studying active regions in the near hemisphere (e.g., Braun and Birch

\footnotetext{
${ }^{1}$ This chapter reproduces the article Ghost Images in Helioseismic Holography? Toy Models in a Uniform Medium by D. Yang, published in Solar Physics 293, 17 (2018), DOI https://doi .org/10.1007/ s11207-018-1246-0. Reproduced under the terms of the Creative Commons Attribution 4.0 International License (http://creativecommons . org/licenses/by/4.0/).
} 
2008b, Braun 2016).

The fundamental concept of helioseismic holography is that the wavefield can be estimated by the so-called "egression", which is the back-propagation (in time) of the observed wavefield at the surface into the solar interior (see reviews by Lindsey and Braun 2000a, Lindsey et al. 2011). The egression can be understood in terms of Huygens' principle, whereby each point of a wavefront is considered a source, and the wavefield at a later time as a superposition of waves emitted from all of these point sources along the wavefront (see, e.g., Landau and Lifshitz 1975). Specifically, each arbitrarily small section of the observed wavefield can be regarded as a point source, and the egression as the sum of the back-propagated (in time) waves generated from all these point sources. Therefore, the egression behaves in the same manner as the wavefield propagating backward in time. Furthermore, the propagation of the wavefield forward in time is known as the "ingression". The ingression can be understood by Huygens' principle in the same way as the egression, but for the waves that are forward-propagating in time.

Since Huygens' principle is frequently used in optics and acoustics, it is thus not surprising that techniques similar to the egression/ingression have been used in fields outside helioseismology. In ocean acoustics, Jackson and Dowling (1991) proposed an active method called phase conjugation, which used the same principle as the egression, to locate acoustic sources. This method records the wavefield on an array of detectors at a fixed surface, and then it creates a time-reversed (or a complex-conjugatation in the frequency domain) wave by treating the wavefield observed at each detector as a point source. Jackson and Dowling (1991) argued that the newly created wave will focus on the source of the original wave. This proposed method was later confirmed by experiments in the sea (see, e.g., Kuperman et al. 1998, Song et al. 1998).

The rigorous mathematical statement of Huygens' principle is the Helmholtz-Kirchhoff theorem, which states that the wavefield can be reconstructed in 3D space if both the wavefield and its normal derivative are recorded on an arbitrary closed surface (see, e.g., Born and Wolf 1999). However, the Helmholtz-Kirchhoff theorem is only valid for a source-free medium (Porter and Devaney 1982). This is not the case for the entirety of the solar interior, where the wavefield is stochastically and ubiquitously excited by nearsurface convection. In this case the medium is not source-free, and to our knowledge, no theory has been established thus far that can reconstruct the wavefield in 3D space directly from observations on a 2D surface.

Although the direct reconstruction of the wavefield is not possible, acoustic sources and scatterers can be estimated in 3D space for a medium that is not source-free. In acoustics, a well-established technique known as Porter-Bojarski (PB) holography can achieve this (see, e.g., Porter and Devaney 1982, Devaney and Porter 1985, Devaney 2012). PB holography is based on an integral that is slightly different from the Helmholtz-Kirchhoff theorem; however, the wavefield and its normal derivative are still required (see Section 2.3). Instead of reconstructing the wavefield, PB holography produces a 3D image (known as the PB hologram) from a closed surface that is equivalent to the difference between the wavefield and its time-reversal (Porter 1969). Understanding the PB hologram can be achieved through Huygens' principle. Specifically, the wavefield and its normal derivative recorded at a surface can be thought of as a dipole and a monopole source, respectively. This means that the $\mathrm{PB}$ hologram is also an estimation of the wavefield, and hence it has the potential to be applied to helioseismology in a similar manner to the 
egression.

With the possible application of PB holography, it is then natural to ask whether this new method better estimates the wavefield than the egression, and hence it improves the current imaging capabilities of heliseismic holography. Previous studies by Skartlien (2001, 2002) have shown that both the egression and the PB hologram are measurements of the local strength of acoustic sources and can be related to the sources via their respective sensitivity kernels. This allows us to refine the scope of the previous question; specifically, which method is more accurate at locating acoustic sources? Comparisons between these methods have been done in ocean acoustics, where Jackson and Dowling (1991) showed that both methods can locate the source. Hence, the authors concluded that the egression is the simplified version of the PB hologram, and chose to use the egression as their preferred method since it is easier to implement. In the case of helioseismology, however, the question of the optimal method has yet to be answered. This is the goal of this article. Additionally, preliminary work by Lindsey and Braun (2004) showed that helioseismic holography suffers from unintended mirror-like images ("ghost images") due to the use of only a monopole source. An examination of ghost images in the egression and the PB hologram will also be a focus of this study.

In this article, we present a proof of concept study, where we compare the sourcesensitivity kernels of helioseismic holography and PB holography by assuming waves are propagating in a homogeneous medium. Specifically, we will examine which method is more accurate at locating acoustic sources, and hence estimating the wavefield. Additionally, we will examine the affect of observational coverage area on the kernels. This will provide an opportunity to improve the current method used in helioseismology. This article is organized as follows: Section 2.3 states the derivation of source-sensitivity kernels of helioseismic holography (egression) and PB holography, Section 2.4 states the toy model used in this paper, Section 2.5 compares the two methods with discussion and conclusions in Section 2.6 .

\subsection{Source-sensitivity kernels}

In this section, we will present how helioseismic holography and PB holography are related to acoustic sources. We will work entirely in the temporal Fourier domain using the convention

$$
f(\omega)=\int_{-\infty}^{+\infty} \mathrm{d} t F(t) e^{\mathrm{i} \omega t}
$$

where $f(\omega)$ is the Fourier transform of a given function $F(t)$.

The egression $\left[\Phi^{\mathrm{LB}}\right]$, as described by Lindsey and Braun (1997) (LB), is one of the basic quantities used in helioseismic holography,

$$
\Phi_{A}^{\mathrm{LB}}(\boldsymbol{r}, \omega)=\int_{A} \mathrm{~d}^{2} \boldsymbol{r}^{\prime} G^{*}\left(\boldsymbol{r}, \boldsymbol{r}^{\prime}, \omega\right) \Psi\left(\boldsymbol{r}^{\prime}, \omega\right),
$$

where $\boldsymbol{r}$ denotes the focal point, $A$ is the coverage of the wavefield $\Psi\left(\boldsymbol{r}^{\prime}, \omega\right)$ at any point $\boldsymbol{r}^{\prime}$ on the solar surface, and $G\left(\boldsymbol{r}, \boldsymbol{r}^{\prime}, \omega\right)$ is the Green's function associated to a wave operator defined below with the asterisk denoting the complex conjugate. For simplicity, we will drop the $\omega$ within the function's arguments for the remainder of this study. Further 
definitions and explanations concerning the Green's function will be given later in this section.

The PB hologram $\Phi^{\mathrm{PB}}$ is defined by Devaney and Porter (1985),

$$
\Phi_{A}^{\mathrm{PB}}(\boldsymbol{r})=\int_{A} \mathrm{~d}^{2} \boldsymbol{r}^{\prime}\left\{\Psi\left(\boldsymbol{r}^{\prime}\right) \partial_{n^{\prime}} \operatorname{Im} G\left(\boldsymbol{r}, \boldsymbol{r}^{\prime}\right)-\operatorname{Im} G\left(\boldsymbol{r}, \boldsymbol{r}^{\prime}\right) \partial_{n^{\prime}} \Psi\left(\boldsymbol{r}^{\prime}\right)\right\}
$$

where $\partial_{n^{\prime}}$ denotes the outward normal derivative with respect to $\boldsymbol{r}^{\prime}$, and $\operatorname{Im} G$ is the imaginary part of the Green's function.

In order to relate $\Phi^{\mathrm{LB}}$ and $\Phi^{\mathrm{PB}}$ to the acoustic sources, we first need to determine the impulse response function [ $G$, the Green's function] of the wave equation. Here we assume that the wavefield $[\Psi]$ is related to the sources through the application of a linear wave operator $[\mathcal{L}]$,

$$
\mathcal{L} \Psi(\boldsymbol{r})=S(\boldsymbol{r}),
$$

where $S(\boldsymbol{r})$ is the source function. For generality, we choose not to explicitly state $\mathcal{L}$ here. The Green's function is the impulse response of Equation 2.4, and is defined as the solution to

$$
\mathcal{L} G\left(\boldsymbol{r}, \boldsymbol{r}_{s}\right)=\delta\left(\boldsymbol{r}-\boldsymbol{r}_{s}\right),
$$

where $\delta\left(\boldsymbol{r}-\boldsymbol{r}_{s}\right)$ is the Dirac delta function and $\boldsymbol{r}_{s}$ is the location of the source. One property of the Green's function, which is crucial to deriving source sensitivity kernels, is that it can be used to solve Equation 2.4 through

$$
\Psi\left(\boldsymbol{r}^{\prime}\right)=\int_{\mathbb{R}^{3}} \mathrm{~d}^{3} \boldsymbol{r}_{s} G\left(\boldsymbol{r}^{\prime}, \boldsymbol{r}_{s}\right) S\left(\boldsymbol{r}_{s}\right) .
$$

Through expansion of $\Psi$ in Equation 2.2 with the definition in Equation 2.6, the egression becomes

$$
\Phi_{A}^{\mathrm{LB}}(\boldsymbol{r})=\int_{A} \mathrm{~d}^{2} \boldsymbol{r}^{\prime} G^{*}\left(\boldsymbol{r}, \boldsymbol{r}^{\prime}\right) \int_{\mathbb{R}^{3}} \mathrm{~d}^{3} \boldsymbol{r}_{s} G\left(\boldsymbol{r}^{\prime}, \boldsymbol{r}_{s}\right) S\left(\boldsymbol{r}_{s}\right),
$$

and through a change in the order of integration, one obtains the definition for the source sensitivity kernel $\left[K^{\mathrm{LB}}\right]$ for the egression

$$
\Phi_{A}^{\mathrm{LB}}(\boldsymbol{r})=\frac{1}{4 \pi} \int_{\mathbb{R}^{3}} \mathrm{~d}^{3} \boldsymbol{r}_{s} K_{A}^{\mathrm{LB}}\left(\boldsymbol{r}, \boldsymbol{r}_{s}\right) S\left(\boldsymbol{r}_{s}\right)
$$

where

$$
K_{A}^{\mathrm{LB}}\left(\boldsymbol{r}, \boldsymbol{r}_{s}\right)=4 \pi \int_{A} \mathrm{~d}^{2} \boldsymbol{r}^{\prime} G^{*}\left(\boldsymbol{r}, \boldsymbol{r}^{\prime}\right) G\left(\boldsymbol{r}^{\prime}, \boldsymbol{r}_{s}\right) .
$$

We note that the $4 \pi$ factor is included here such that $K^{\mathrm{LB}}$ possesses a desired nearunitary amplitude for this study.

The same procedure is repeated for the derivation of the PB hologram by expanding $\Psi$ in Equation 2.3 with Equation 2.6 and changing the order of integration;

$$
\begin{aligned}
\Phi_{A}^{\mathrm{PB}}(\boldsymbol{r}) & =\frac{1}{4 \pi} \int_{\mathbb{R}^{3}} \mathrm{~d}^{3} \boldsymbol{r}_{s} K_{A}^{\mathrm{PB}}\left(\boldsymbol{r}, \boldsymbol{r}_{s}\right) S\left(\boldsymbol{r}_{s}\right), \\
K_{A}^{\mathrm{PB}}\left(\boldsymbol{r}, \boldsymbol{r}_{s}\right) & =4 \pi \int_{A} \mathrm{~d}^{2} \boldsymbol{r}^{\prime}\left\{G\left(\boldsymbol{r}^{\prime}, \boldsymbol{r}_{s}\right) \partial_{n^{\prime}} \operatorname{Im} G\left(\boldsymbol{r}, \boldsymbol{r}^{\prime}\right)-\operatorname{Im} G\left(\boldsymbol{r}, \boldsymbol{r}^{\prime}\right) \partial_{n^{\prime}} G\left(\boldsymbol{r}^{\prime}, \boldsymbol{r}_{s}\right)\right\},
\end{aligned}
$$


where $K_{A}^{\mathrm{PB}}$ is the source sensitivity kernel of the PB hologram.

A comparison of the egression and the PB hologram requires only the knowledge of their respective source-sensitivity kernels, whereas details of the source function are not needed. Therefore, we will examine the source-sensitivity kernels of the two imaging methods in this study. We will compare the two source kernels under simplifying assumptions about the medium in which the waves propagate. We note that, in practice, the egression power $\left|\Phi^{\mathrm{LB}}\right|^{2}$ is used to estimate the location of acoustic sources, since the wavefield in the Sun is stochastically excited (see, e.g., Lindsey and Braun 1997, Hanson et al. 2015). Therefore, we will also compare the squared modulus of the source kernels.

\subsection{Toy model: Waves in a homogeneous medium}

With Equations 2.9 and 2.11 in hand, we require the computation of the Green's functions in order to determine the source-sensitivity kernels. In general, a Green's function can be obtained numerically for any given linear operator $[\mathcal{L}]$. However, as stated in the introduction, we examine a homogeneous medium and as such the Green's function can be computed analytically.

We consider this homogeneous medium in $\mathbb{R}^{3}$ space with a constant sound speed $c=$ $10^{5} \mathrm{~m} \cdot \mathrm{s}^{-1}$, and we adopt the linear wave operator,

$$
\mathcal{L}=k^{2}+\nabla^{2}, k=\frac{\omega+\mathrm{i} \gamma}{c},
$$

where $k$ is the wavenumber, $\omega$ is the angular frequency and $\gamma$ is the damping rate. The solution of Equation 2.5 with the above wave operator and free boundary condition is given by

$$
G\left(\boldsymbol{r}, \boldsymbol{r}^{\prime}\right)=-\frac{1}{4 \pi} \frac{\exp \left(\mathrm{i} k\left|\boldsymbol{r}-\boldsymbol{r}^{\prime}\right|\right)}{\left|\boldsymbol{r}-\boldsymbol{r}^{\prime}\right|},
$$

which is also known as the outgoing free-space Green's function (Born and Wolf 1999). In this study, we set the damping rate $[\gamma]$ to be $0.1 \%$ of the angular frequency, and we use a Cartesian coordinate system $(x, y, z)$ with its origin at the center of a sphere $\mathrm{V}_{\odot}$ with the radius $\mathrm{R}_{\odot}=696 \mathrm{Mm}$.

Current observational capabilities mean that we can only observe the wavefield on a fraction of the solar surface. To study the consequence of this limitation on observations, we will examine both the case where the entire surface is observed and the case where only a fraction of the solar surface is observed. In these cases, we assume the sources are located along the $z$-axis, the coverage is symmetric with respect to the $z$-axis and is centered above the North Pole $\left(0,0, \mathrm{R}_{\odot}\right)$. Under these assumptions, $K^{\mathrm{LB}}$ and $K^{\mathrm{PB}}$ are axisymmetric about the $z$-axis.

\subsection{Results}

\subsubsection{Source-sensitivity kernels at $3 \mathrm{mHz}$}

Here we examine the source-sensitivity kernels $\left[K^{\mathrm{LB}}\right.$ and $\left.K^{\mathrm{PB}}\right]$ at a frequency of $\omega / 2 \pi=$ $3 \mathrm{mHz}$. Figure 2.1 shows $2 \mathrm{D}$ slices of both the real and imaginary parts of $K^{\mathrm{LB}}$ and 


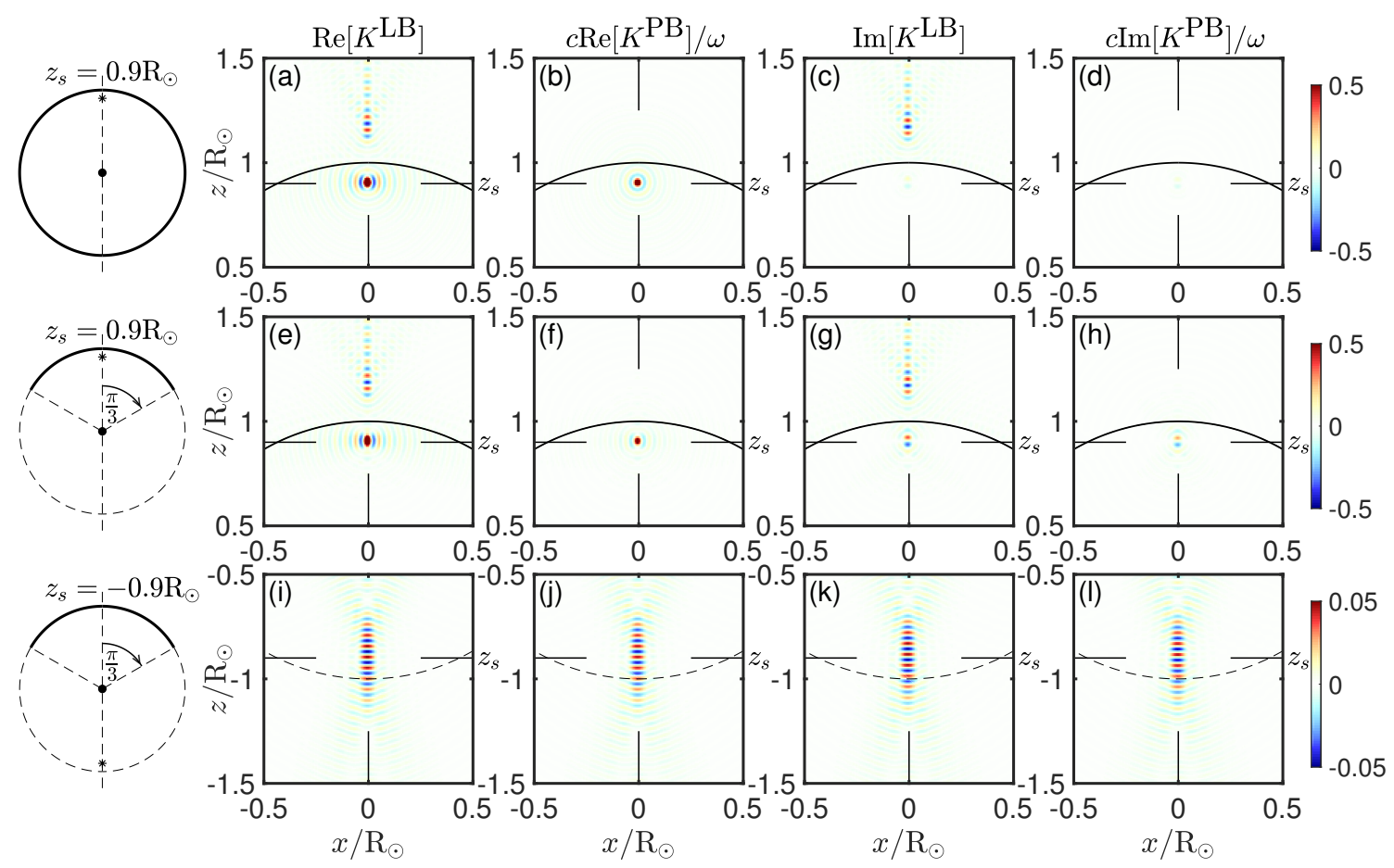

Figure 2.1: 2D slices of the real and imaginary parts of $K^{\mathrm{LB}}$ and $K^{\mathrm{PB}}$ through the $z$-axis, when the entire surface (top row) and 60 degrees around the North Pole (middle and bottom rows) can be observed. The location of the source is at the focal point of the cross hairs (solid black lines) in each plot, being $z_{s}=0.9 \mathrm{R}_{\odot}$ in the top and middle rows and $z_{s}=-0.9 \mathrm{R}_{\odot}$ in the bottom row. A simple geometry plot is given on the left of each row, with the source location (asterisk), the solar center (big dot), solar surface (dashed lines), and coverage area (solid arc) on top of it.

$c K^{\mathrm{PB}} / \omega$ through the $z$-axis, for the source locations at $z_{s}=0.9 \mathrm{R}_{\odot}$ (panel a to $\mathrm{h}$ ) and $-0.9 \mathrm{R}_{\odot}$ (panel $\mathrm{i}$ to $\mathrm{l}$ ) on the $z$ axis. The factor $c / \omega$ is added to $K^{\mathrm{PB}}$ so that the kernel is dimensionless like the egression. The first row of panels show the source-sensitivity kernels under the assumption that the entire surface is observed. The remaining rows show the kernels assuming a coverage of 60 degrees from the North Pole. Considering all panels, both $\operatorname{Re}\left[K^{\mathrm{LB}}\right]$ and $\operatorname{Re}\left[K^{\mathrm{PB}}\right]$ peak at the source, while in comparison $\operatorname{Im}\left[K^{\mathrm{LB}}\right]$ and $\operatorname{Im}\left[K^{\mathrm{PB}}\right]$ are negligible if the source is located at the near side. These results demonstrate that both $\operatorname{Re}\left[K^{\mathrm{LB}}\right]$ and $\operatorname{Re}\left[K^{\mathrm{PB}}\right]$ can locate the source in both of these coverage geometries. In the case of far-side located sources, all of the kernels have became less localized. While these kernels can locate the sources, we also see that the egression kernels (both the real and imaginary parts) have "ghost images" above the surface, while the PB holograms do not. These ghost images appear as peaks at points away from the source location. We note that in this work we also observed ghost images below the surface in the egression, when the source is above the surface. This suggests that the egression cannot distinguish sources from below and above the surface, since one can not differentiate sources and ghosts. The PB holograms do not suffer from this problem. Further explanations and discussions concerning the ghost images will be given in Section 2.6.1.

For a more focused comparison of the kernels in Figure 2.1, Figure 2.2 shows 1D 

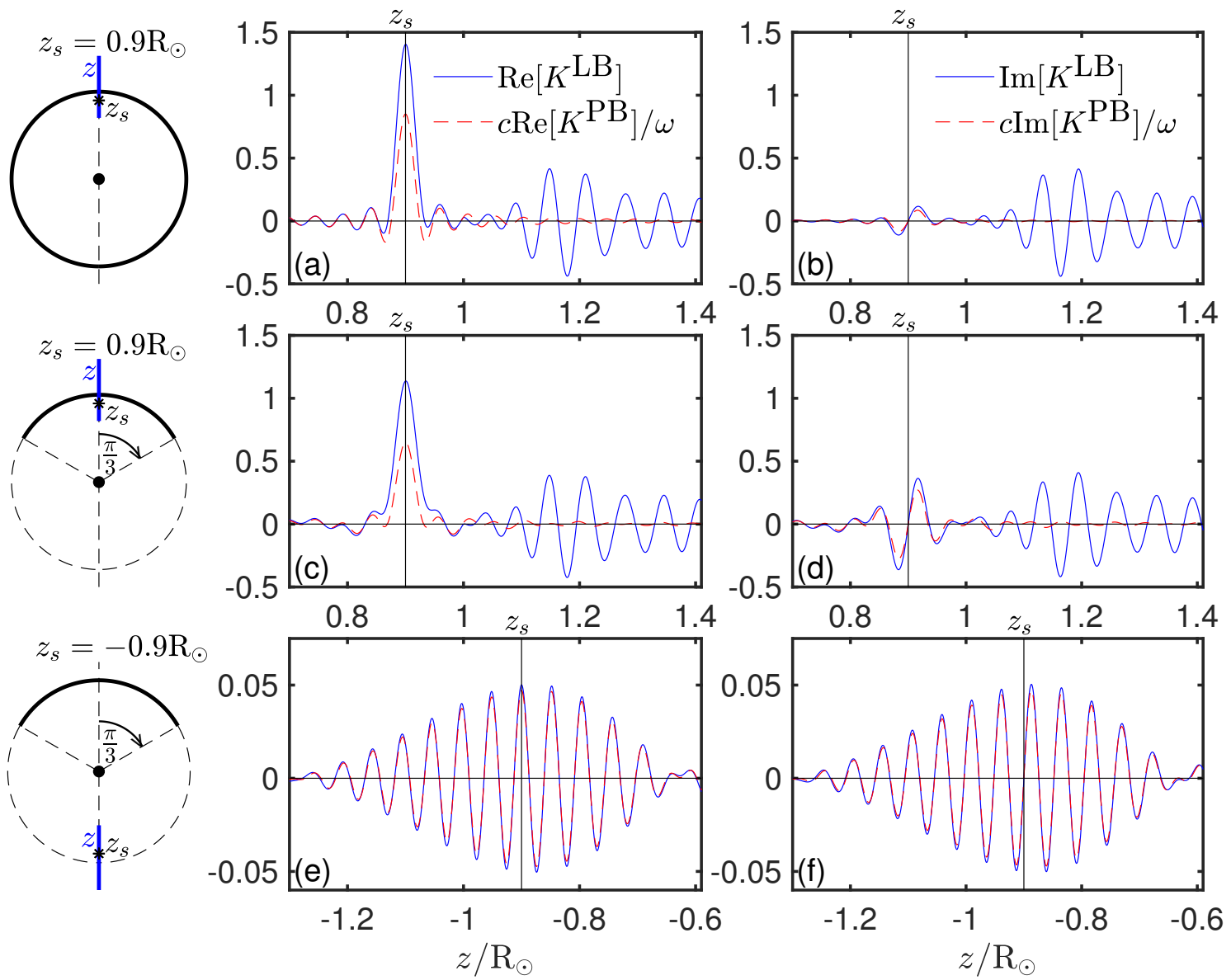

Figure 2.2: $1 \mathrm{D}$ slices of $K^{\mathrm{LB}}$ (blue solid) and $K^{\mathrm{PB}}$ (red dashed) shown in Figure 2.1 along the $z$ axis. The real parts are plotted in panels a, c, and e, and the imaginary parts in panels $\mathrm{b}, \mathrm{d}$, and $\mathrm{f}$. The geometry is shown on the left with the addition of the slice length (blue line).

slices of $K^{\mathrm{LB}}$ and $K^{\mathrm{PB}}$ along the $z$-axis with the real parts shown in panel a, c, and e, and the imaginary parts in panel $\mathrm{b}, \mathrm{d}$, and $\mathrm{f} . \operatorname{Re}\left[K^{\mathrm{LB}}\right]$ and $\operatorname{Re}\left[K^{\mathrm{PB}}\right]$ again have peaks at the source location. Here we see that despite the coverage geometry, $\operatorname{Im}\left[K^{\mathrm{LB}}\right]$ and $\operatorname{Im}\left[K^{\mathrm{PB}}\right]$ are always zero at the source location with the peaks seen in Figure 2.1 surrounding the source location. This suggests that $\operatorname{Im}\left[K^{\mathrm{LB}}\right]$ and $\operatorname{Im}\left[K^{\mathrm{PB}}\right]$ cannot pinpoint the exact source location. In the case of far-side located sources, $K^{\mathrm{LB}}$ and $K^{\mathrm{PB}}$ are both highly oscillatory and non-localized, and hence recovering the source location may be problematic with observations at a single frequency.

Figure 2.3 shows $1 \mathrm{D}$ slices of $K^{\mathrm{LB}}$ and $K^{\mathrm{PB}}$ along a line that is perpendicular to the $z$-axis and passes through the source. Due to axial symmetry, only half of the slice is plotted. Here the imaginary parts of $K^{\mathrm{LB}}$ and $K^{\mathrm{PB}}$ are not shown since they are negligible compared to the real parts. Unlike the vertical slices, both $K^{\mathrm{LB}}$ and $K^{\mathrm{PB}}$ do not have ghost images, and they are less oscillatory when the source is located at the far-side. 

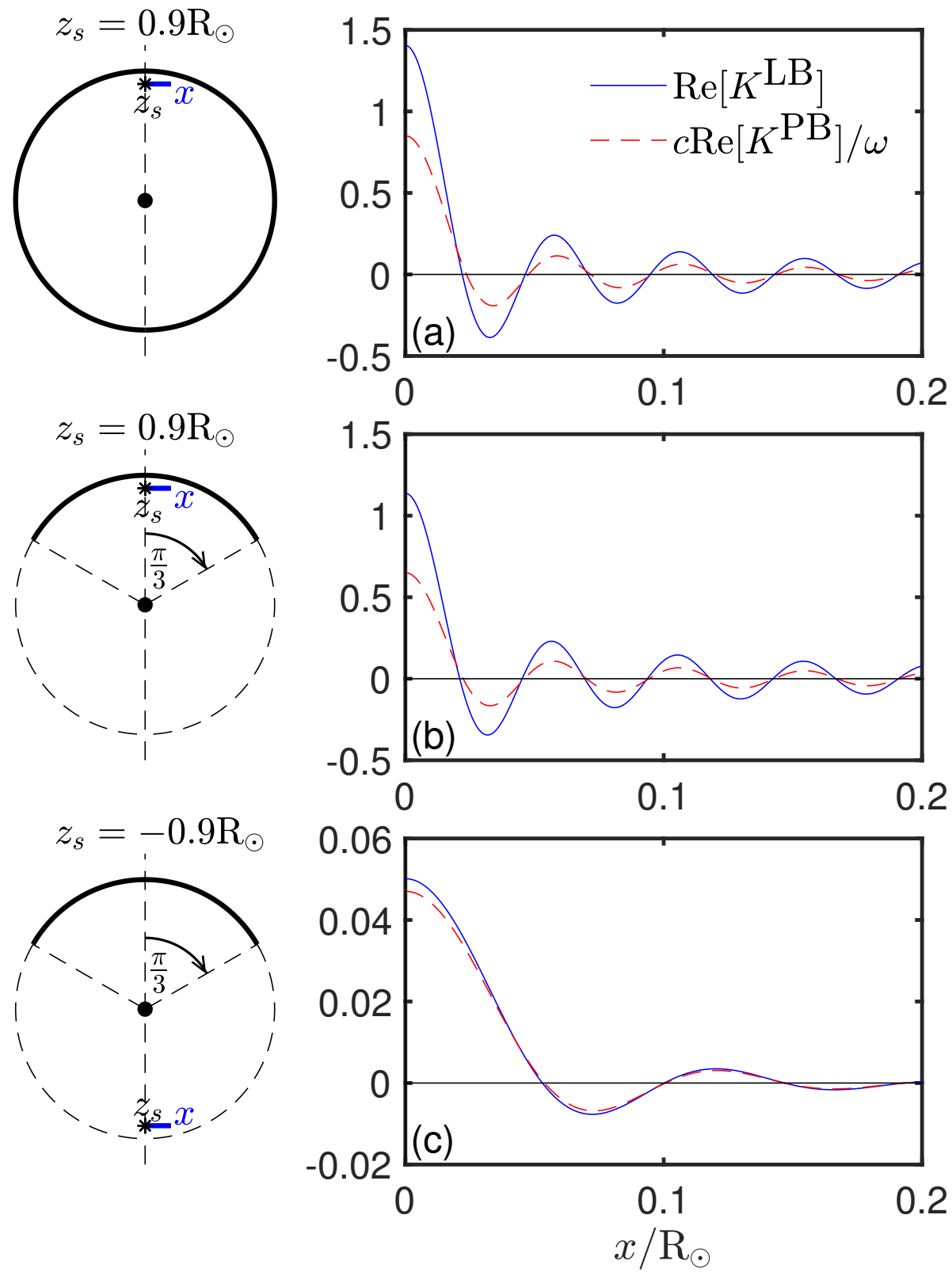

Figure 2.3: $1 \mathrm{D}$ slices of $K^{\mathrm{LB}}$ (blue solid) and $K^{\mathrm{PB}}$ (red dashed) in Figure 2.1 along the line perpendicular to the $z$-axis. The imaginary parts are negligible compared to the real parts, and they are not shown in the figure. Due to axial symmetry, only half of the slice is shown. The geometry of the slices is shown on the left with the slice length shown in blue.

\subsubsection{Kernels averaged over frequency}

Specifically, for observations at a single frequency both methods are highly oscillatory and non-localized for the far-side located source, and the egression suffers from ghost images for the near-side located source. One possible solution to the above issues is to average kernels over a number of frequencies, since the ghost images for the near-side 
located source and the side-lobes for the far-side located source may peak at different locations for different frequencies.
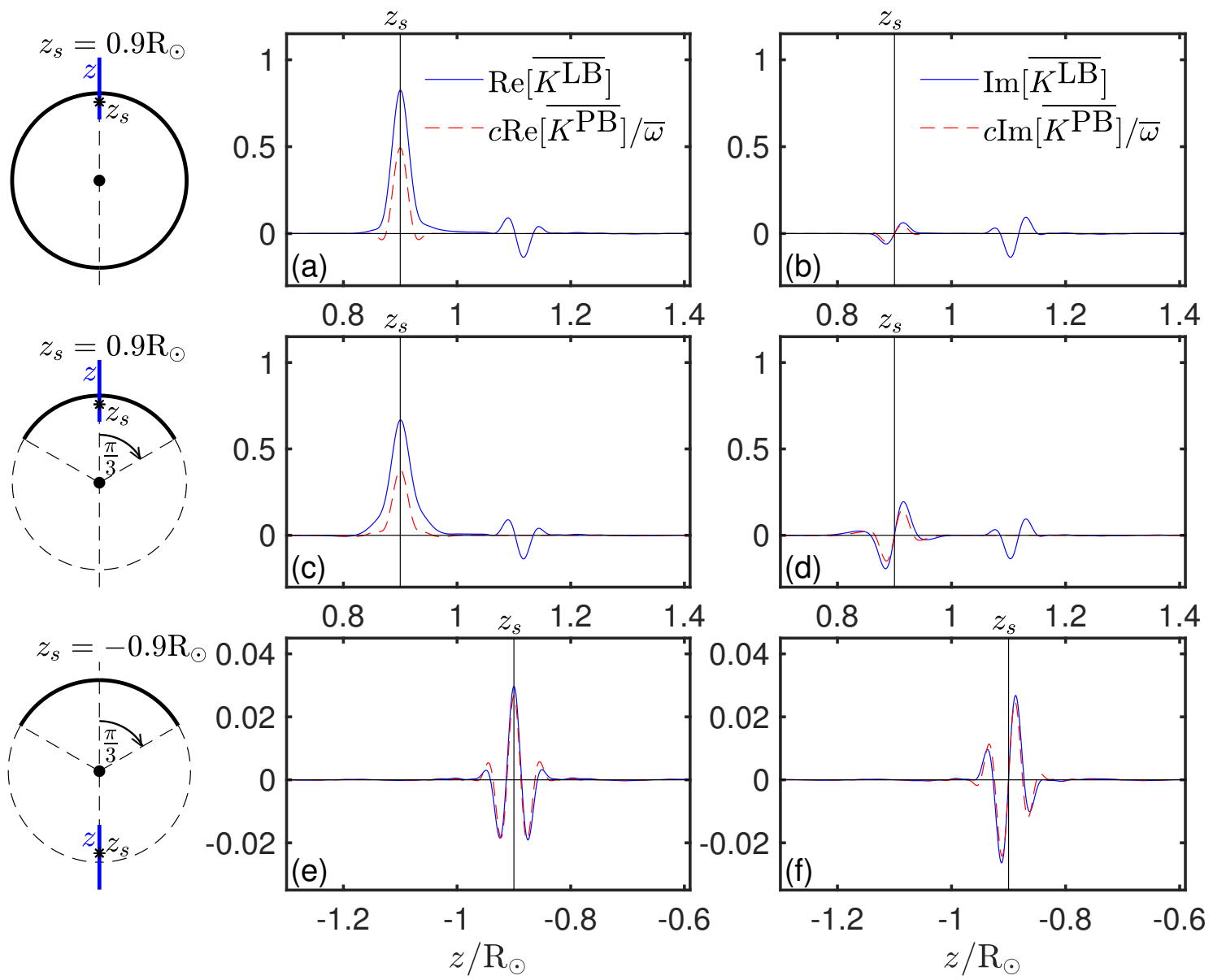

Figure 2.4: 1D slices of kernels averaged over frequencies using a Gaussian weight function centered at $3 \mathrm{mHz}$ with a standard deviation of $1 \mathrm{mHz}$. Here we use a bar to denote the averaged quantity. The observational coverage $A$ is depicted in the left panels (thick arcs). The frequency averaging reduces the amplitudes of the ghost images for the nearsided source and the side-lobes for the source on the far-side.

Figure 2.4 shows $1 \mathrm{D}$ vertical slices of $K^{\mathrm{LB}}$ and $K^{\mathrm{PB}}$ averaged from 41 frequencies equally distributed from 1 to $5 \mathrm{mHz}$. A Gaussian weight function centered at $3 \mathrm{mHz}$ and with a standard deviation of $1 \mathrm{mHz}$ had been applied for the averaging. From these results, it is clear that averaging kernels over frequency reduces the amplitude of the ghost images for the near-sided source and the side-lobes for the far-side located source. The averaged kernels along the horizontal direction are similar to the kernels with a single frequency, and as such they are not shown here.

As mentioned in Section 2.3, the egression power, which is related to the source covariance via $\left|K^{\mathrm{LB}}\right|^{2}$, has been used in observations as estimations of the acoustic sources. Therefore, the effect of averaging $\left|K^{\mathrm{LB}}\right|^{2}$ and $\left|K^{\mathrm{PB}}\right|^{2}$ over different frequencies is also of great interest. Figure 2.5 shows a comparison of $\left|K^{\mathrm{LB}}\right|^{2}$ and $\left|K^{\mathrm{PB}}\right|^{2}$ with or without averaging over different frequencies. Only the slices along the vertical direction are shown, 

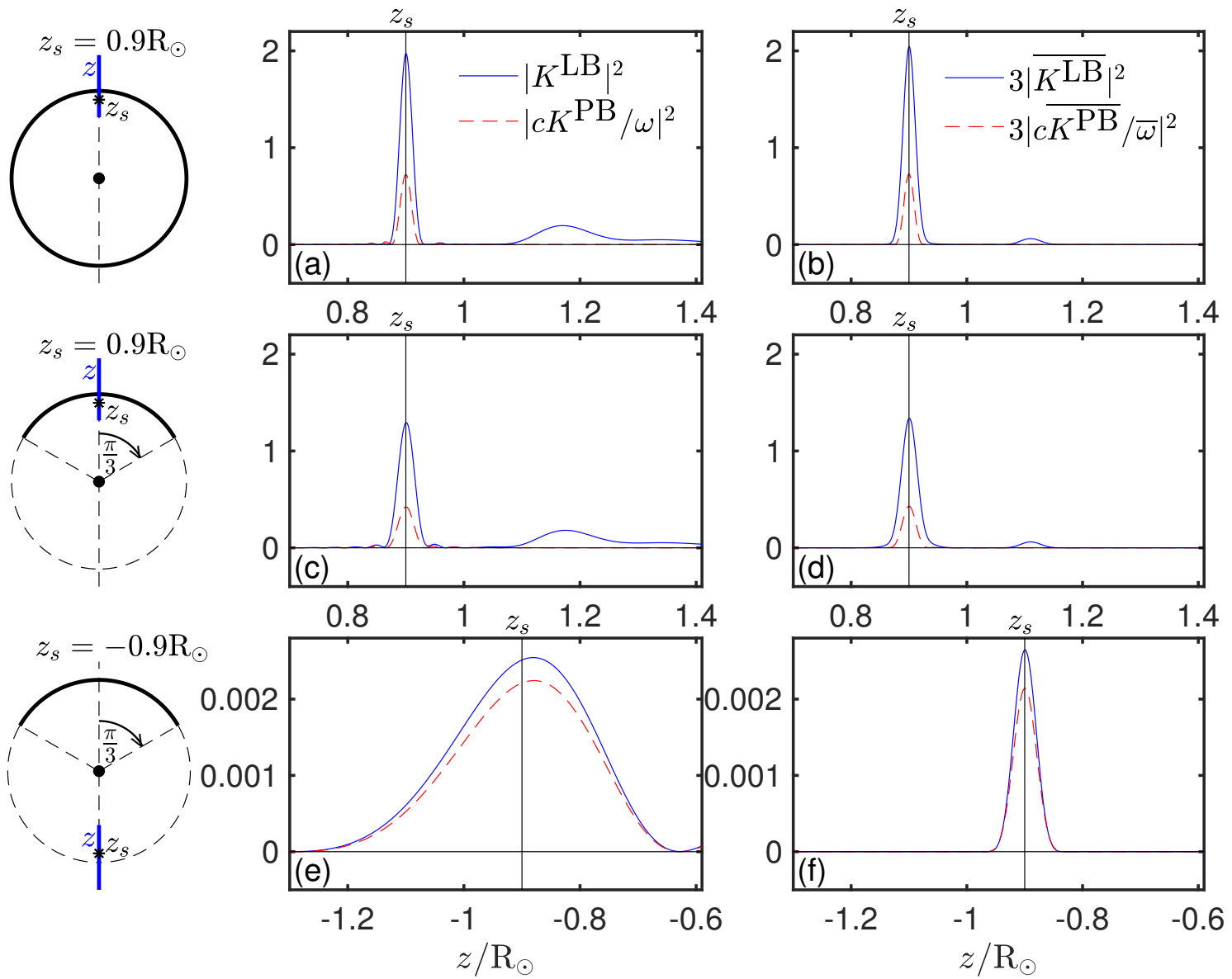

Figure 2.5: $1 \mathrm{D}$ slices of $\left|K^{\mathrm{LB}}\right|^{2}$ and $\left|K^{\mathrm{PB}}\right|^{2}$ in a plane containing the $z$-axis. The left panels are for a single frequency of $3 \mathrm{mHz}$. The right panels are for averages over frequencies using a Gaussian weight function centered at $3 \mathrm{mHz}$ with a standard deviation of $1 \mathrm{mHz}$. We see that averaging the kernels over frequencies reduces the amplitude of the ghosts when the source is located on the near-side, and it improves the spatial resolution when the source is located on the far-side.

as the difference between $\left|K^{\mathrm{LB}}\right|^{2}\left(\left|K^{\mathrm{PB}}\right|^{2}\right)$ from a single frequency at $3 \mathrm{mHz}$ and averaged from 1 to $5 \mathrm{mHz}$ along the horizontal direction is small. In the vertical direction, averaging $\left|K^{\mathrm{LB}}\right|^{2}$ and $\left|K^{\mathrm{PB}}\right|^{2}$ over different frequencies reduces the amplitude of the ghosts when the source is located on the near-side, and improves the spatial resolution when the source is located on the far-side.

\subsubsection{Dependence of the spatial resolution on the coverage}

The results thus far have shown that both of the methods can locate the source, though the egression has the complication of ghost peaks. The question then arises of how well do these methods resolve the sources with differing observational coverages? We define the spatial resolution the egression and the $\mathrm{PB}$ hologram as the full width at the half maximum (FWHM) of $\left|K^{\mathrm{LB}}\right|^{2}$ and $\left|K^{\mathrm{PB}}\right|^{2}$ respectively. From Figures 2.2 and 2.3 we can see that both of the methods behave differently in the vertical and horizontal directions, and as such the 
FWHM in these two directions are considered separately. Additionally, to quantify the affect of averaging kernels over different frequencies, both the FWHM for kernels from a single frequency at $3 \mathrm{mHz}$ and the value averaged over frequencies from 1 to $5 \mathrm{mHz}$ are considered.

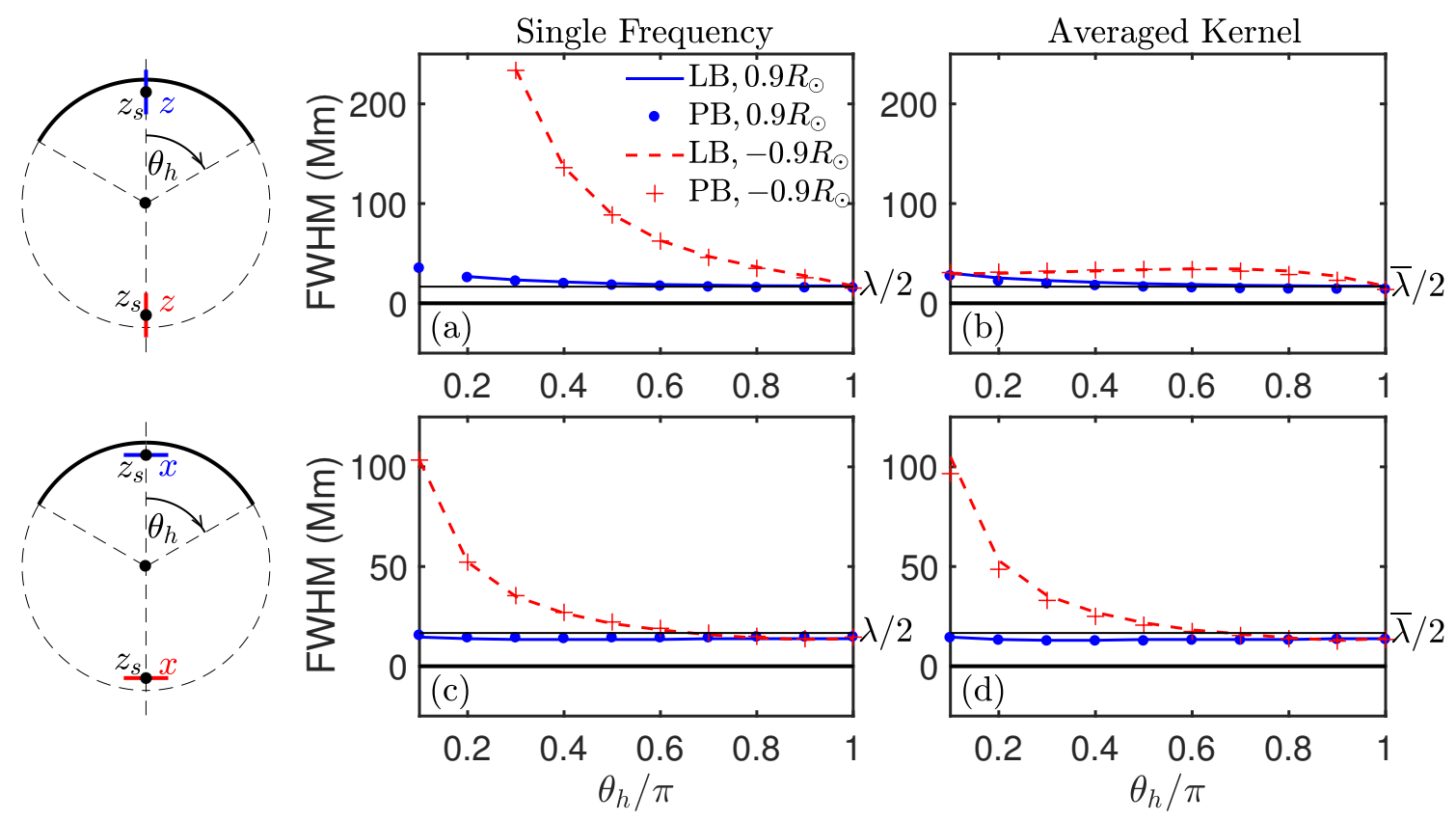

Figure 2.6: The full width at half maximum (FWHM) of $\left|K^{\mathrm{LB}}\right|^{2}$ and $\left|K^{\mathrm{PB}}\right|^{2}$ as a function of the angle $\left[\theta_{h}\right]$, which defines the observational coverage (cap of area $A$ ). The FWHM along two directions, horizontal and vertical, are shown in the case of a near-side located source $\left(z_{s}=0.9 \mathrm{R}_{\odot}\right.$, blue $)$ and a far-side located source $\left(z_{s}=-0.9 \mathrm{R}_{\odot}\right.$, red $)$. The theoretical resolution limit of $\lambda / 2$ is also shown with a horizontal black line. Additionally, both the results for the kernels at a single frequency $\omega / 2 \pi=3 \mathrm{mHz}$ and the frequency-averaged kernels are shown on the left and right columns, respectively.

Figure 2.6 shows the FWHM of $\left|K^{\mathrm{LB}}\right|^{2}$ and $\left|K^{\mathrm{PB}}\right|^{2}$ as a function of the angle $\left[\theta_{h}\right]$, which defines the observational coverage (cap of area $[A]$ ). The FWHM along vertical (top row) and horizontal (bottom row) directions are considered in the case of kernels at $3 \mathrm{mHz}$ (left column) and averaged from 1 to $5 \mathrm{mHz}$ (averaged kernel, right column). At $3 \mathrm{mHz}$, the difference between the two imaging methods is small, which implies that either method has the same capability to resolve the source. Furthermore, the FWHM is close to the resolution limit despite the size of the coverage area when the source is located at the near-side $\left(z_{s}=0.9 \mathrm{R}_{\odot}\right)$. When the source is located at the far-side $\left(z_{s}=-0.9 \mathrm{R}_{\odot}\right)$, the resolution improves (FWHM decreases) with increasing coverage. When averaging over different frequencies, a clear improvement of the spatial resolution can be found along the vertical direction when the source is located on the far-side, while the spatial resolution is almost the same as before for other cases. 


\subsection{Discussion}

\subsubsection{Ghost images in the egression}
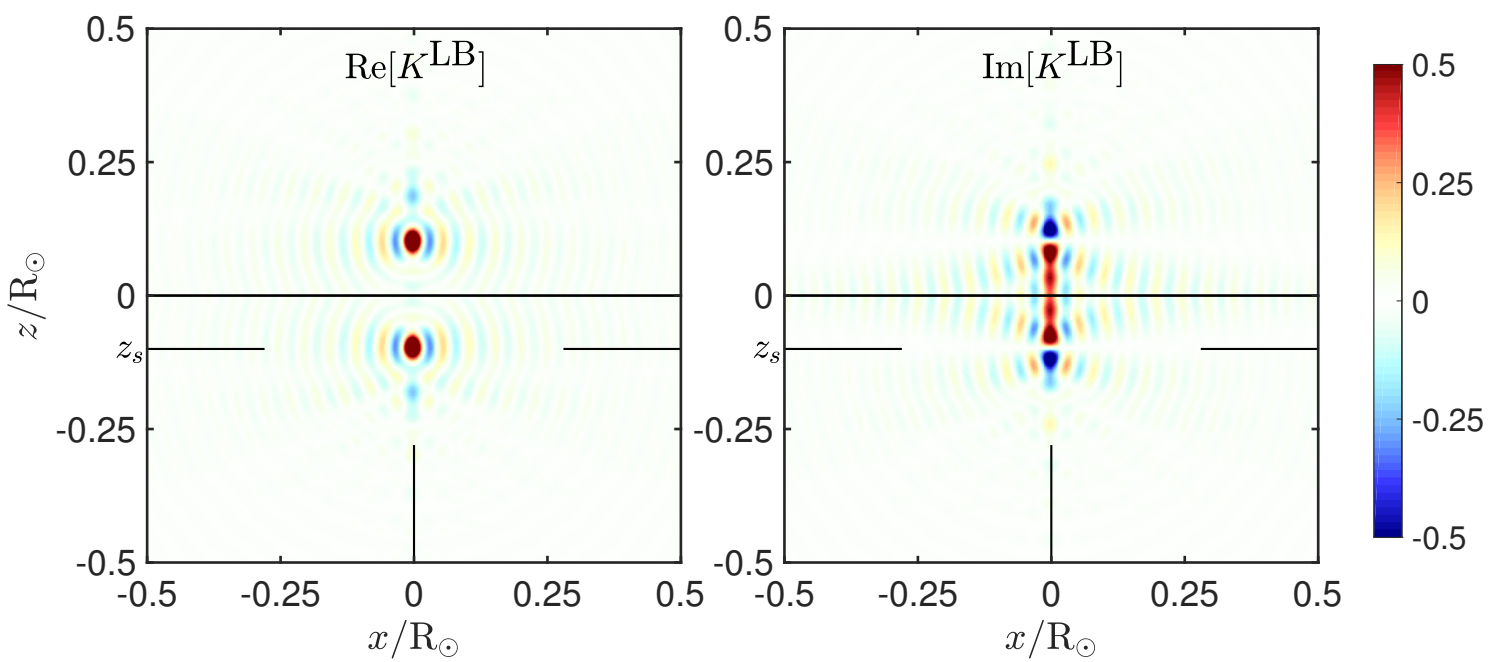

Figure 2.7: 2D slice of the real (left panel) and imaginary (right panel) part of $K^{\mathrm{LB}}$ through the $z$-axis when the wavefield is observed at the $z=0$ plane. Here the coverage is a circle that centered at the origin and with the radius of $\mathrm{R}_{\odot}$. The source is located along the $z$-axis at $z_{s}=-0.1 \mathrm{R}_{\odot}$ and is indicated by the focus of the cross hairs in each plot.

The appearance of ghost images in the egression can be understood by Huygens' principle, whereby each arbitrarily small section of the observed wavefield is regarded as a point source, and the egression as a superposition of the back-propagated (in time) waves generated from all the point sources. Furthermore, each newly created wave is spherically symmetric with respect to its source location in a homogeneous medium, and thus it will propagate in all directions with the same behavior. This is the cause of the ghost images. A clear example of this is when the wavefield is recorded on a plane, where all of the newly created waves are symmetric with respect to the recording surface, and hence the egression will focus on both the source location and its counterpart on the other side of the surface (see Figure 2.7 for an example). When the wavefield is observed on a sphere, however, the newly created waves are no longer symmetric with respect to the surface, and the ghost images show a complicated diffraction pattern due to the interference among the newly created waves (Figure 2.1). This provides a simple explanation for the ghost images seen in the egression above the surface (see also Lindsey and Braun 2004).

The PB hologram does not suffer from ghost images like the egression, since it includes not only a monopole source but also a dipole source, which is not symmetric with respect to the source location. Additionally, the amplitudes of the monopole and dipole sources are chosen such that the PB hologram only focuses on the source location.

Future work should include a solar-like density stratification to confirm this simple explanation. The sharp drop in density at the solar surface leads to a reflection of the waves below $5.3 \mathrm{mHz}$, which is not captured in our toy model. 
We also note that Lindsey and Braun (2005a b) proposed that ghost images may explain the presence of phase anomalies observed around active regions in phase-sensitive holography. For further implications and discussions about the ghost images in helioseismic holography, we refer readers to Lindsey and Braun (2004).

\subsubsection{The ingression and the PB hologram}

In this study, we have not considered the ingression in our analysis. The ingression is an equally important quantity used in helioseismic holography, which is an estimation of where the wavefield converges to by propagating the wavefield forward in time (Lindsey and Braun 1997). So far, we have considered the wavefield $\Psi$ as diverging away from the source. However, to compare the ingression and the PB hologram, a wavefield that converges from infinity to the source location is desired. Such a wavefield can be achieved by considering the wavefield diverging from the source as before, but with the reversed sign in time, i.e., $\Psi(\boldsymbol{r},-t)$. In the frequency domain, this time-reversal corresponds to taking the complex conjugate. Additionally, the wave number $[k]$ in the wave equation is also conjugated and thus the wavefield decays when propagating towards the source location (Devaney 2012). In this case, the ingression is

$$
\Phi_{A,-}^{\mathrm{LB}}(\boldsymbol{r}, \omega)=\int_{A} \mathrm{~d}^{2} \boldsymbol{r}^{\prime} G\left(\boldsymbol{r}, \boldsymbol{r}^{\prime}, \omega\right) \Psi^{*}\left(\boldsymbol{r}^{\prime}, \omega\right),
$$

and the PB hologram becomes

$$
\Phi_{A,-}^{\mathrm{PB}}(\boldsymbol{r}, \omega)=\int_{A} \mathrm{~d}^{2} \boldsymbol{r}^{\prime}\left\{\Psi^{*}\left(\boldsymbol{r}^{\prime}, \omega\right) \partial_{n^{\prime}} \operatorname{Im} G\left(\boldsymbol{r}, \boldsymbol{r}^{\prime}, \omega\right)-\operatorname{Im} G\left(\boldsymbol{r}, \boldsymbol{r}^{\prime}, \omega\right) \partial_{n^{\prime}} \Psi^{*}\left(\boldsymbol{r}^{\prime}, \omega\right)\right\} .
$$

We can see that $\Phi_{-}^{\mathrm{LB}}$ and $\Phi_{-}^{\mathrm{PB}}$ are simply the complex conjugates of $\Phi^{\mathrm{LB}}$ and $\Phi^{\mathrm{PB}}$. Since we have discussed the real and imaginary parts and the power of $\Phi^{\mathrm{PB}}$ and $\Phi^{\mathrm{LB}}$ separately in the results, those of $\Phi^{\mathrm{LB}}\left(\Phi^{\mathrm{PB}}\right)$ will be the same as $\Phi_{-}^{\mathrm{LB}}\left(\Phi_{-}^{\mathrm{PB}}\right)$. In particular, $\Phi_{-}^{\mathrm{LB}}$ will also have ghost images while $\Phi_{-}^{\mathrm{PB}}$ will not, and $\left|\Phi_{-}^{\mathrm{LB}}\right|^{2}$ and $\left|\Phi_{-}^{\mathrm{PB}}\right|^{2}$ will have the same spatial resolution when imaging acoustic sources.

\subsubsection{Application to stereoscopic helioseismology}

Results in Section 2.5.3 showed that the spatial resolution of the hologram on the far-side increases as the coverage area increases. Thus the resolution has a fundamental limit when observing from a single vantage point. It has been suggested to combine observations from two or several vantage points to increase the observation coverage and therefore to improve the spatial resolution (and signal-to-noise ratio) of holography. Stereoscopic helioseismology is believed to be our best chance to probe the subsurface structure in the polar regions and the deep convection zone, which is crucial for understanding the 11year solar cycle (see, e.g., Ruzmaikin and Lindsey 2003). This conjecture, however, has not been studied in detail.

Figure 2.8 shows the PB hologram at the solar surface with a Dirac delta source located $0.7 \mathrm{Mm}$ below the surface at $270^{\circ}$ longitude along the Equator. Different coverage geometries are considered in the case of a single spacecraft (top row), two spacecraft in 

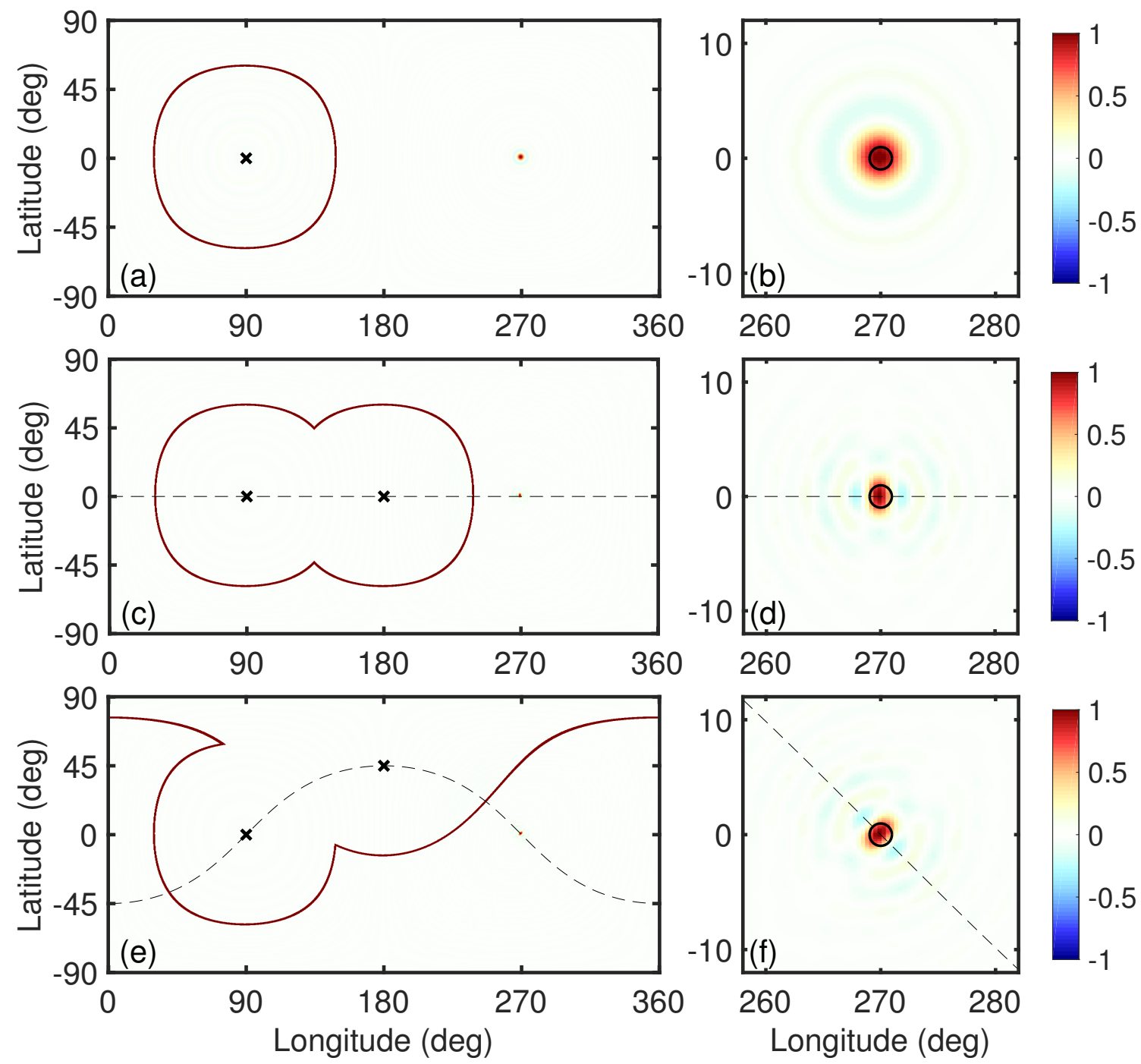

Figure 2.8: $2 \mathrm{D}$ slices of $\operatorname{Re}\left[K^{\mathrm{PB}}\right]$ at the solar surface for observations from a single spacecraft (top row), two spacecraft in the Ecliptic (middle row), and two spacecraft with one in the Ecliptic and the other at $45^{\circ}$ inclination (bottom row). Here the source is located $0.7 \mathrm{Mm}$ below the surface at $270^{\circ}$ longitude along the Equator, and the plots are shown after divided by the maximum value of $\operatorname{Re}\left[K^{\mathrm{PB}}\right]$. We note that $\operatorname{Im}\left[K^{\mathrm{PB}}\right]$ is negligible, and as such is not shown here. We plot $\operatorname{Re}\left[K^{\mathrm{PB}}\right]$ at the entire surface on the left column, where the boundary of the coverage is marked by a red curve and the point below the spacecraft by a cross. Additionally, a zoom of images around the source location are shown on the right column, where a circle centered above the source location and with a diameter of the wavelength is added on each plot. We can see a clear improvement of the spatial resolution when a second spacecraft is added. Furthermore, a preferred direction that possesses higher spatial resolution is found along the great circle (black-dashed line) that goes through the points below the two spacecraft.

the ecliptic (middle row), and two spacecraft with one in the Ecliptic and the other at $45^{\circ}$ inclination (bottom row). Here only the real parts of the PB hologram are shown, since 
the imaginary parts are negligible. The results show a clear improvement of the spatial resolution when a second spacecraft is added, whereas the FWHM along the Equator is about two times smaller than that of a single spacecraft. Additionally, the spatial resolution is increased along the great circle (black-dashed line) at the intersection of the plane that contains the center of the sphere and the two spacecraft.

Stereoscopic helioseismology might be implemented in future space missions such as Solar Orbiter and Solar Activity Far Side Investigation (see, e.g., Sekii et al.2015 and references therein) together with observations collected from the ground (Global Oscillation Network Group) or from near-Earth orbit (Solar Dynamics Observatory). In particular, the Polarimetric and Helioseismic Imager onboard Solar Orbiter is to be launched soon and will provide high-resolution line-of-sight velocity and continuum intensity at the photosphere, which are suitable for helioseismic studies (Woch and Gizon|2007, Müller et al. 2013, Löptien et al. 2015). The orbit of Solar Orbiter will have a period of 168 days during the nominal mission and reach a heliographic latitude of up to $25^{\circ}$ (35 during an extended mission) (Müller et al. 2013). This means that Solar Orbiter will cover a large range of spacecraft-Sun-Earth angles to test stereoscopic helioseismology.

\subsection{Outlook}

In this article we found that helioseismic holography and PB holography are similar techniques, with the exception that the egression and the ingression suffer from ghost images. In principle, we could apply the PB holograms to phase-sensitive holography by replacing the egression and the ingression with the appropriate $\Phi^{\mathrm{PB}}$ and $\Phi_{-}^{\mathrm{PB}}$. Our toy model suggests that the PB holograms will improve current helioseismic holography since they do not suffer from ghost images. However additional modeling work is needed. Future studies should consider random acoustic sources and scatterers. Furthermore, the computations must be carried out in a solar-like stratified background medium. Finally, in order to implement PB holography a method for determining the normal derivative of the wavefield needs to be developed.

\section{Acknowledgements}

I thank Laurent Gizon, Aaron C. Birch, Damien Fourier, and Chris S. Hanson for their useful insights and comments on the article. I also thank the referee, Charles Lindsey, for his thoughtful comments. This work was supported by the International Max Planck Research School (IMPRS) for Solar System Science at the University of Göttingen. 



\section{Paper II: Signal and noise in helioseismic holography}

\subsection{Abstract}

Helioseismic holography is an imaging technique used to study heterogeneities and flows in the solar interior from observations of solar oscillations at the surface. Holographic images contain noise due to the stochastic nature of solar oscillations. We provide a theoretical framework for modeling signal and noise in Porter-Bojarski helioseismic holography. The wave equation may be recast into a Helmholtz-like equation, so as to connect with the acoustics literature and define the holography Green's function in a meaningful way. Sources of wave excitation are assumed to be stationary, horizontally homogeneous, and spatially uncorrelated. Using the first Born approximation we calculate holographic images in the presence of perturbations in sound-speed, density, flows, and source covariance, as well as the noise level as a function of position. This work is a direct extension of the methods used in time-distance helioseismology to model signal and noise. To illustrate the theory, we compute the holographic image intensity numerically for a buried sound-speed perturbation at different depths in the solar interior. The reference Green's function is obtained for a spherically-symmetric solar model using a finite-element solver in the frequency domain. Below the pupil area on the surface, we find that the spatial resolution of the holographic image intensity is very close to half the local wavelength. For a sound-speed perturbation of size comparable to the local spatial resolution, the signalto-noise ratio is approximately constant with depth. Averaging the image intensity over a number $N$ of frequencies above $3 \mathrm{mHz}$ increases the signal-to-noise ratio by a factor nearly equal to the square root of $N$. This may not be the case at lower frequencies, where large variations in the holographic signal are due to the individual contributions of the long-lived modes of oscillation $\sqrt[1]{1}$

\subsection{Introduction}

Local helioseismology aims at studying the solar interior in three dimensions by exploiting the information contained in the waves observed at the solar surface (e.g., Gizon

${ }^{1}$ This chapter reproduces the article Signal and noise in helioseismic holography by Laurent Gizon, Damien Fournier, Dan Yang, Aaron C. Birch, and Hélène Barucq, Astronomy \& Astrophysics 620, 136 (2018), DOI 10.1051/0004-6361/201833825. Reproduced with permission from Astronomy \& Astrophysics, (C) ESO. 
and Birch 2005, and references therein). Helioseismic holography is one particular approach of local helioseismology, which images subsurface scatterers by back-propagating the surface wave field to target points in the interior. Helioseismic holography is also known as Lindsey-Braun (LB) holography (Lindsey and Braun 1997, 2000a, and references therein). It has been used to study solar convection (Braun et al. 2004, 2007), active region emergence (Birch et al. 2013, 2016), sunspot subsurface structure (Braun and Birch 2008b, Birch et al. 2009), to image wave sources (Lindsey et al. 2006), to study sunquakes caused by solar flares (Zharkov et al. 2013, Besliu-Ionescu et al.2017), and to detect active regions on the far side of the Sun (Lindsey and Braun 2000b, Liewer et al. 2014).

In acoustics, a well-established version of holography in a medium that contains sources is Porter-Bojarski (PB) holography (Porter and Devaney 1982). PB holography uses both the wave field and its normal derivative at the surface to produce holographic images (Porter 1969). PB holography was introduced in helioseismology by Skartlien (2001, 2002), where deterministic sources and scatterers were recovered in a solar background. Yang (2018) recently studied PB holographic images in a homogeneous medium permeated by localized deterministic sources to study ghost images near the observational boundary.

In this paper we apply PB holography in a realistic helioseismological setting. First we rewrite the wave equation in Helmholtz form, in order to properly define the Green's functions that are involved in the definition of PB holographic data. The background density and sound-speed are taken from a standard solar model. The model of wave excitation is described by a reasonable source covariance function, which leads to a solarlike power spectrum for acoustic oscillations.

The signal is defined as the expectation value of the holographic image intensity that results from perturbations in sound speed, density, and flows with respect to the reference solar model. The corresponding sensitivity kernels are computed in the first-order Born approximation (Gizon and Birch 2002, Birch and Gizon 2007, Braun et al. 2007, Birch et al. 2011). This signal must take into account the correlations between incident and scattered wave fields, which are both connected to the sources of excitation (turbulent convection).

Random noise in holographic images is due to the stochastic nature of the sources of excitation. While noise can sometimes be estimated from the data (Lindsey and Braun 1990, Braun and Birch 2008a), a theoretical understanding is useful to design holographic experiments. Here we extend to holography the noise model developed in time-distance helioseismology (Gizon and Birch 2004, Fournier et al. 2014). We do not attempt to image individual sources as in Skartlien (2002), which in our view is not a well-posed problem (see also Lindsey et al.2006), except in case of imaging the sources of sunquake waves. Instead we consider sources to be specified through a source covariance function.

\subsection{Reduced wave equation}

At angular frequency $\omega$ and spatial position $r$ in the computational domain $V$, the propagation of acoustic waves in a 3D heterogeneous moving medium is described by the 
displacement vector $\boldsymbol{\xi}(\boldsymbol{r}, \omega)$, solution to

$$
-(\omega+\mathrm{i} \gamma+\mathrm{i} \boldsymbol{u} \cdot \nabla)^{2} \boldsymbol{\xi}-\frac{1}{\rho} \nabla\left(\rho c^{2} \nabla \cdot \boldsymbol{\xi}\right)+\text { gravity terms }=\boldsymbol{F},
$$

where $\rho(\boldsymbol{r})$ and $c(\boldsymbol{r})$ are the density and sound speed, and $\boldsymbol{u}(\boldsymbol{r})$ is a steady vector flow. Wave attenuation is included through the function $\gamma(\boldsymbol{r}, \omega)$. The source term $\boldsymbol{F}(\boldsymbol{r}, \omega)$ is a realization from a random process; it describes the stochastic excitation of the waves by turbulent convection. Following Lamb (1909) and Deubner and Gough (1984), we consider the scalar variable

$$
\psi=\rho^{1 / 2} c^{2} \nabla \cdot \boldsymbol{\xi}
$$

to recast the wave equation into a Helmholtz-like equation

$$
L \psi:=-\left(\Delta+k^{2}\right) \psi-\frac{2 \mathrm{i} \omega}{\rho^{1 / 2} c} \rho \boldsymbol{u} \cdot \nabla\left(\frac{\psi}{\rho^{1 / 2} c}\right)=S,
$$

where $S=\rho^{1 / 2} c^{2} \nabla \cdot \boldsymbol{F}$ is a scalar source term. The local wavenumber $k(\boldsymbol{r}, \omega)$ is given by

$$
k^{2}=\frac{\left(\omega^{2}+2 \mathrm{i} \omega \gamma\right)-\omega_{\mathrm{c}}^{2}}{c^{2}}
$$

where the squared acoustic cut-off frequency is

$$
\omega_{\mathrm{c}}^{2}=\rho^{1 / 2} c^{2} \Delta\left(\rho^{-1 / 2}\right)
$$

In obtaining Eq. (4.2), we ignored gravity terms and assumed slow variations of $c, \boldsymbol{u}$, and $\gamma$ compared to the wavelength (Gizon et al.2017). The advection term is such that the corresponding operator is Hermitian symmetric for the inner product $\left\langle\psi_{1}, \psi_{2}\right\rangle=\int \psi_{1}^{*} \psi_{2} d V$ under the conditions that the flow conserves mass and that it does not cross the boundary $\left(u_{n}=0\right.$ on $\left.\partial V\right)$.

The stochastic sources of excitation are assumed to be stationary and spatially uncorrelated. They are described by a source covariance function of the form

$$
\mathbb{E}\left[S^{*}(\boldsymbol{r}, \omega) S\left(\boldsymbol{r}^{\prime}, \omega\right)\right]=M(\boldsymbol{r}, \omega) \delta\left(\boldsymbol{r}-\boldsymbol{r}^{\prime}\right)
$$

To solve Eq. 4.2, , one needs to specify a boundary condition at the computational boundary. Here we apply an outgoing radiation boundary condition

$$
\partial_{n} \psi=\mathrm{i} k_{n} \psi \quad \text { on } \partial V .
$$

We apply the boundary condition (Atmo RBC 1) from Barucq et al. (2018), which assumes an exponential decay of the background density at the boundary of the domain but neglects curvature. Then, the local wavenumber $k_{n}$ from Eq. (3.7) is given by

$$
k_{n}^{2}=\frac{\omega^{2}+2 \mathrm{i} \omega \gamma}{c^{2}}-\frac{1}{4 H^{2}},
$$

where $H=-1 /(\mathrm{d} \ln \rho / \mathrm{d} r)$ is the density scale height at the boundary. The last term in Eq. (3.8) is connected to the cut-off frequency for an isothermal atmosphere (Lamb 1909), thus $k_{n}$ is an approximation of the wavenumber $k$ from Eq. (4.3). Fournier et al. (2017) discusses several of the boundary conditions derived in Barucq et al. (2018). 
Table 3.1: Possible wave propagators.

\begin{tabular}{ccc}
\hline \hline Wave propagator & $H_{\alpha}$ & References \\
\hline Backward & $G_{0}^{*}$ & Porter and Devaney $(1982)$ \\
Backward & $G_{0}^{-}$ & Tsang et al. (1987) \\
Backward & $\operatorname{Im} G_{0}$ & Devaney and Porter (1985) \\
Forward & $\left(G_{0}^{-}\right)^{*}$ & This work \\
\hline
\end{tabular}

\subsection{Holographic image intensity}

The following calculations are done at constant $\omega$, thus we drop $\omega$ from the list of function arguments when not explicitly needed. The Porter-Bojarski integral is defined by Porter and Devaney (1982):

$$
\Phi_{\alpha}(\boldsymbol{x}, A):=\int_{A}\left[\psi(\boldsymbol{r}) \partial_{n} H_{\alpha}(\boldsymbol{r}, \boldsymbol{x})-H_{\alpha}(\boldsymbol{r}, \boldsymbol{x}) \partial_{n} \psi(\boldsymbol{r})\right] \mathrm{d} \boldsymbol{r},
$$

where $H_{\alpha}$ is an acoustic wave propagator for the reference medium and $A$ is a surface on the Sun where $\psi$ and $\partial_{n} \psi$ are observed. The role of $H_{\alpha}$ is to propagate the wave field backward (or forward) in time, which leads to the concept of egression (or ingression) in LB holography (Lindsey and Braun 2000a).

Several choices have been proposed in the literature for the propagators, as detailed in Table 3.1. These depend on the outgoing $\left(G_{0}\right)$ and incoming $\left(G_{0}^{-}\right)$Green's functions defined in a reference medium with $\rho_{0}, c_{0}, \gamma_{0}$, and $\boldsymbol{u}_{0}=0$ :

$$
\begin{aligned}
& L_{0}\left[G_{0}\left(\boldsymbol{r}, \boldsymbol{r}^{\prime}\right)\right]=\delta\left(\boldsymbol{r}-\boldsymbol{r}^{\prime}\right) \quad \text { and } \quad \partial_{n} G_{0}=\mathrm{i} k_{n} G_{0} \text { on } \partial V, \\
& L_{0}\left[G_{0}^{-}\left(\boldsymbol{r}, \boldsymbol{r}^{\prime}\right)\right]=\delta\left(\boldsymbol{r}-\boldsymbol{r}^{\prime}\right) \quad \text { and } \quad \partial_{n} G_{0}^{-}=-\mathrm{i} k_{n} G_{0}^{-} \text {on } \partial V,
\end{aligned}
$$

with

$$
L_{0}=-\left(\Delta+k_{0}^{2}\right)
$$

where $k_{0}$ is $k$ in the reference medium and $k_{n}$ is from Eq. (3.8). The Green's functions $G_{0}^{*}$ or $G_{0}^{-}$are backward propagators (c.f. egression), while $\left(G_{0}^{-}\right)^{*}$ is a forward propagator (c.f. ingression). When the surface $A$ is closed, it is equivalent to use $G_{0}^{*}$ and $\operatorname{Im} G_{0}$ (Devaney and Porter 1985). Tsang et al. (1987) proposed $H_{\alpha}=G_{0}^{-}$as a backward propagator to correct for wave attenuation.

If the observations are made at the computational boundary and the wave field satisfies the same boundary condition as the Green's function, then Eq. (4.1) reads

$$
\Phi_{\alpha}(\boldsymbol{x}, A)=\int_{A} \psi(\boldsymbol{r})\left[\partial_{n} H_{\alpha}(\boldsymbol{r}, \boldsymbol{x})-\mathrm{i} k_{n} H_{\alpha}(\boldsymbol{r}, \boldsymbol{x})\right] \mathrm{d} \boldsymbol{r} .
$$

When $H_{\alpha}=G_{0}^{*}$, we have

$$
\Phi(\boldsymbol{x}, A)=-2 \mathrm{iRe}\left[k_{n}\right] \int_{A} \psi(\boldsymbol{r}) G_{0}^{*}(\boldsymbol{r}, \boldsymbol{x}) \mathrm{d} \boldsymbol{r}
$$


Table 3.2: Proposed propagators and associated pupils $\left(H_{\alpha}, A\right)$ and $\left(H_{\beta}, A^{\prime}\right)$ for different types of perturbations. The pupil geometries are shown in Fig. 3.1 .

\begin{tabular}{ccccc}
\hline \hline Perturbations & $H_{\alpha}$ & $A$ & $H_{\beta}$ & $A^{\prime}$ \\
\hline Source covariance & $\operatorname{Im} G_{0}$ & $P$ & $\operatorname{Im} G_{0}$ & $P$ \\
Sound speed & $\left(G_{0}^{-}\right)^{*}$ & $P$ & $G_{0}^{-}$ & $P$ \\
Flow $u_{\theta}$ & $\left(G_{0}^{-}\right)^{*}$ & $Q_{\text {south }}$ & $G_{0}^{-}$ & $Q_{\text {north }}$ \\
Flow $u_{\phi}$ & $\left(G_{0}^{-}\right)^{*}$ & $Q_{\text {east }}$ & $G_{0}^{-}$ & $Q_{\text {west }}$ \\
\hline
\end{tabular}
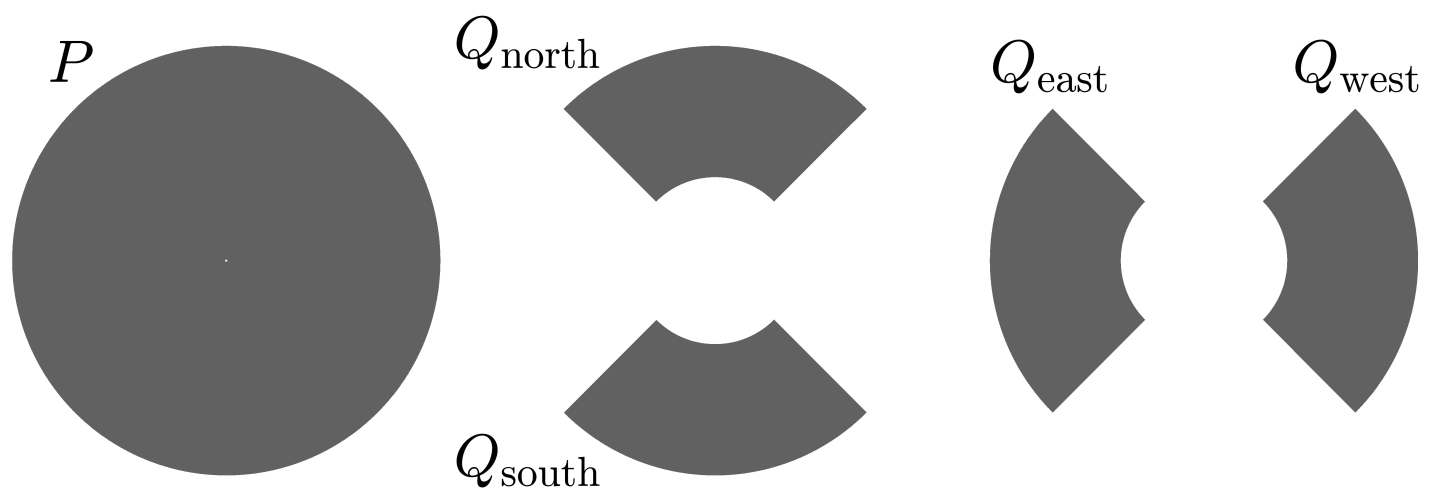

Figure 3.1: Pupil geometries used to compute sound-speed or source kernels $(P)$ and flow kernels $(Q \mathrm{~s})$, also see Table 3.2 .

which corresponds to the egression as defined by Lindsey and Braun (2000a). Thus LB and PB integrals are closely related, at least for the upper boundary condition employed here.

In LB holography, information is extracted from the egression-ingression correlation (wave-speed perturbations and flows) and from the egression power (source covariance). Analogously, we define the PB image intensity (or covariance) as

$$
I_{\alpha \beta}\left(\boldsymbol{x}, A, A^{\prime}\right)=\Phi_{\alpha}^{*}(\boldsymbol{x}, A) \Phi_{\beta}\left(\boldsymbol{x}, A^{\prime}\right) .
$$

For the case $\alpha=\beta$ we define

$$
I_{\alpha}(\boldsymbol{x}, A)=\left|\Phi_{\alpha}^{*}(\boldsymbol{x}, A)\right|^{2} .
$$

Different choices of pupils and propagators will provide sensitivity to different quantities as shown in Table 3.2. Scatterers are detected by correlating the forward and backward propagated wavefields. Different pupil shapes will give access to different components of the flow (Table 3.2 and Fig. 3.1). 


\subsection{First-order perturbations with respect to a reference solar model}

We wish to study how perturbations to the background medium affect the holographic images. Using the Born approximation, the first step is to express the perturbations to the wavefield and use this expression in Eq. (4.1) to obtain the perturbations to the PB integral and the image intensity.

\subsubsection{Perturbations to the wavefield}

We consider perturbations $\delta c, \delta \rho, \delta \gamma, \boldsymbol{u}$ with respect to the reference medium defined by $\rho_{0}, c_{0}, \gamma_{0}$, and $\boldsymbol{u}_{0}=0$. The perturbations to the sources of excitation are described through the perturbations to the source covariance,

$$
M(\boldsymbol{r}, \omega)=M_{0}(\boldsymbol{r}, \omega)+\delta M(\boldsymbol{r}, \omega)
$$

where

$$
\delta M(\boldsymbol{r}, \omega)=\mathbb{E}\left[S_{0}^{*}(\boldsymbol{r}) \delta S\left(\boldsymbol{r}^{\prime}\right)+\delta S^{*}(\boldsymbol{r}) S_{0}\left(\boldsymbol{r}^{\prime}\right)\right]=\epsilon(\boldsymbol{r}) M_{0}(\boldsymbol{r}, \omega) .
$$

Using the Born approximation up to first order, we write the wave field as

$$
\psi(\boldsymbol{r}, \omega)=\psi_{0}(\boldsymbol{r}, \omega)+\delta \psi(\boldsymbol{r}, \omega),
$$

where the zeroth- and first-order wave fields are given by

$$
\begin{aligned}
L_{0}\left[\psi_{0}\right] & =S_{0}, \\
L_{0}[\delta \psi] & =-\delta L\left[\psi_{0}\right]+\delta S .
\end{aligned}
$$

The perturbed wave operator is

$$
\delta L\left[\psi_{0}\right]=-\delta k^{2} \psi_{0}-\frac{2 \mathrm{i} \omega}{\rho_{0}^{1 / 2} c_{0}} \rho_{0} \boldsymbol{u} \cdot \nabla\left(\frac{\psi_{0}}{\rho_{0}^{1 / 2} c_{0}}\right),
$$

with

$$
c_{0}^{2} \delta k^{2}=2 \mathrm{i} \omega \delta \gamma-\left(\omega^{2}+2 \mathrm{i} \omega \gamma_{0}\right) \frac{\delta c^{2}}{c_{0}^{2}}-\left(\frac{\partial \omega_{\mathrm{c}}^{2}}{\partial \rho}\right)_{0} \delta \rho .
$$

In terms of the Green's function $G_{0}$, we have

$$
\begin{aligned}
& \psi(\boldsymbol{r})=\psi_{0}(\boldsymbol{r})+\int_{V} G_{0}\left(\boldsymbol{r}, \boldsymbol{r}_{\mathrm{s}}\right) \delta k^{2}\left(\boldsymbol{r}_{\mathrm{s}}\right) \psi_{0}\left(\boldsymbol{r}_{\mathrm{s}}\right) \mathrm{d} \boldsymbol{r}_{\mathrm{s}}+\int_{V} G_{0}(\boldsymbol{r}, \boldsymbol{s}) \delta S(\boldsymbol{s}) \mathrm{d} \boldsymbol{s} \\
& +2 \mathrm{i} \omega \int_{V} \rho_{0}\left(\boldsymbol{r}_{\mathrm{s}}\right) \boldsymbol{u}_{0}\left(\boldsymbol{r}_{\mathrm{s}}\right) \cdot \nabla\left(\frac{G_{0}\left(\boldsymbol{r}, \boldsymbol{r}_{\mathrm{s}}\right)}{\rho_{0}^{1 / 2}\left(\boldsymbol{r}_{\mathrm{s}}\right) c_{0}\left(\boldsymbol{r}_{\mathrm{s}}\right)}\right) \frac{\psi_{0}\left(\boldsymbol{r}_{\mathrm{s}}\right)}{\rho_{0}^{1 / 2}\left(\boldsymbol{r}_{\mathrm{s}}\right) c_{0}\left(\boldsymbol{r}_{\mathrm{s}}\right)} \mathrm{d} \boldsymbol{r}_{\mathrm{s}} .
\end{aligned}
$$

where the scattering location $\boldsymbol{r}_{\mathrm{s}}$ spans the entire volume $V$. 


\subsubsection{Perturbations to the PB integral}

For convenience, we introduce the source kernels

$$
K_{\alpha}(\boldsymbol{x}, \boldsymbol{s}, A)=\int_{A}\left[G(\boldsymbol{r}, \boldsymbol{s}) \partial_{n} H_{\alpha}(\boldsymbol{r}, \boldsymbol{x})-H_{\alpha}(\boldsymbol{r}, \boldsymbol{x}) \partial_{n} G(\boldsymbol{r}, \boldsymbol{s})\right] \mathrm{d} \boldsymbol{r},
$$

such that the PB integral is given by

$$
\Phi_{\alpha}(\boldsymbol{x}, A)=\int_{V} K_{\alpha}(\boldsymbol{x}, \boldsymbol{s}, A) S(\boldsymbol{s}) \mathrm{d} \boldsymbol{s} .
$$

We denote by $K_{\alpha, 0}$ the source kernel in the reference medium (when $G$ is replaced by $G_{0}$ ).

Replacing the wavefield by its first order expansion (Eq. (3.24)) in the definition of the PB integral (Eq. (4.1)), one obtains

$$
\Phi_{\alpha}(\boldsymbol{x}, A)=\Phi_{\alpha, 0}(\boldsymbol{x}, A)+\delta \Phi_{\alpha}(\boldsymbol{x}, A),
$$

where $\Phi_{\alpha, 0}$ is the value for the reference medium and $\delta \Phi_{\alpha}$ is due to perturbations in the medium:

$$
\delta \Phi_{\alpha}(\boldsymbol{x}, A)=\int_{V} \delta K_{\alpha}(\boldsymbol{x}, \boldsymbol{s}, A) S(\boldsymbol{s}) \mathrm{d} \boldsymbol{s}+\int_{V} K_{\alpha, 0}(\boldsymbol{x}, \boldsymbol{s}, A) \delta S(\boldsymbol{s}) \mathrm{d} \boldsymbol{s}
$$

where

$$
\begin{aligned}
& \delta K_{\alpha}(\boldsymbol{x}, \boldsymbol{s}, A)=\int_{V} K_{\alpha, 0}\left(\boldsymbol{x}, \boldsymbol{r}_{\mathrm{s}}, A\right) \delta k^{2}\left(\boldsymbol{r}_{\mathrm{s}}\right) G_{0}\left(\boldsymbol{r}_{\mathrm{s}}, \boldsymbol{s}\right) \mathrm{d} \boldsymbol{r}_{\mathrm{s}} \\
& \quad+2 \mathrm{i} \omega \int_{V} \frac{K_{\alpha, 0}\left(\boldsymbol{x}, \boldsymbol{r}_{\mathrm{s}}, A\right)}{\rho_{0}^{1 / 2}\left(\boldsymbol{r}_{\mathrm{s}}\right) c_{0}\left(\boldsymbol{r}_{\mathrm{s}}\right)} \rho_{0} \boldsymbol{u}_{0} \cdot \nabla\left(\frac{G_{0}\left(\boldsymbol{r}_{\mathrm{s}}, \boldsymbol{s}\right)}{\rho_{0}^{1 / 2}\left(\boldsymbol{r}_{\mathrm{s}}\right) c_{0}\left(\boldsymbol{r}_{\mathrm{s}}\right)}\right) \mathrm{d} \boldsymbol{r}_{\mathrm{s}} .
\end{aligned}
$$

\subsubsection{Perturbations to the image intensity}

To first order, we write the holographic image intensity in the form

$$
I_{\alpha \beta}\left(\boldsymbol{x}, A, A^{\prime}\right)=I_{\alpha \beta, 0}\left(\boldsymbol{x}, A, A^{\prime}\right)+\delta I_{\alpha \beta}\left(\boldsymbol{x}, A, A^{\prime}\right) .
$$

The expectation values of the zeroth- and first-order image intensities are

$$
\begin{aligned}
\mathbb{E}\left[I_{\alpha \beta, 0}\left(\boldsymbol{x}, A, A^{\prime}\right)\right]= & \int_{V} K_{\alpha, 0}^{*}(\boldsymbol{x}, \boldsymbol{s}, A) K_{\beta, 0}\left(\boldsymbol{x}, \boldsymbol{s}, A^{\prime}\right) M_{0}(\boldsymbol{s}) \mathrm{d} \boldsymbol{s}, \\
\mathbb{E}\left[\delta I_{\alpha \beta}\left(\boldsymbol{x}, A, A^{\prime}\right)\right]= & \int_{V} K_{\alpha, 0}^{*}(\boldsymbol{x}, \boldsymbol{s}, A) \delta K_{\beta}\left(\boldsymbol{x}, \boldsymbol{s}, A^{\prime}\right) M_{0}(\boldsymbol{s}) \mathrm{d} \boldsymbol{s} \\
& +\int_{V} \delta K_{\alpha}^{*}(\boldsymbol{x}, \boldsymbol{s}, A) K_{\beta, 0}\left(\boldsymbol{x}, \boldsymbol{s}, A^{\prime}\right) M_{0}(\boldsymbol{s}) \mathrm{d} \boldsymbol{s} \\
& +\int_{V} K_{\alpha, 0}^{*}(\boldsymbol{x}, \boldsymbol{s}, A) K_{\beta, 0}\left(\boldsymbol{x}, \boldsymbol{s}, A^{\prime}\right) \epsilon(\boldsymbol{s}) M_{0}(\boldsymbol{s}) \mathrm{d} \boldsymbol{s} .
\end{aligned}
$$

Using the definition of the source kernels, we obtain

$$
\mathbb{E}\left[I_{\alpha \beta, 0}\left(\boldsymbol{x}, A, A^{\prime}\right)\right]=\left\langle\left\langle C_{0}\left(\boldsymbol{r}, \boldsymbol{r}^{\prime}\right)\right\rangle_{\alpha \beta}\left(\boldsymbol{x}, A, A^{\prime}\right)\right.
$$


where

$$
C_{0}\left(\boldsymbol{r}, \boldsymbol{r}^{\prime}\right)=\int_{V} G_{0}^{*}(\boldsymbol{r}, \boldsymbol{s}) G_{0}\left(\boldsymbol{r}^{\prime}, \boldsymbol{s}\right) M_{0}(\boldsymbol{s}) \mathrm{d} \boldsymbol{s}
$$

is the expectation value of the cross-covariance function and, for any function $F\left(\boldsymbol{r}, \boldsymbol{r}^{\prime}\right)$, the double brackets mean

$$
\begin{aligned}
\left\langle\left\langle F\left(\boldsymbol{r}, \boldsymbol{r}^{\prime}\right)\right\rangle\right\rangle_{\alpha \beta}\left(\boldsymbol{x}, A, A^{\prime}\right) & = \\
\int_{A} \mathrm{~d} \boldsymbol{r} \int_{A^{\prime}} \mathrm{d} \boldsymbol{r}^{\prime}[ & \partial_{n} H_{\alpha}^{*}(\boldsymbol{r}, \boldsymbol{x}) F\left(\boldsymbol{r}, \boldsymbol{r}^{\prime}\right) \partial_{n^{\prime}} H_{\beta}\left(\boldsymbol{r}^{\prime}, \boldsymbol{x}\right) \\
& -H_{\alpha}^{*}(\boldsymbol{r}, \boldsymbol{x}) \partial_{n} F\left(\boldsymbol{r}, \boldsymbol{r}^{\prime}\right) \partial_{n^{\prime}} H_{\beta}\left(\boldsymbol{r}^{\prime}, \boldsymbol{x}\right) \\
& -\partial_{n} H_{\alpha}^{*}(\boldsymbol{r}, \boldsymbol{x}) \partial_{n} F\left(\boldsymbol{r}, \boldsymbol{r}^{\prime}\right) H_{\beta}\left(\boldsymbol{r}^{\prime}, \boldsymbol{x}\right) \\
& \left.+H_{\alpha}^{*}(\boldsymbol{r}, \boldsymbol{x}) \partial_{n} \partial_{n^{\prime}} F\left(\boldsymbol{r}, \boldsymbol{r}^{\prime}\right) H_{\beta}\left(\boldsymbol{r}^{\prime}, \boldsymbol{x}\right)\right] .
\end{aligned}
$$

The perturbation to the image intensity is given by

$$
\begin{aligned}
& \mathbb{E}\left[\delta I_{\alpha \beta}\left(\boldsymbol{x}, A, A^{\prime}\right)\right]=\int_{V} \epsilon(\boldsymbol{s}) \mathcal{K}_{\alpha \beta}^{\epsilon}\left(\boldsymbol{x}, \boldsymbol{s}, A, A^{\prime}\right) \mathrm{d} \boldsymbol{s} \\
& +\int_{V}\left(\delta k^{2 *}\left(\boldsymbol{r}_{\mathrm{s}}\right) \mathcal{K}_{\alpha \beta}^{k}\left(\boldsymbol{x}, \boldsymbol{r}_{\mathrm{s}}, A, A^{\prime}\right)+\delta k^{2}\left(\boldsymbol{r}_{\mathrm{s}}\right) \mathcal{K}_{\beta \alpha}^{k *}\left(\boldsymbol{x}, \boldsymbol{r}_{\mathrm{s}}, A^{\prime}, A\right)\right) \mathrm{d} \boldsymbol{r}_{\mathrm{s}} \\
& +2 \mathrm{i} \omega \int_{V} \boldsymbol{u}\left(\boldsymbol{r}_{\mathrm{s}}\right) \cdot\left(\mathcal{K}_{\alpha \beta}^{u}\left(\boldsymbol{x}, \boldsymbol{r}_{\mathrm{s}}, A, A^{\prime}\right)-\mathcal{K}_{\beta \alpha}^{u *}\left(\boldsymbol{x}, \boldsymbol{r}_{\mathrm{s}}, A^{\prime}, A\right)\right) \mathrm{d} \boldsymbol{r}_{\mathrm{s}},
\end{aligned}
$$

where

$$
\begin{aligned}
& \mathcal{K}_{\alpha \beta}^{\epsilon}\left(\boldsymbol{x}, \boldsymbol{s}, A, A^{\prime}\right)=M_{0}(\boldsymbol{s})\left\langle\left\langle G_{0}^{*}(\boldsymbol{r}, \boldsymbol{s}) G_{0}\left(\boldsymbol{r}^{\prime}, \boldsymbol{s}\right)\right\rangle\right\rangle_{\alpha \beta}\left(\boldsymbol{x}, A, A^{\prime}\right), \\
& \mathcal{K}_{\alpha \beta}^{k}\left(\boldsymbol{x}, \boldsymbol{r}_{\mathrm{s}}, A, A^{\prime}\right)=\left\langle\left\langle C_{0}\left(\boldsymbol{r}_{\mathrm{s}}, \boldsymbol{r}^{\prime}\right) G_{0}^{*}\left(\boldsymbol{r}, \boldsymbol{r}_{\mathrm{s}}\right)\right\rangle\right\rangle_{\alpha \beta}\left(\boldsymbol{x}, A, A^{\prime}\right), \\
& \mathcal{K}_{\alpha \beta}^{u}\left(\boldsymbol{x}, \boldsymbol{r}_{\mathrm{s}}, A, A^{\prime}\right)= \\
& \quad\left\langle\left\langle\nabla\left(\frac{C_{0}\left(\boldsymbol{r}_{\mathrm{s}}, \boldsymbol{r}^{\prime}\right)}{\rho_{0}\left(\boldsymbol{r}_{\mathrm{s}}\right)^{1 / 2} c_{0}\left(\boldsymbol{r}_{\mathrm{s}}\right)}\right) \frac{\rho_{0}\left(\boldsymbol{r}_{\mathrm{s}}\right)^{1 / 2} G_{0}^{*}\left(\boldsymbol{r}, \boldsymbol{r}_{\mathrm{s}}\right)}{c_{0}\left(\boldsymbol{r}_{\mathrm{s}}\right)} \|_{\alpha \beta}\left(\boldsymbol{x}, A, A^{\prime}\right) .\right.\right.
\end{aligned}
$$

The kernels for $\delta k^{2}$ and $\delta k^{2 *}$ can be combined using Eq. (3.23) to obtain kernels for sound-speed $\mathcal{K}_{\alpha \beta}^{c}$, density $\mathcal{K}_{\alpha \beta}^{\rho}$ and attenuation $\mathcal{K}_{\alpha \beta}^{\gamma}$. For example,

$$
\mathbb{E}\left[\delta I_{\alpha \beta}\left(\boldsymbol{x}, A, A^{\prime}\right)\right]=\int_{V} \delta c\left(\boldsymbol{r}_{\mathrm{s}}\right) \mathcal{K}_{\alpha \beta}^{c}\left(\boldsymbol{x}, \boldsymbol{r}_{\mathrm{s}}, A, A^{\prime}\right) \mathrm{d} \boldsymbol{r}_{\mathrm{s}}
$$

with

$$
\begin{aligned}
\mathcal{K}_{\alpha \beta}^{c}\left(\boldsymbol{x}, \boldsymbol{r}_{\mathrm{s}}, A, A^{\prime}\right)= & -\frac{2\left(\omega^{2}-2 \mathrm{i} \omega \gamma\right)}{c_{0}^{3}\left(\boldsymbol{r}_{\mathrm{s}}\right)} \mathcal{K}_{\alpha \beta}^{k}\left(\boldsymbol{x}, \boldsymbol{r}_{\mathrm{s}}, A, A^{\prime}\right) \\
& -\frac{2\left(\omega^{2}+2 \mathrm{i} \omega \gamma\right)}{c_{0}^{3}\left(\boldsymbol{r}_{\mathrm{s}}\right)} \mathcal{K}_{\beta \alpha}^{k *}\left(\boldsymbol{x}, \boldsymbol{r}_{\mathrm{s}}, A^{\prime}, A\right)
\end{aligned}
$$




\subsubsection{Choice of the source covariance}

In order to be able to compute the above kernels, one still need to choose the source covariance function $M_{0}$ in order to define the reference cross-covariance $C_{0}$ using Eq. (3.34). One possibility is to place the sources at a single depth, a few hundred kilometers below the solar surface.

Another possibility is to choose a source covariance of the form

$$
M_{0}(\boldsymbol{r}, \omega)=\Pi(\omega) \frac{\gamma(\boldsymbol{r}, \omega)}{c_{0}^{2}(\boldsymbol{r})},
$$

where $\Pi(\omega)$ is the source power spectrum (see Gizon et al. 2017). This choice implies

$$
C_{0}\left(\boldsymbol{r}, \boldsymbol{r}^{\prime}, \omega\right)=\frac{\Pi(\omega)}{2 \omega} \operatorname{Im} G_{0}\left(\boldsymbol{r}^{\prime}, \boldsymbol{r}, \omega\right)+\text { surface term }
$$

The surface term depends on the boundary condition. It vanishes for a Dirichlet boundary condition (free surface), while it remains for radiative boundary conditions (e.g. Sommerfeld). Below the acoustic cutoff frequency, modes are trapped well below the observational and computational boundaries and the surface term is negligible. In this paper we use Eq. (3.42) in the convection zone and switch off the sources above the photosphere. By doing so, the surface term in Eq. (3.43) vanishes.

\subsection{Noise}

To compute the noise level, we compute the variance of the image intensity in the absence of scatterers:

$$
\begin{gathered}
\operatorname{Var}\left[I_{\alpha \beta, 0}(\boldsymbol{x})\right]=\operatorname{Var}\left[\int K_{\alpha, 0}^{*}(\boldsymbol{x}, \boldsymbol{s}) K_{\beta, 0}\left(\boldsymbol{x}, \boldsymbol{s}^{\prime}\right) S^{*}(\boldsymbol{s}) S\left(\boldsymbol{s}^{\prime}\right) \mathrm{d} \boldsymbol{s} \mathrm{d} \boldsymbol{s}^{\prime}\right] \\
=\int_{V^{4}} K_{\alpha, 0}^{*}\left(\boldsymbol{x}, \boldsymbol{s}_{1}\right) K_{\beta, 0}\left(\boldsymbol{x}, \boldsymbol{s}_{1}^{\prime}\right) K_{\alpha, 0}\left(\boldsymbol{x}, \boldsymbol{s}_{2}\right) K_{\beta, 0}^{*}\left(\boldsymbol{x}, \boldsymbol{s}_{2}^{\prime}\right) \\
\times M_{4}\left(\boldsymbol{s}_{1}, \boldsymbol{s}_{1}^{\prime}, \boldsymbol{s}_{2}, \boldsymbol{s}_{2}^{\prime}\right) \mathrm{d} \boldsymbol{s}_{1} \mathrm{~d} \boldsymbol{s}_{1}^{\prime} \mathrm{d} \boldsymbol{s}_{2} \mathrm{~d} \boldsymbol{s}_{2}^{\prime} \\
-\left|\int_{V^{2}} K_{\alpha, 0}^{*}(\boldsymbol{x}, \boldsymbol{s}) K_{\beta, 0}\left(\boldsymbol{x}, \boldsymbol{s}^{\prime}\right) \mathbb{E}\left[S^{*}(\boldsymbol{s}) S\left(\boldsymbol{s}^{\prime}\right)\right] \mathrm{d} \boldsymbol{s} \mathrm{d} \boldsymbol{s}^{\prime}\right|^{2},
\end{gathered}
$$

where

$$
M_{4}\left(s_{1}, s_{1}^{\prime}, s_{2}, s_{2}^{\prime}\right)=\mathbb{E}\left[S^{*}\left(s_{1}\right) S\left(s_{1}^{\prime}\right) S\left(s_{2}\right) S^{*}\left(s_{2}^{\prime}\right)\right] .
$$

Under the (very reasonable) assumption that $S$ is a realization drawn from a Gaussian random process, the fourth-order moment is the sum of products of the second-order moments:

$$
\begin{aligned}
M_{4}\left(\boldsymbol{s}_{1}, \boldsymbol{s}_{1}^{\prime}, \boldsymbol{s}_{2}, \boldsymbol{s}_{2}^{\prime}\right) & =\mathbb{E}\left[S^{*}\left(\boldsymbol{s}_{1}\right) S\left(\boldsymbol{s}_{1}^{\prime}\right)\right] \mathbb{E}\left[S\left(\boldsymbol{s}_{2}\right) S^{*}\left(\boldsymbol{s}_{2}^{\prime}\right)\right] \\
& +\mathbb{E}\left[S^{*}\left(\boldsymbol{s}_{1}\right) S\left(\boldsymbol{s}_{2}\right)\right] \mathbb{E}\left[S\left(\boldsymbol{s}_{1}^{\prime}\right) S^{*}\left(\boldsymbol{s}_{2}^{\prime}\right)\right] \\
& +\mathbb{E}\left[S^{*}\left(\boldsymbol{s}_{1}\right) S^{*}\left(\boldsymbol{s}_{2}^{\prime}\right)\right] \mathbb{E}\left[S\left(\boldsymbol{s}_{2}\right) S\left(\boldsymbol{s}_{1}^{\prime}\right)\right]
\end{aligned}
$$


The first term in $M_{4}$ cancels out the squared term in Eq. 3.44). The third term is zero as the frequencies are uncorrelated. Thus,

$$
\begin{aligned}
\operatorname{Var}\left[I_{\alpha \beta, 0}(\boldsymbol{x})\right]= & \int_{V^{4}} K_{\alpha, 0}^{*}\left(\boldsymbol{x}, \boldsymbol{s}_{1}\right) K_{\beta, 0}\left(\boldsymbol{x}, \boldsymbol{s}_{1}^{\prime}\right) K_{\alpha, 0}\left(\boldsymbol{x}, \boldsymbol{s}_{2}\right) K_{\beta, 0}^{*}\left(\boldsymbol{x}, \boldsymbol{s}_{2}^{\prime}\right) \\
& \times \mathbb{E}\left[S^{*}\left(\boldsymbol{s}_{1}\right) S\left(\boldsymbol{s}_{2}\right)\right] \mathbb{E}\left[S\left(\boldsymbol{s}_{1}^{\prime}\right) S^{*}\left(\boldsymbol{s}_{2}^{\prime}\right)\right] \mathrm{d} \boldsymbol{s}_{1} \mathrm{~d} \boldsymbol{s}_{1}^{\prime} \mathrm{d} \boldsymbol{s}_{2} \mathrm{~d} \boldsymbol{s}_{2}^{\prime} \\
= & \int_{V}\left|K_{\alpha, 0}(\boldsymbol{x}, \boldsymbol{s})\right|^{2} M(\boldsymbol{s}) \mathrm{d} \boldsymbol{s} \int_{V}\left|K_{\beta, 0}(\boldsymbol{x}, \boldsymbol{s})\right|^{2} M(\boldsymbol{s}) \mathrm{d} \boldsymbol{s} \\
= & \mathbb{E}\left[I_{\alpha, 0}(\boldsymbol{x})\right] \mathbb{E}\left[I_{\beta, 0}(\boldsymbol{x})\right] .
\end{aligned}
$$

When $\alpha=\beta$, the standard deviation of $I_{\alpha, 0}$ is equal to its expectation value. This is because the probability density function of $I_{\alpha, 0}$ is a $\chi^{2}$ with two degrees of freedom, i.e. an exponential distribution.

\subsection{Average over frequencies}

In order to increase the signal-to-noise ratio, one usually averages the image intensity over a range of frequencies $\left[\omega_{0}-\Delta \omega / 2, \omega_{0}+\Delta \omega / 2\right]$. For an observation duration $T$, this interval will contain $N=\Delta \omega T / 2 \pi$ independent frequencies. by

The frequency-averaged perturbation to the image intensity (i.e. the signal) is denoted

$$
\left\langle\delta I_{\alpha \beta}(\boldsymbol{x})\right\rangle=\frac{1}{N} \sum_{i=1}^{N} \delta I_{\alpha \beta}\left(\boldsymbol{x}, \omega_{i}\right) .
$$

The variance of the noise in the average image intensity is then given by

$$
\begin{aligned}
\operatorname{Var}\left\langle I_{\alpha \beta, 0}(\boldsymbol{x})\right\rangle & =\operatorname{Var}\left(\frac{1}{N} \sum_{i=1}^{N} I_{\alpha \beta, 0}\left(\boldsymbol{x}, \omega_{i}\right)\right) \\
& =\frac{1}{N^{2}} \sum_{i=1}^{N} \operatorname{Var} I_{\alpha \beta, 0}\left(\boldsymbol{x}, \omega_{i}\right) \\
& =\frac{1}{N}\left\langle\operatorname{Var} I_{\alpha \beta, 0}(\boldsymbol{x})\right\rangle,
\end{aligned}
$$

since the noise in the data at different frequencies is uncorrelated.

The expected signal-to-noise ratio is thus

$$
\operatorname{SNR}(\boldsymbol{x})=\frac{\left|\mathbb{E}\left\langle\delta I_{\alpha \beta}(\boldsymbol{x})\right\rangle\right|}{\sqrt{\operatorname{Var}\left\langle I_{\alpha \beta, 0}(\boldsymbol{x})\right\rangle}}=\frac{\sqrt{N}\left|\mathbb{E}\left\langle\delta I_{\alpha \beta}(\boldsymbol{x})\right\rangle\right|}{\sqrt{\left\langle\mathbb{E}\left[I_{\alpha, 0}(\boldsymbol{x})\right] \mathbb{E}\left[I_{\beta, 0}(\boldsymbol{x})\right]\right\rangle}} .
$$

The number of available frequencies $N$ within a fixed frequency band $\Delta \omega$ is proportional to the observation duration $T$, hence the noise level goes like $T^{-1 / 2}$. Provided that the frequency interval $\Delta \omega$ is small with respect to the variations of the signal, then the signalto-noise ratio will increase like $T^{1 / 2}$. 


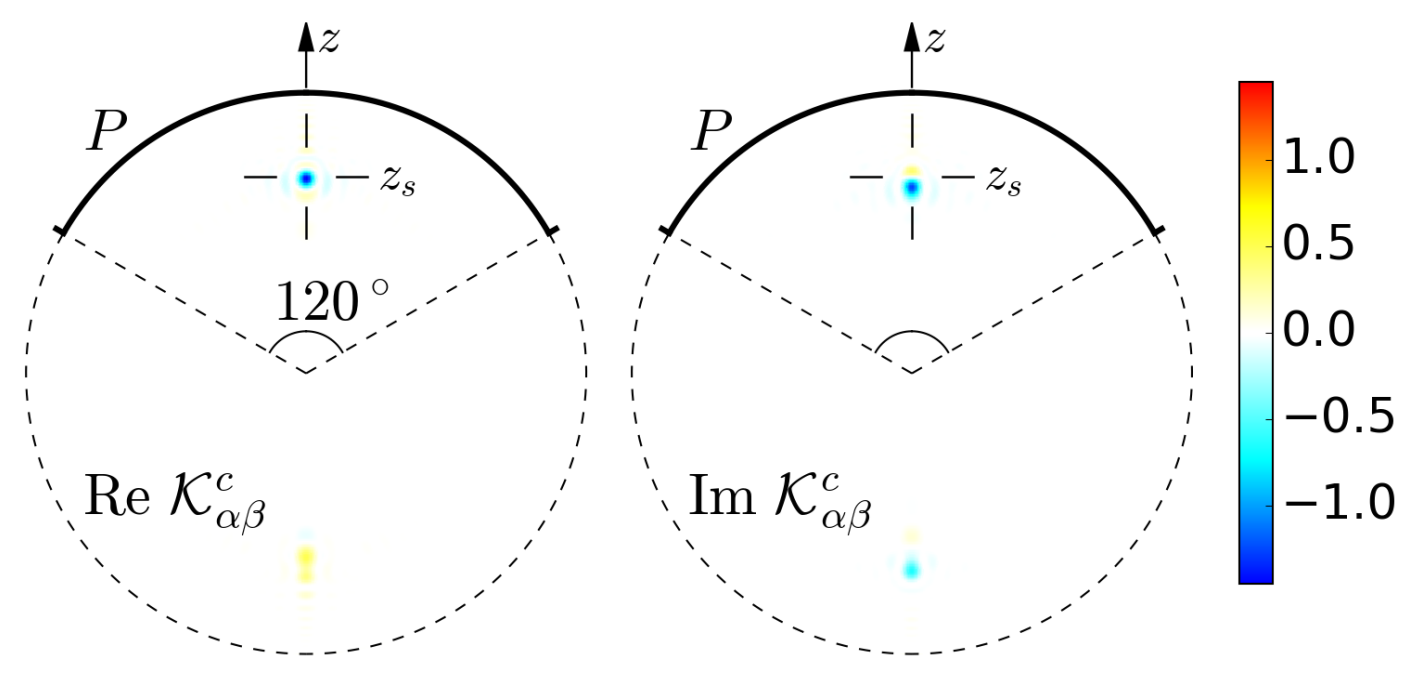

Figure 3.2: Meridional slice through the sound-speed kernel $\mathcal{K}_{\alpha \beta}^{c}\left(\boldsymbol{x}, \boldsymbol{r}_{\mathrm{s}}\right)$ computed in Model $\mathrm{S}$ at a single frequency of $3 \mathrm{mHz}$, in units of $10^{-34} \mathrm{~kg} \mathrm{~m}^{-3} \mathrm{~s}^{-3}$. Both the real (left panel) and imaginary (right panel) parts of the kernel are shown. The scatterer at $z_{\mathrm{s}}=0.7 \mathrm{R}_{\odot}$ is indicated by the crosses. The observation pupil $P$ is a polar cap of full angular size $120^{\circ}$. Notice the ghost values at the antipode result from the reflection of waves at the surface due to the rapid drop of the density.

\subsection{Example computations}

In order to illustrate the theory, we compute holographic images in the presence of soundspeed perturbations at different depths and calculate the corresponding signal-to-noise ratios.

\subsubsection{Reference Green's function}

The main input quantity required to compute PB integrals is the reference Green's function (Eq. 3.10). Here it is computed in the frequency domain using the sphericallysymmetric standard solar Model S (Christensen-Dalsgaard et al. 1996). The wave attenuation model is taken from Gizon et al. (2017). Below $5.3 \mathrm{mHz}$, we have $\gamma=\gamma_{0}\left|\omega / \omega_{0}\right|^{5.77}$, where $\gamma_{0} / 2 \pi=4.29 \mu \mathrm{Hz}$ and $\omega_{0} / 2 \pi=3 \mathrm{mHz}$. Above $5.3 \mathrm{mHz}, \gamma / 2 \pi=125 \mu \mathrm{Hz}$ is kept constant. The radiation boundary condition defined by Eq. (3.7) is applied at the computational boundary located $500 \mathrm{~km}$ above the solar surface with the local wavenumber $k_{n}$ (where $H=105 \mathrm{~km}$ ). The wave equation is solved using the finite-element solver Montjoie (Duruflé 2006, Gizon et al. 2017).

The reference Green's function only depends on the angular distance $\Theta$ between the two points at radii $r$ and $r^{\prime}$. To speed up the computations, we place one of the points on the polar axis and compute the axisymmetric component of the Green's function 


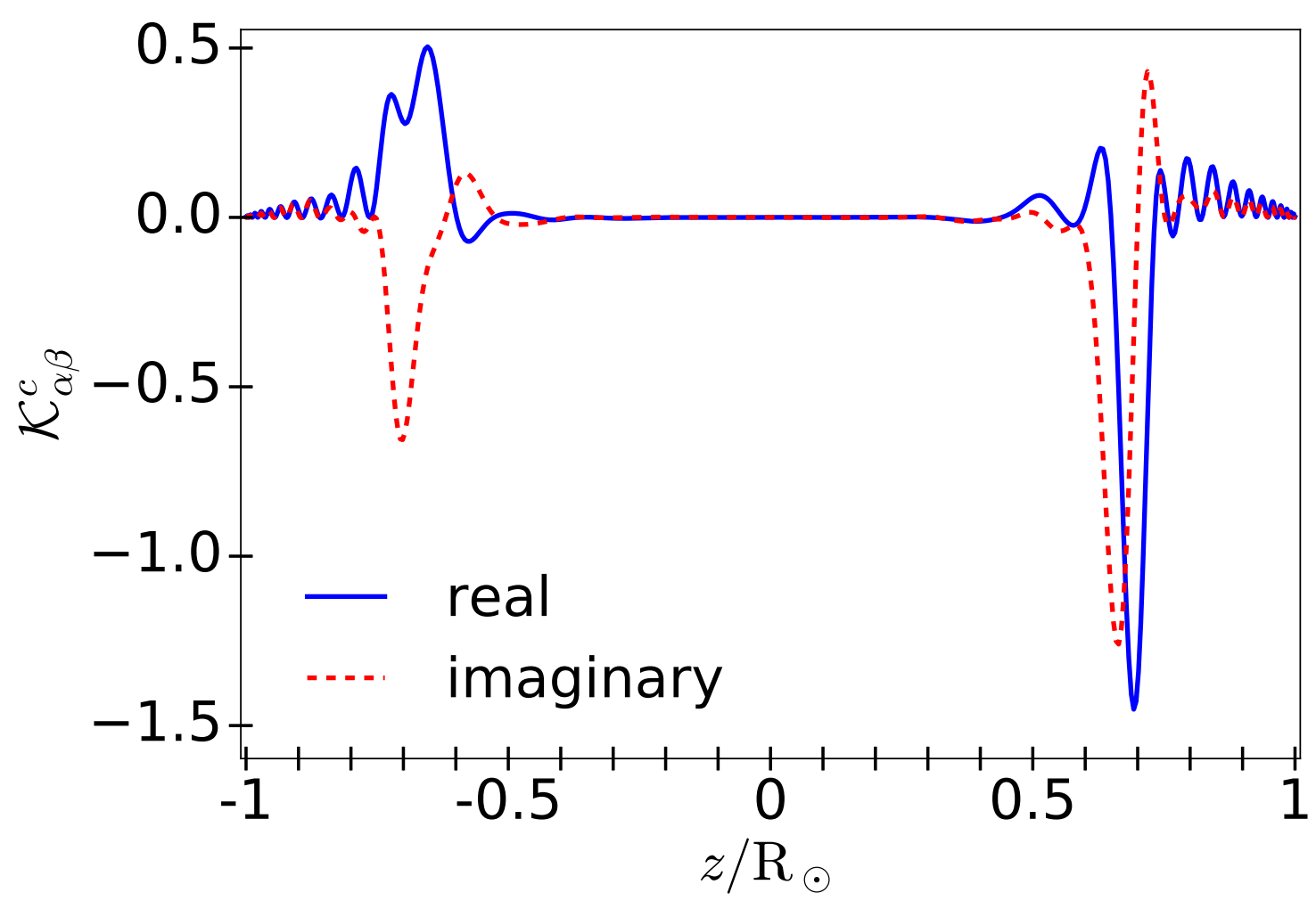

Figure 3.3: Cut along the $z$ axis through the sound-speed kernel from Fig. 3.2. The scatterer is at $z_{\mathrm{s}}=0.7 \mathrm{R}_{\odot}$.

$G_{l}^{m=0}\left(r, r^{\prime}, \omega\right)$ at each spherical harmonic degree $l$, to obtain:

$$
G_{0}\left(\boldsymbol{r}, \boldsymbol{r}^{\prime}, \omega\right) \simeq \sum_{l=0}^{l_{\max }} G_{l}^{m=0}\left(r, r^{\prime}, \omega\right) P_{l}(\cos \Theta),
$$

where we use an approximate equality because the sum is truncated at $l_{\max }=300$.

\subsubsection{Sound-speed kernels}

The sound-speed kernel is computed using Eq. 3.41 and the definition of $\mathcal{K}_{\alpha \beta}^{k}$. One needs to evaluate two surface integrals, which can be computed analytically via a decomposition of all quantities into spherical harmonic coefficients (Fournier et al.|2018).

Figure 3.2 shows a sound-speed kernel $\mathcal{K}_{\alpha \beta}^{c}$ at a single frequency of $3 \mathrm{mHz}$. The pupil $P$ is a polar cap of angular size $120^{\circ}$ and the wave propagators $H_{\alpha}$ and $H_{\beta}$ are given in Table 3.2. As expected the kernel peaks around the scatterer position at $z=0.7 \mathrm{R}_{\odot}$. A cut along the polar axis is shown in Fig. 3.3, the kernel width is about half the local wavelength. In addition, we find ghost values at the antipode. 

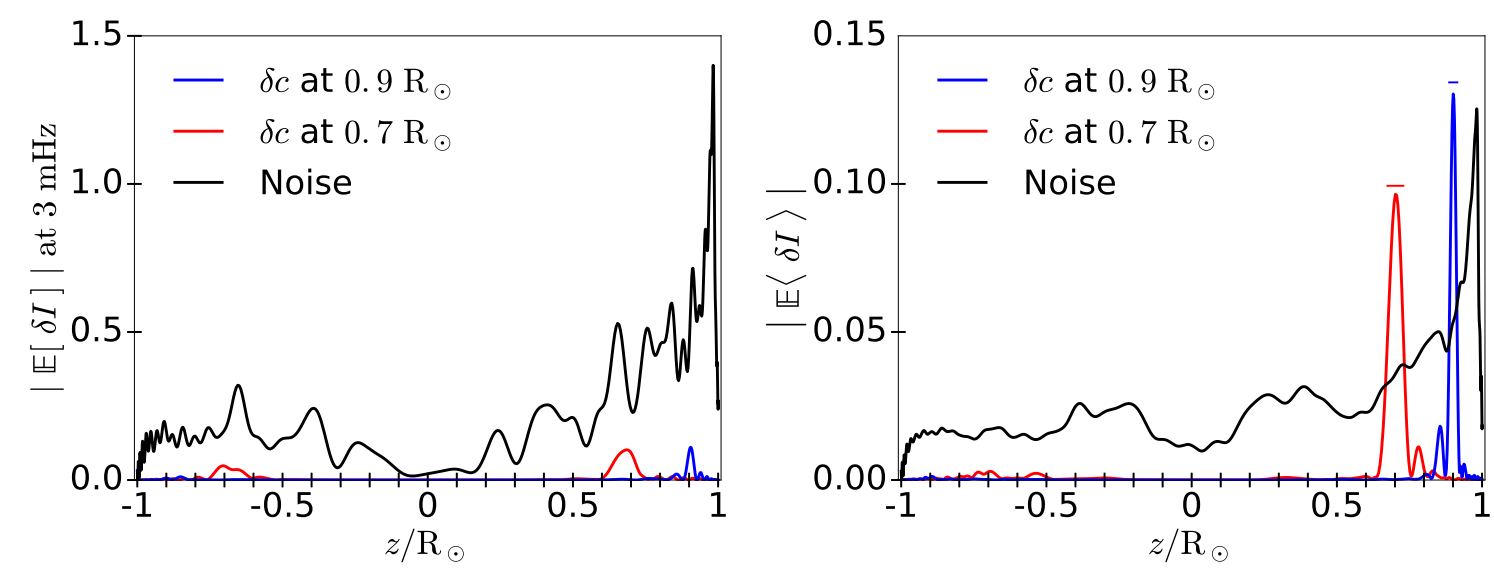

Figure 3.4: Left panel: Image intensity $\left|\mathbb{E}\left[\delta I_{\alpha \beta}(\boldsymbol{x})\right]\right|$ at a single frequency of $3 \mathrm{mHz}$, displayed along the $z$-axis (at $\boldsymbol{x}=z \hat{\mathbf{z}}$ ). The sound-speed perturbation (see Eq. 3.54) is placed at two different positions along the $z$-axis, $z_{\mathrm{s}}=0.7 \mathrm{R}_{\odot}($ red $)$ and $0.9 \mathrm{R}_{\odot}$ (blue). The standard deviation of the noise $\sqrt{\operatorname{Var}\left\langle I_{\alpha \beta, 0}(\boldsymbol{x})\right\rangle}$ is given by the black curve. Note that the jagged aspect of the curves is not due to numerical inaccuracies. Right panel: Image intensity and noise level after averaging over 101 frequencies uniformly distributed in the interval from 2.75 to $3.25 \mathrm{mHz}$. The frequency resolution is $5 \mu \mathrm{Hz}$, implying an observation duration of $T=55.5 \mathrm{~h}$. A horizontal line segment is plotted at each depth to mark half of the local wavelength.

\subsubsection{Signal}

At position $\boldsymbol{r}_{\mathrm{s}}=z_{\mathrm{s}} \hat{\mathbf{z}}$ along the polar axis, we consider a localized increase in sound speed of $10 \%$ over a volume $V_{\mathrm{s}}$, such that the signal (Eq. 3.40 may be written as

$$
\mathbb{E}\left[\delta I_{\alpha \beta}(\boldsymbol{x})\right] \simeq 0.1 V_{\mathrm{s}} c_{0}\left(\boldsymbol{r}_{\mathrm{s}}\right) \mathcal{K}_{\alpha \beta}^{c}\left(\boldsymbol{x}, \boldsymbol{r}_{\mathrm{s}}\right) .
$$

The volume $V_{\mathrm{s}}$ is that of a ball of diameter $\lambda\left(r_{\mathrm{s}}\right) / 2=\pi /\left[\operatorname{Re} k\left(r_{\mathrm{s}}, \omega_{0}\right)\right]$ with $\omega_{0} / 2 \pi=$ $3 \mathrm{mHz}$. This is an approximate but much faster way to compute the effect of a perturbation of volume comparable to the highest possible holographic resolution. It has been checked that the answer does not differ significantly from the one obtained by integrating numerically the kernel over the ball of volume $V_{\mathrm{s}}$. For reference, note that $\lambda / 2=38 \mathrm{Mm}$ at $r=0.7 \mathrm{R}_{\odot}$ and $\lambda / 2=20 \mathrm{Mm}$ at $r=0.9 \mathrm{R}_{\odot}$.

Figure 3.4 shows the signal $\left|\mathbb{E}\left[\delta I_{\alpha \beta}(\boldsymbol{x})\right]\right|$ for sound-speed perturbations located at two different depths $z_{\mathrm{s}}=0.7 \mathrm{R}_{\odot}$ (red curve) and $0.9 \mathrm{R}_{\odot}$ (blue curve). The pupil $P$ and the wave propagators are the same as those of Fig. 3.2. The left panel of Fig. 3.4 shows the results at a single frequency of $3 \mathrm{mHz}$. With only one frequency, the signal peaks close to the scattering location but the spatial resolution is relatively poor, with a ghost on the far side. To demonstrate the benefits of averaging, the right panel shows the signal after averaging over 101 frequencies uniformly distributed in the interval $2.75-3.25 \mathrm{mHz}$. The frequency resolution $5 \mu \mathrm{Hz}$ corresponds to an observation duration $T=55.5 \mathrm{~h}$. Averaging over frequencies improves the spatial resolution which approaches $\lambda / 2$ and the ghost is suppressed. A horizontal line segment is plotted on the right panel at each depth to mark half the local wavelength. 


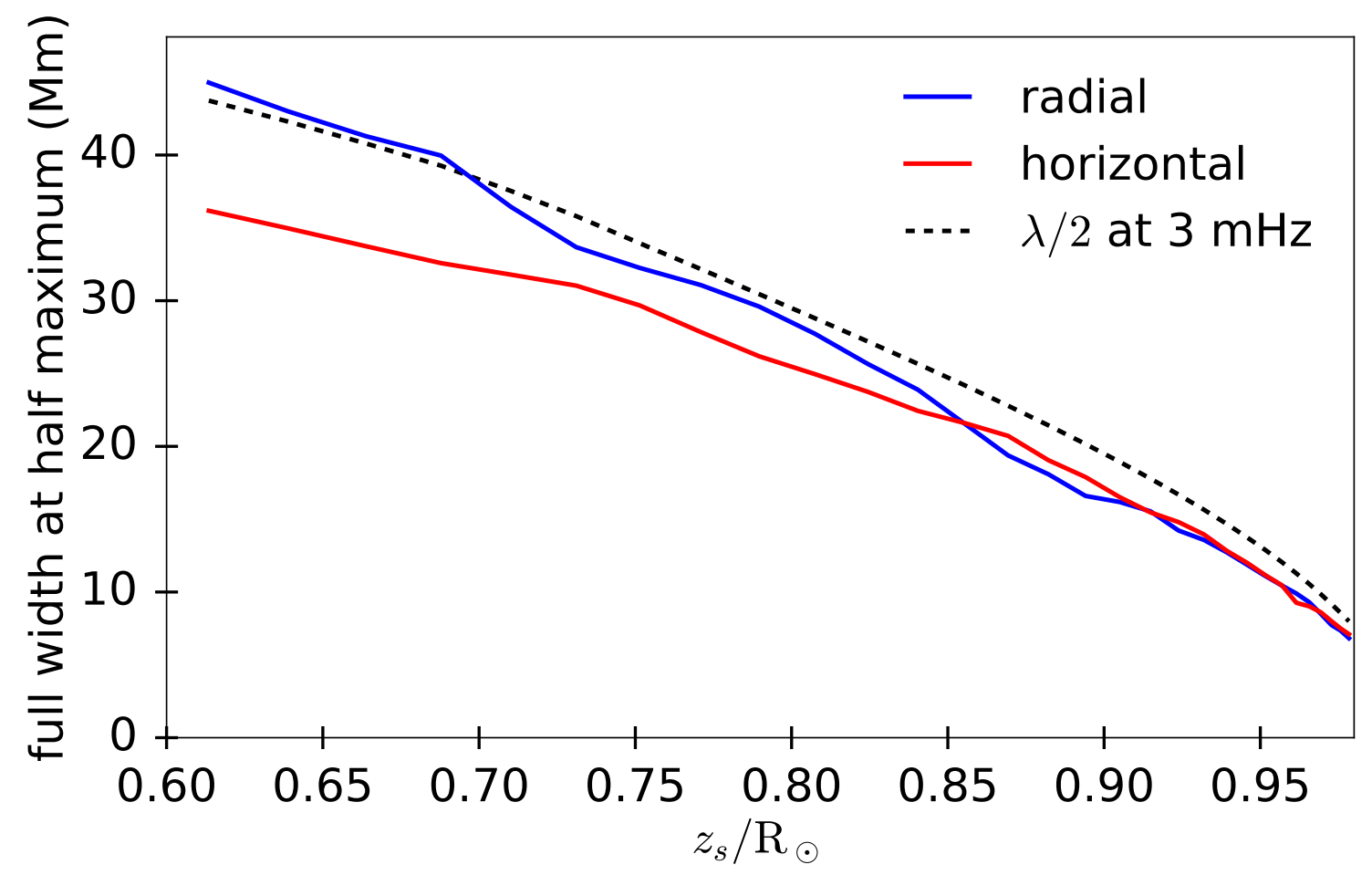

Figure 3.5: Radial and horizontal widths of the frequency-averaged sound-speed kernel $\left|\left\langle\mathcal{K}^{c}\right\rangle\right|$ as functions of scattering position. These are close to half the local wavelength at $3 \mathrm{mHz}$ (dotted line).

As seen in Fig. 3.5 the spatial extent of the frequency-averaged kernels is approximately $\lambda / 2$ in both the radial and horizontal directions, for all scattering points in the range $0.6<z_{s} / \mathrm{R}_{\odot}<0.98$. Thus helioseismic holography is a diffraction-limited imaging technique as suggested by Lindsey and Braun (1997).

\subsubsection{Noise}

The noise is obtained from Eq. (3.47), which requires the computation of $\mathbb{E}\left[I_{\alpha, 0}\right]$ and $\mathbb{E}\left[I_{\beta, 0}\right]$ using Eq. (3.33). The reference cross-covariance $C_{0}$ is precomputed. The double surface integral is evaluated in a similar way as for the kernel computations.

For a frequency of $3 \mathrm{mHz}$ the left panel of Fig. 3.4 (black curve) shows the noise level, together with the signal described in the previous section. The jagged aspect of the noise variations with position is not due to numerical inaccuracies but to the details of the Green's function. As shown in the right panel of Fig. 3.4, the noise level goes down by a factor of about ten after averaging over 101 frequencies, and varies more smoothly with depth.

Braun and Birch (2008a) studied the noise level in observed travel times measured from LB holography. These measurements, however, include contributions from supergranulation and so are not directly comparable to what is shown in Fig. 3.4. 


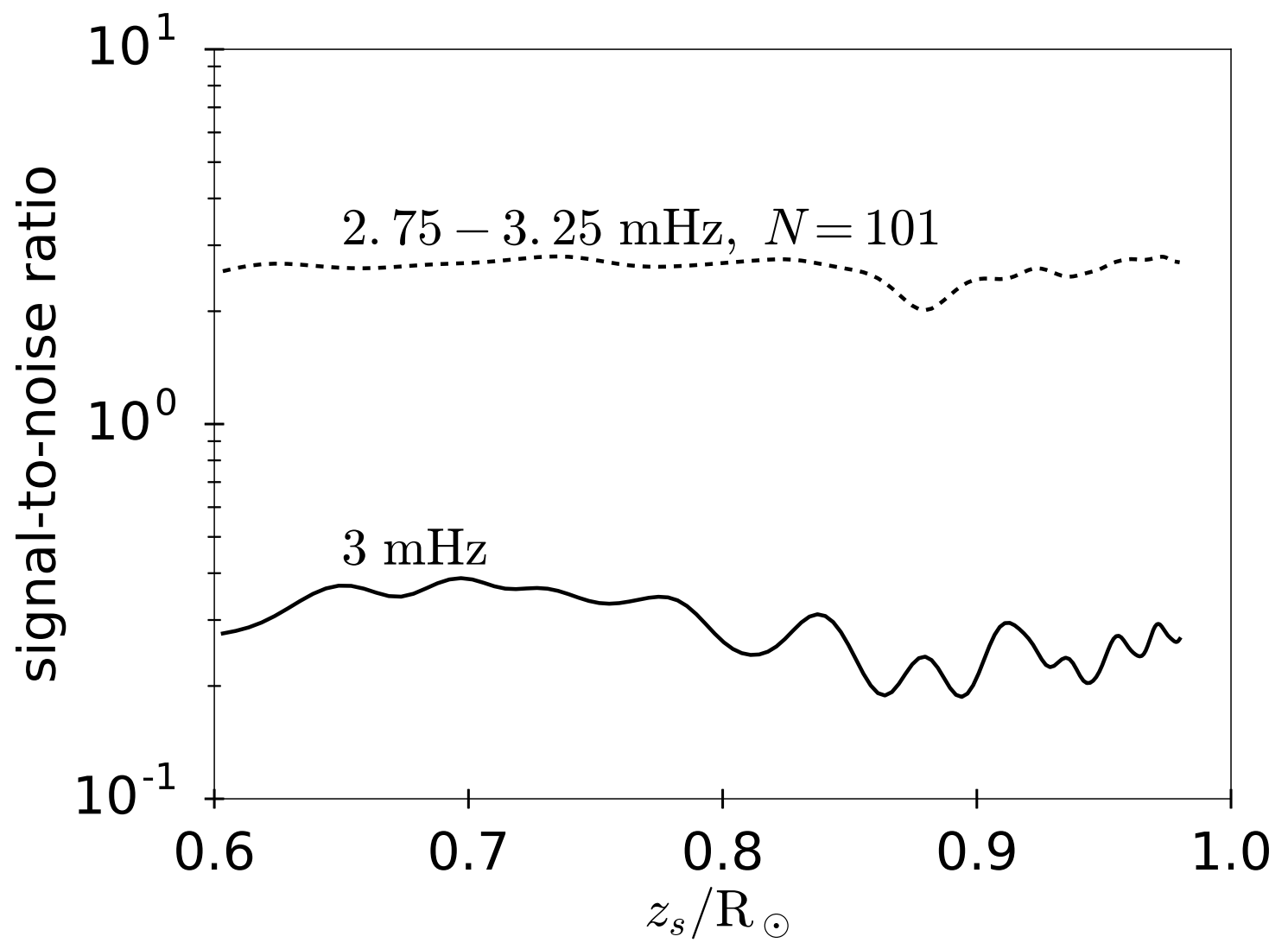

Figure 3.6: Signal-to-noise ratio in PB image intensity for a $10 \%$ sound-speed perturbation over a volume $V_{\mathrm{s}}\left(z_{\mathrm{s}}\right)$ placed at $z_{\mathrm{s}}$ along the polar axis (Eq. 3.54). The results are shown at a single frequency of $3 \mathrm{mHz}$ (solid) and after averaging over 101 frequencies in the interval from 2.75 to $3.25 \mathrm{mHz}$ (dashed).

\subsubsection{Signal-to-noise ratio}

Figure 3.6 shows the signal-to-noise ratio as a function of scatterer location. We recall that the sound-speed perturbation is specified by Eq. (3.54) and is the same as in Sect. 3.8.3. The results are shown at a single frequency of $3 \mathrm{mHz}$ and after averaging over 101 frequencies in the interval $2.75-3.25 \mathrm{mHz}$. After averaging, the signal-to-noise ratio is above 2 and is roughly independent of depth in the range $0.6<z_{\mathrm{s}} / \mathrm{R}_{\odot}<0.98$ for a pupil of angular size $120^{\circ}$. Note that the ghost at $-z_{\mathrm{s}}$ is much below the noise level.

We find that both signal and noise vary rapidly with frequency for deep located soundspeed scatterers. Figure 3.7 shows an example of a sound-speed scatterer located at $z_{\mathrm{s}}=$ $0.7 \mathrm{R}_{\odot}$. Strong frequency variations in signal and noise are evident for frequencies below $3.5 \mathrm{mHz}$. This can be understood as follows. Low-frequency modes have narrowlypeaked power spectra due to their long lifetimes. At these low frequencies, the amplitude of the kernel function and the noise will change rapidly when the frequency coincides with a particular mode frequency. Additionally, the kernel function may not peak exactly at the sound-speed scatterer position when only a few modes contribute to the kernel function. Figure 3.8 shows the signal-to-noise ratio as a function of frequency for a sound-speed scatterer located at $z_{\mathrm{s}}=0.9 \mathrm{R}_{\odot}$. For this target depth closer to the surface, the rapid 
variations disappear above $3 \mathrm{mHz}$, due to the larger contribution of high-degree modes which are not resolved in frequency space because of their short lifetimes.

The kernel function at frequency $2.4000 \mathrm{mHz}$ for $z_{\mathrm{s}}=0.7 \mathrm{R}_{\odot}$ is shown in Fig. 3.9; this particular frequency corresponds to the peak marked in Fig. 3.7 with a red dot. We see that this kernel is much less localized around the scattering point than the kernel at 3 $\mathrm{mHz}$ (Fig. 3.2).

\subsection{Conclusion}

We derived a framework for computing the expected signal and the noise level in PB helioseismic holography. The same framework could be used to interpret LB data, including phase-sensitive data.

PB holography requires knowledge of the wave field, $\psi=\rho^{1 / 2} c^{2} \nabla \cdot \boldsymbol{\xi}$, and its normal derivative, $\partial_{n} \psi$, on the solar surface. With this definition of $\psi$, the Green's function used in the definition of PB integrals solves a well-defined Helmholtz-like equation, which we solve numerically (Gizon et al. 2017). The need for finite-frequency Green's functions was demonstrated in LB holography by Pérez Hernández and González Hernández (2010). In the numerical examples shown in the previous section, we assumed that we have full knowledge of $\partial_{n} \psi$ on the surface. In practice, we do not observe directly the normal derivative of the wave field; it must be approximated. According to complementary simulations (not shown here), this can be achieved by using the approximate outgoing radiation condition $\partial_{n} \psi=\mathrm{i} k_{n} \psi$ derived by Barucq et al. (2018).

We found that, for a sufficiently large pupil, scatterers can be imaged at a resolution that is very close to half the local wavelength, $\lambda / 2$. This confirms the claim by Lindsey and Braun (1997, 2000a) that helioseismic holography is diffraction-limited. In that sense, helioseismic holography is superior to deep-focusing time-distance helioseismology, which gives lower spatial resolution (Munk|2001, Pourabdian et al. 2018).

For large pupils, we found that the signal-to-noise ratio in PB images does not vary much with depth in the convection zone, when a perturbation in sound-speed fills a volume corresponding to the holographic resolution.

Averaging over frequencies improves the signal-to-noise ratio. For a scatterer at the bottom of the convection zone, the signal and the noise vary smoothly with frequency above $4 \mathrm{mHz}$ (see Fig. 3.7). At lower frequencies, however, the signal varies rapidly with frequency (due to contributions from individual long-lived p modes) and it is not obvious how the signal should be averaged. A specific analysis of low-frequency data is required, especially for deep scatterers.

We found that the signal-to-noise ratio in $\mathrm{PB}$ holography is maximum around $3.7 \mathrm{mHz}$ for $z_{\mathrm{s}}=0.7 \mathrm{R}_{\odot}\left(\right.$ resp. at $4.3 \mathrm{mHz}$ for $z_{\mathrm{s}}=0.9 \mathrm{R}_{\odot}$ ). There is no indication in our calculations that there is a benefit in using the frequencies above the acoustic cutoff (unlike predictions by Ruzmaikin and Lindsey 2003, for phase sensitive holography). The signalto-noise ratio drops to very small values above $5 \mathrm{mHz}$. One may ask if this drop is somehow compensated by the increase in spatial resolution at high frequencies. The answer is negative. Our calculations indicate that noise has a horizontal correlation length that is about half the local wavelength. Far too few independent measurements are available at high frequencies to recover a decent signal-to-noise ratio by horizontal spatial averaging. 

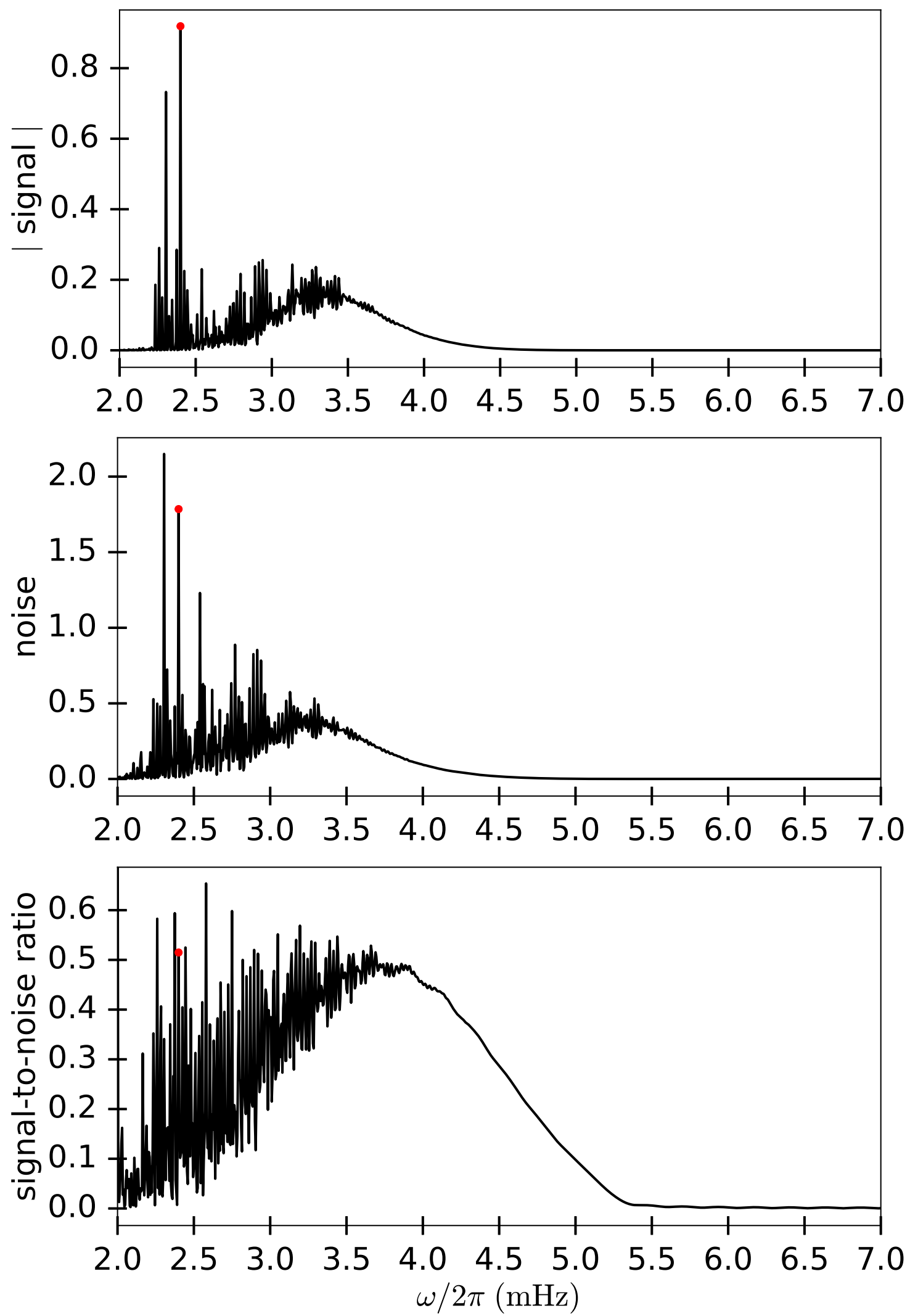

Figure 3.7: Signal, noise, and signal-to-noise ratio as function of frequency for a soundspeed scatterer located at $z_{\mathrm{s}}=0.7 \mathrm{R}_{\odot}$. Here we show the result for a frequency range of 2 to $7 \mathrm{mHz}$. The rapid changes are not due to numerical inaccuracies. The red dots mark the spikes in the signal and the noise at $2.4000 \mathrm{mHz}$. 


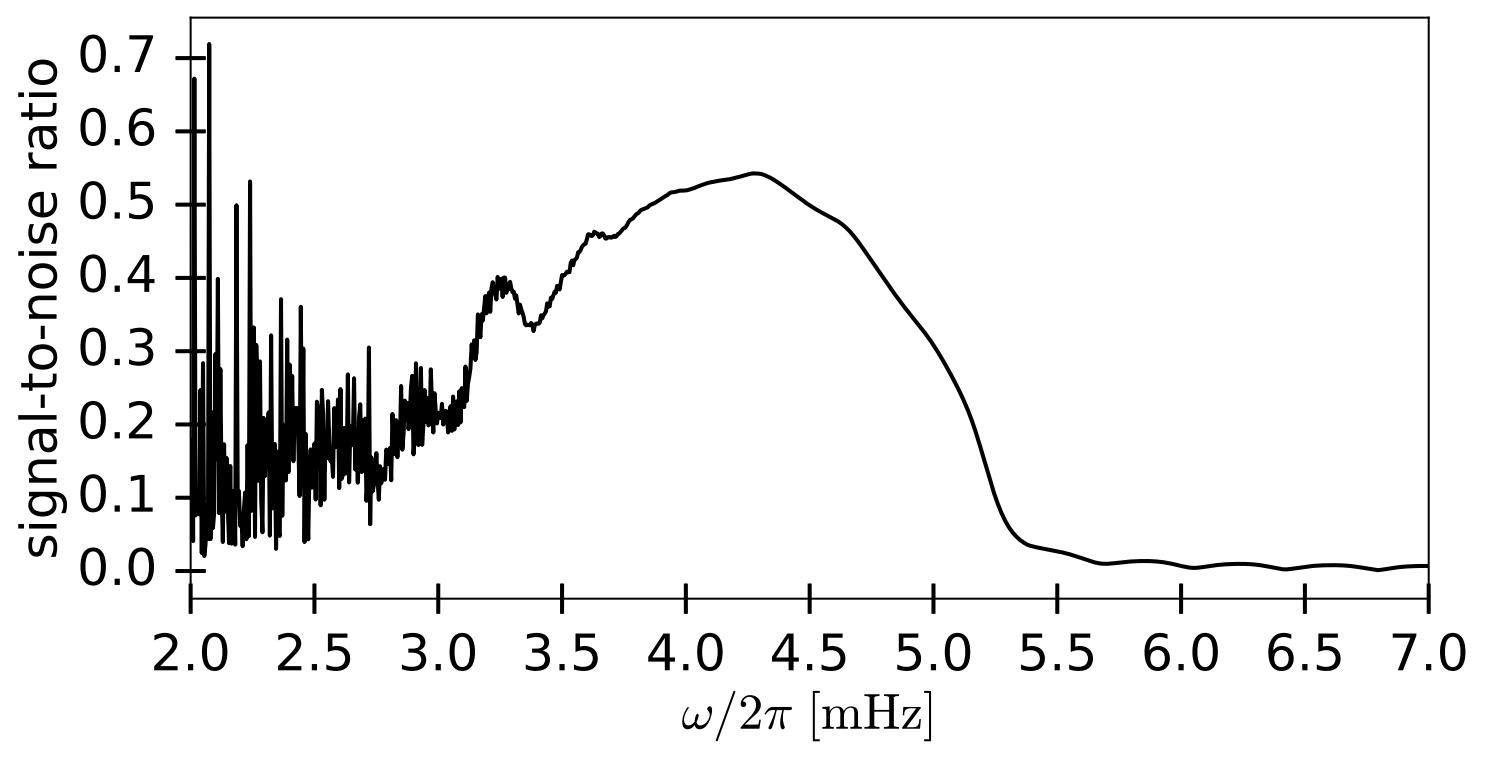

Figure 3.8: Signal-to-noise ratio as in Fig. 3.7, but for a sound-speed scatterer located closer to the surface at $z_{\mathrm{s}}=0.9 \mathrm{R}_{\odot}$.
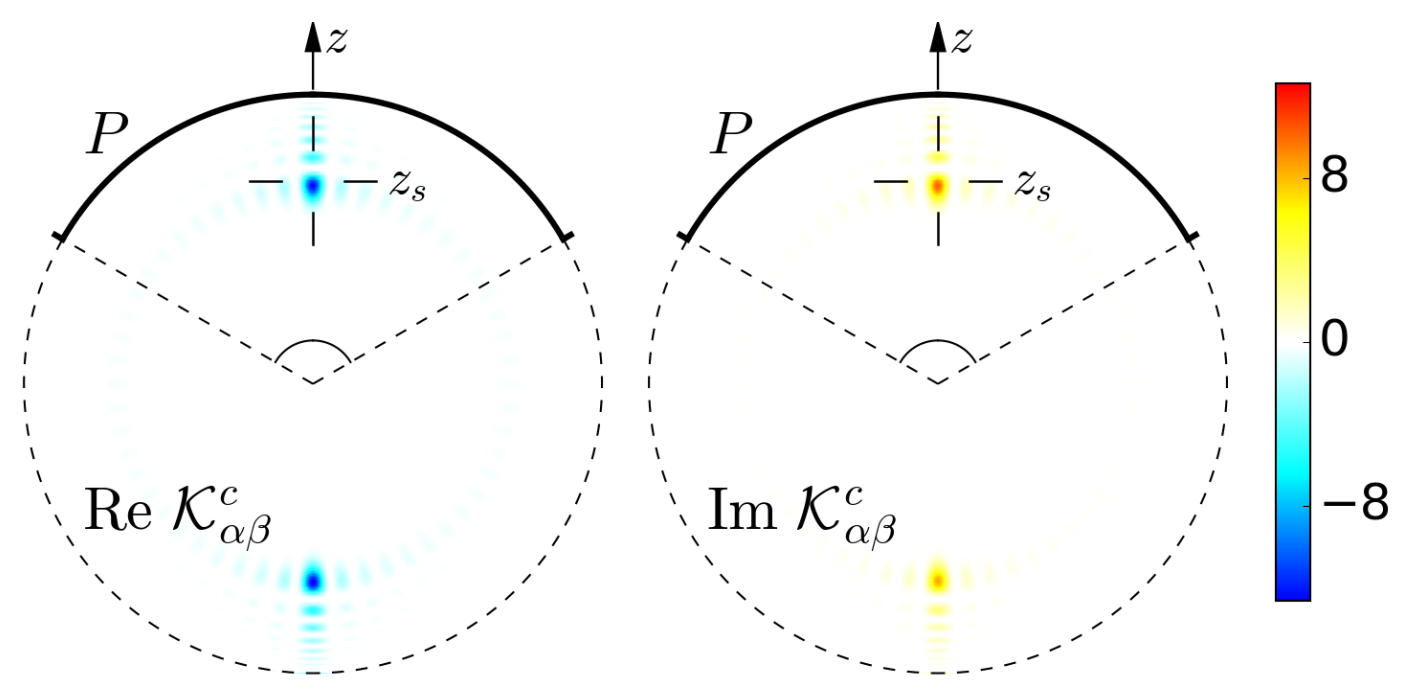

Figure 3.9: Meridional slice of the sound-speed kernel $\mathcal{K}_{\alpha \beta}^{c}$ with $z_{\mathrm{s}}=0.7 \mathrm{R}_{\odot}$ computed at the low frequency of $2.4000 \mathrm{mHz}$, which corresponds to the spike with a red dot in Fig. 3.7. This kernel displays oscillations and is not peaked as much as the $3-\mathrm{mHz}$ kernel from Fig. 3.2 . 
Our synthetic data do not contain a convective background. The effect of this background on signal-to-noise ratios in holography should be studied. Future work should also investigate the performance of PB holography for target locations that are away from the axis of the pupil, especially for farside imaging applications.

\section{Acknowledgements}

The theoretical framework was developed by L.G. and D.F. at Mathematisches Forschungsinstitut Oberwolfach Oberwolfach in May 2017. The numerical computations were performed by D.Y. using the Montjoie solver. D.Y. is a member of the International Max Planck Research School for Solar System Science at the University of Göttingen. We thank M. Duruflé and J. Chabassier from Inria Magique-3D for the helioseismologyrelated developments of Montjoie. We also thank Chris Hanson from NYUAD for the fine tuning of the model power spectrum of solar oscillations. L.G. acknowledges support from NYUAD Institute grant G1502. The computing resources were provided in part by the German Data Center for SDO, a project funded by the German Aerospace Center (DLR). 



\section{Imaging solar subsurface flows using diffraction-limited seismic holography}

\subsection{Abstract}

Helioseismic holography enables us to probe flows at targeted locations in the solar interior. Traditionally, directional phase shifts are measured using pupils with a quadrant geometry (method \#1). Another possibility is to correlate the reconstructed wave field at two nearby locations in the interior without changing the pupil (method \#2). In this chapter we implement both methods using simulated data and compare them. In doing so we use realistic numerical Green's functions for a standard solar model. The spatial resolution and signal-to-noise ratio is estimated for each method and each horizontal flow component at different depths in the convection zone. We find that method \#2 is consistently superior to method \#1 as it reaches the optimal spatial resolution (half the wavelength) and it has a higher signal-to-noise ratio. Furthermore method \#2 is much less subject to bias due to leakage from the solar surface. Method \#2 is very elegant and promising: we intend to use it in future applications of helioseismic holography to image solar subsurface flows.

\subsection{Introduction}

Understanding the structure and evolution of the Sun's large-scale flows is of great importance for solving the solar dynamo problem, specifically, where and how the magnetic field is generated and maintained in the Sun (see, e.g., Charbonneau 2014, Cameron et al. 2017). Helioseismology, which aims at deciphering the Sun's three-dimensional structure information encoded in the acoustic and surface gravity waves observed at the surface, is the only technique that can probe the Sun's internal flows (see, e.g., Gizon and Birch 2005, Basu 2016). Despite the huge quantity of helioseismic data obatained in the last two decades, the structure and evolution of the large-scale flows in the solar interior remain elusive. An improvement of flow-measuring strategies to interpret helioseismic measurements is needed in order to deal with inherent difficulties of the Sun such as the strong stratification near the solar surface or the high noise level due to granulation.

Helioseismic holography is one branch of helioseismology. These methods numerically focuses the observed acoustic waves into any desired location of the Sun to study 
the structure therein (see, e.g., Lindsey and Braun 1997, Yang 2018, Gizon et al. 2018). Two different strategies have been proposed to probe horizontal flows in the solar interior:

\#1 Lateral-vantage holography uses different pupil geometries (see, e.g., Lindsey and Braun 2004), such as quadrant pairs (Braun et al. 2004), to estimate forward and backward propagating waves at a given target location. The difference between time lags of waves traveling from the two pupils to the target point is sensitive to horizontal flows near the target location, hence a good diagnostic of subsurface horizontal flows (also see Section 4.4). Lateral-vantage holography has been used to measure both the small-scale (see, e.g., Braun et al. 2004, 2007) and large-scale (Braun and Birch 2008a) subsurface flows in the Sun.

\#2 Based on an analogy with time-distance helioseismology, Lindsey and Braun (1997) proposed to correlate two nearby points of estimated waves from holography to probe flows along the direction connecting the two points. This holographic correlation should behave in the same manner as the covariance function of waves, therefore is an extension of time-distance helioseismology in three dimensions (see Section 4.5).

Lindsey and Braun (1997) predicted that method \#2, hereafter the holographic correlation, would better measure flows than the lateral-vantage holography. However, the sensitivity of this measurement to flows has never been examined theoretically nor tested on observations. This article compares flow-sensitivity kernels and signal-to-noise ratios between the two flow-measuring strategies following the framework developed by Gizon et al. (2018) to define the signal and noise.

\subsection{Helioseismic holography to measure flows}

The Porter-Bojarski integral is defined as (Porter and Devaney 1982),

$$
\Phi_{\alpha}(\boldsymbol{r}, P):=\int_{P}\left[\psi\left(\boldsymbol{r}^{\prime}\right) \partial_{n} H_{\alpha}\left(\boldsymbol{r}^{\prime}, \boldsymbol{r}\right)-H_{\alpha}\left(\boldsymbol{r}^{\prime}, \boldsymbol{r}\right) \partial_{n} \psi\left(\boldsymbol{r}^{\prime}\right)\right] \mathrm{d} \boldsymbol{r}^{\prime},
$$

where $P$ denotes a pupil of the partially visible solar surface, $H_{\alpha}$ an acoustic wave propagator and $\psi$ the wavefield. Different choices of wave propagators and pupils give sensitivity to different quantities (see Table 2 in Gizon et al.2018).

Following Gizon et al. (2018), we suppose that the wavefield $\psi$ satisfies a Helmholtzlike equation,

$$
-\left(\Delta+k^{2}\right) \psi-\frac{2 \mathrm{i} \omega}{\rho^{1 / 2} c} \rho \boldsymbol{u} \cdot \nabla\left(\frac{\psi}{\rho^{1 / 2} c}\right)=s,
$$

where $\boldsymbol{u}$ is a steady flow, and $s$ is a source term that describes the stochastic excitation of the waves. This equation is solved at constant frequency $\omega$ in the computational domain $V$, with the reference density $\rho$ and sound-speed $c$ taken from a standard solar model. The local wavenumber $k(\boldsymbol{r}, \omega)$ is defined as

$$
k^{2}=\frac{\left(\omega^{2}+2 \mathrm{i} \omega \gamma\right)-\omega_{\mathrm{c}}^{2}}{c^{2}}, \quad \omega_{\mathrm{c}}^{2}=\rho^{1 / 2} c^{2} \Delta\left(\rho^{-1 / 2}\right),
$$

where $\boldsymbol{r}$ denotes the spatial position $(\boldsymbol{r} \in V), \gamma(\omega)$ is the damping rate that describes wave attenuation, and $\omega_{\mathrm{c}}(\boldsymbol{r})$ is the acoustic cut-off frequency. We solve Eq. $4.2 \mathrm{using}$ the same damping rate and source-covariance function as in Gizon et al. (2018), and the 
Sommerfeld-like radiation boundary condition (Atmo RBC 1) derived by Barucq et al. (2018).

We generalize the definition of holographic image intensity from Gizon et al. (2018) by allowing different focal points $\boldsymbol{r}$ and $\boldsymbol{r}^{\prime}$ for the two PB integrals $\Phi_{\alpha}$ and $\Phi_{\beta}$,

$$
I_{\alpha \beta}\left(\boldsymbol{r}, \boldsymbol{r}^{\prime}, P, P^{\prime}\right)=\Phi_{\alpha}^{*}(\boldsymbol{r}, P) \Phi_{\beta}\left(\boldsymbol{r}^{\prime}, P^{\prime}\right) .
$$

Separating the two focal points was first suggested by Lindsey and Braun (1997) but never studied.

Using the Born approximation, we decompose the holographic image intensity as the sum of a reference in a medium devoid of scatterers $I_{\alpha \beta, 0}$ and a small perturbation due to scatterers $\delta I_{\alpha \beta}$. The expectation value of $\delta I_{\alpha \beta}$ due to scatterers can be formulated as

$$
\begin{aligned}
\mathbb{E}\left[\delta I_{\alpha \beta}\right]= & \int K_{\alpha \beta}^{u_{r}} u_{r} d V+\int K_{\alpha \beta}^{u_{\theta}} u_{\theta} d V+\int K_{\alpha \beta}^{u_{\phi}} u_{\phi} d V \\
& +\int K_{\alpha \beta}^{c} \delta c d V+\int K_{\alpha \beta}^{\rho} \delta \rho d V,
\end{aligned}
$$

where $K_{\alpha \beta}^{u_{r}}, K_{\alpha \beta}^{u_{\theta}}, K_{\alpha \beta}^{u_{\phi}}, K_{\alpha \beta}^{c}$, and $K_{\alpha \beta}^{\rho}$ are sensitivity kernel functions of $u_{r}, u_{\theta}, u_{\phi}, \delta c$, and $\delta \rho$, whereas their expressions can be obtained following the derivations from Gizon et al. (2018).

The holographic measurement is sensitive to all scatterers and it is desirable to distinguish for example the flow and sound-speed contributions. This distinction is possible as flows are directional perturbations contrary to sound speed. In the following, we examine two measurements sensitive to flows while reducing the contributions from nondirectional perturbations.

\subsection{Quadrant geometry}

Lateral-vantage holography enhances contributions from flows by using two different pupils for the two PB integrals $\Phi_{\alpha}$ and $\Phi_{\beta}$. The pupil geometries are designed using the ray theory of wave propagation (see Figure 1 in Gizon et al. (2018) and the left panels of Fig. 4.1). For example, pupils in the east and west directions are chosen in order to be sensitive to the longitudinal component of the flow. In order to remove contributions from non-directional scatterers, we consider a difference between two measurements,

$$
I_{\alpha \beta}^{\mathrm{LV}}\left(\boldsymbol{r}, Q_{1}, Q_{2}\right)=\delta I_{\alpha \beta}\left(\boldsymbol{r}, \boldsymbol{r}, Q_{1}, Q_{2}\right)-\delta I_{\alpha \beta}\left(\boldsymbol{r}, \boldsymbol{r}, Q_{2}, Q_{1}\right)
$$

where the second measurement is done by flipping the two pupils. Note that the focal point is chosen to be same for the two PB integrals $\Phi_{\alpha}$ and $\Phi_{\beta}$.

\subsection{Holographic correlation}

An alternative way to enhance the contribution from flows is to use two different focal points $\boldsymbol{r}_{1}$ and $\boldsymbol{r}_{2}$ for the two PB integrals $\Phi_{\alpha}$ and $\Phi_{\beta}$ (see discussions in Lindsey and Braun 1997). The holographic correlation $I_{\alpha \beta}\left(\boldsymbol{r}_{1}, \boldsymbol{r}_{2}, P, P\right)$ is sensitive to flows between the 

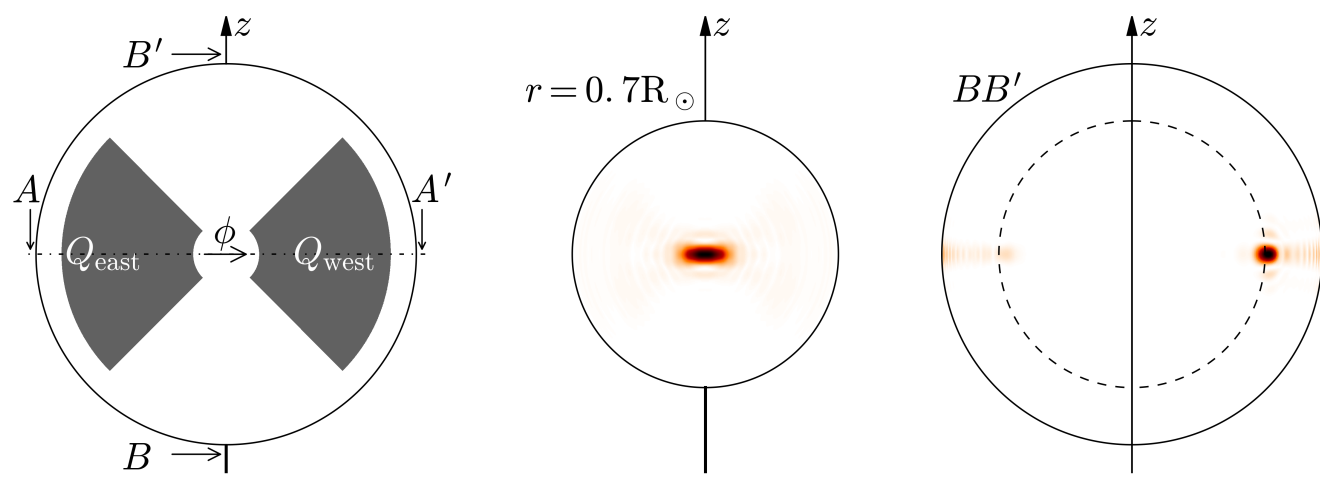

1.00
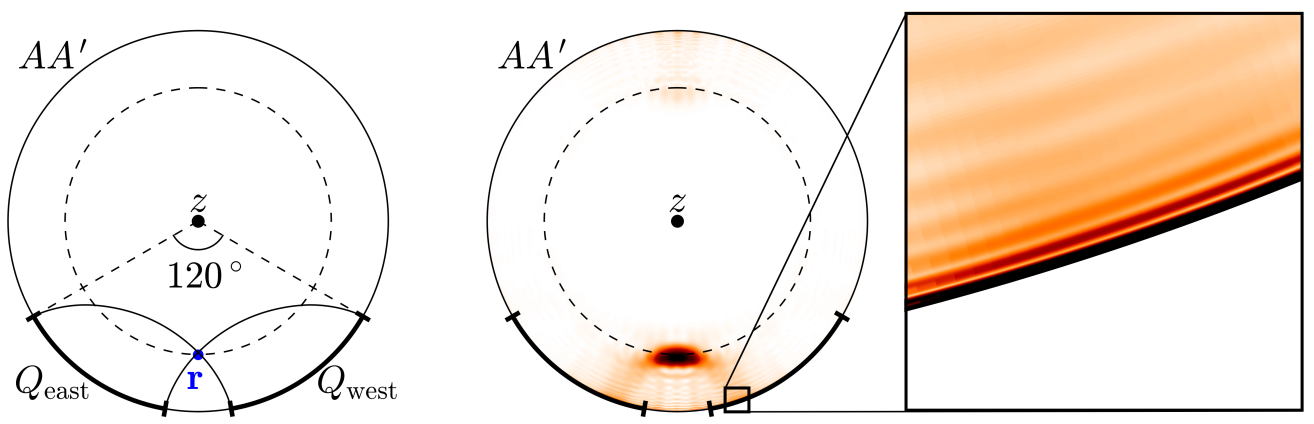

Figure 4.1: Kernel function $\mathcal{K}_{\alpha \beta}^{u_{\phi}}$ computed with the pupil geometry shown on the left panels that targets a flow along the $\phi$ direction at the bottom of convection zone (location $\mathbf{r}$ marked by a blue dot). The modulus of the kernel function is shown after averaging over 101 frequencies equally spaced from 2.75 to $3.25 \mathrm{mHz}$. Furthermore, the results are divided by the expectation value of the reference auto-correlation function of observed waves at $3 \mathrm{mHz}$, such that the holographic image intensity is dimensionless. The top panel shows a projection of the kernel on the sphere $r=0.7 \mathrm{R}_{\odot}$ (middle) and a 2D slice along the central meridian (right), whereas the bottom panel shows a 2D slice through the equator (middle) with a zoom near the surface (marked by the rectangle) shown on the right. The units are $10^{-4} \mathrm{~s} \mathrm{Mm}^{-4}$.

two focal points $\boldsymbol{r}_{1}$ and $\boldsymbol{r}_{2}$. This is a direct analogy with time-distance helioseismology but in the three-dimensional space. Specifically, $I_{\alpha \beta}\left(\boldsymbol{r}_{1}, \boldsymbol{r}_{2}, P, P\right)$ is an estimation of the cross-covariance function $C\left(\boldsymbol{r}_{1}, \boldsymbol{r}_{2}\right)$ between the two focal points $\boldsymbol{r}_{1}$ and $\boldsymbol{r}_{2}$, and hence $I_{\alpha \beta}\left(\boldsymbol{r}_{1}, \boldsymbol{r}_{2}, P, P\right)$ is sensitive to scatterers in the same manner as $\delta C\left(\boldsymbol{r}_{1}, \boldsymbol{r}_{2}\right)$. The pupil can simply be chosen as a cap of angular size $120^{\circ}$ (the pole of the cap is at the disc center) so that all the available data are used. As this measurement is sensitive to all scatterers, we define a difference measurement

$$
I_{\alpha \beta}^{\mathrm{HC}}(\boldsymbol{r}, \Delta \boldsymbol{r}, P)=\delta I_{\alpha \beta}\left(\boldsymbol{r}_{1}, \boldsymbol{r}_{2}, P, P\right)-\delta I_{\alpha \beta}\left(\boldsymbol{r}_{2}, \boldsymbol{r}_{1}, P, P\right)
$$

where $\boldsymbol{r}_{1}=\boldsymbol{r}-\frac{\Delta \boldsymbol{r}}{2}$ and $\boldsymbol{r}_{2}=\boldsymbol{r}+\frac{\Delta \boldsymbol{r}}{2}$

Hence, $I_{\alpha \beta}^{\mathrm{HC}}(\boldsymbol{r}, \Delta \boldsymbol{r}, P)$ should be mostly sensitive to flows along the direction $\Delta \boldsymbol{r}=\boldsymbol{r}_{2}-\boldsymbol{r}_{1}$. 

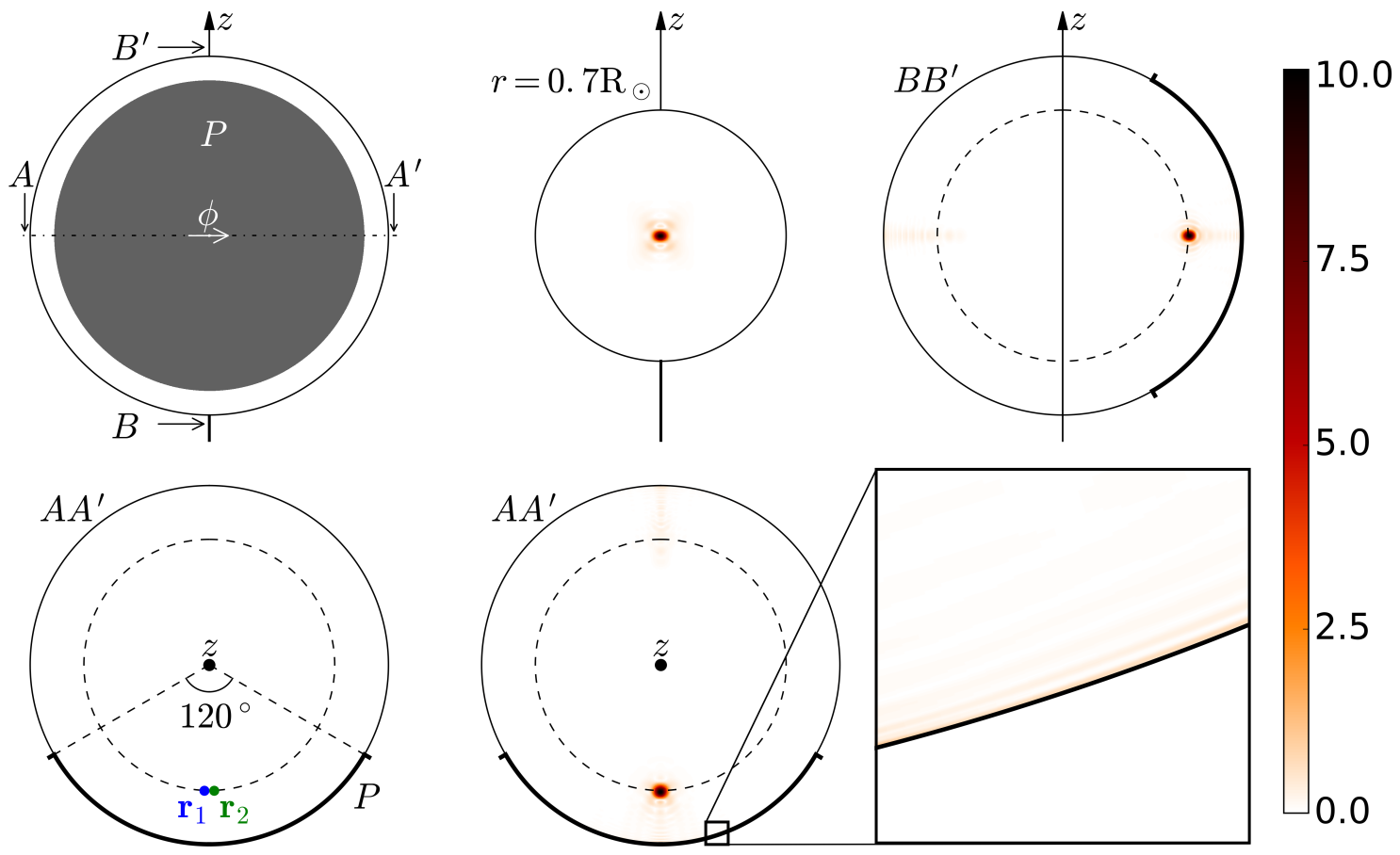

Figure 4.2: Same as Figure 4.1, but for the holographic correlation. The two focal points $\boldsymbol{r}_{1}$ and $\boldsymbol{r}_{2}$ are separated by half the local wavelength with locations indicated in the geometry plot on the left (blue and green dots). Compared to Fig. 4.1, the kernel function is more compact and suffers less contribution from the surface.

\subsection{Example computations}

\subsubsection{Zonal flow}

Figure 4.1 shows a flow kernel $\mathcal{K}_{\alpha \beta}^{u_{\phi}}$ for the lateral-vantage holography that averaged over 101 equally spaced frequencies from 2.75 to $3.25 \mathrm{mHz}$ targeting a longitudinal flow at $0.7 \mathrm{R}_{\odot}$. The pupils and wave propagators are taken from Table 2 of Gizon et al. (2018). We find that $\mathcal{K}_{\alpha \beta}^{u_{\phi}}$ is sensitive to flows near the focal point with a longitudinal resolution of 1.5 times the local wavelength and a latitudinal and radial resolution of half a wavelength. In this setup, the longitudinal resolution is far from optimal. However, some sensitivity of around $10 \%$ of the maximum value is observed between the target location and the surface and a strong contribution with an amplitude 1.5 times larger than at the target location comes from a thin layer slightly below the surface. All these artifacts could lead to a misinterpretation of the data.

Figure 4.2 shows an example flow kernel $\mathcal{K}_{\alpha \beta}^{u_{\phi}}$ for the holographic correlation targeting a depth of $0.7 \mathrm{R}_{\odot}$. The two focal points $\boldsymbol{r}_{1}$ and $\boldsymbol{r}_{2}$ are separated by half the local wavelength and chosen to be along the $\phi$ direction such that the measurement is sensitive to $u_{\phi}$. The wave propagators and the frequency range are the same as in Fig. 4.1. Compared to lateral-vantage holography, the kernel is more localized and the contribution from the near-surface region is significantly reduced ( $20 \%$ of the value at the target location).

Figure 4.3 shows a zoom of the kernel function $\mathcal{K}_{\alpha \beta}^{u_{\phi}}$ shown in Fig. 4.2 around the 
$A A^{\prime}$

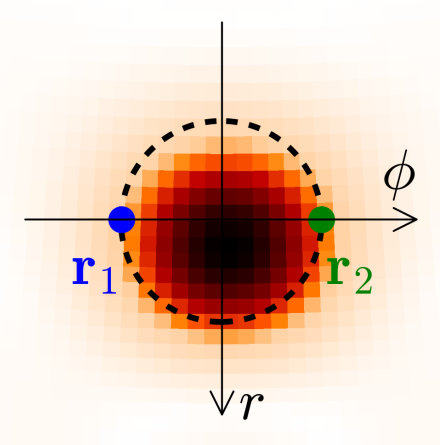

$B B^{\prime}$

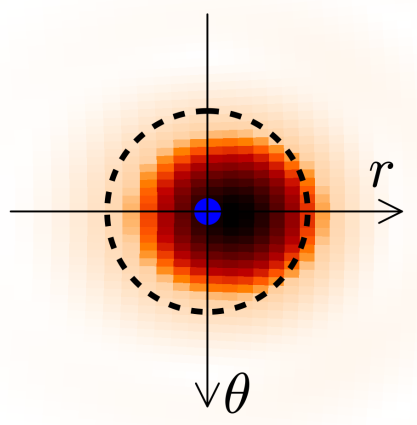

Figure 4.3: Zoom around the target location of the kernel function for $u_{\phi}$ shown in Fig. 4.2. The values are normalized to the maximum value of the kernel function. The blue and green dots mark the location of the two focal points. The circle has a diameter of half the local wavelength.

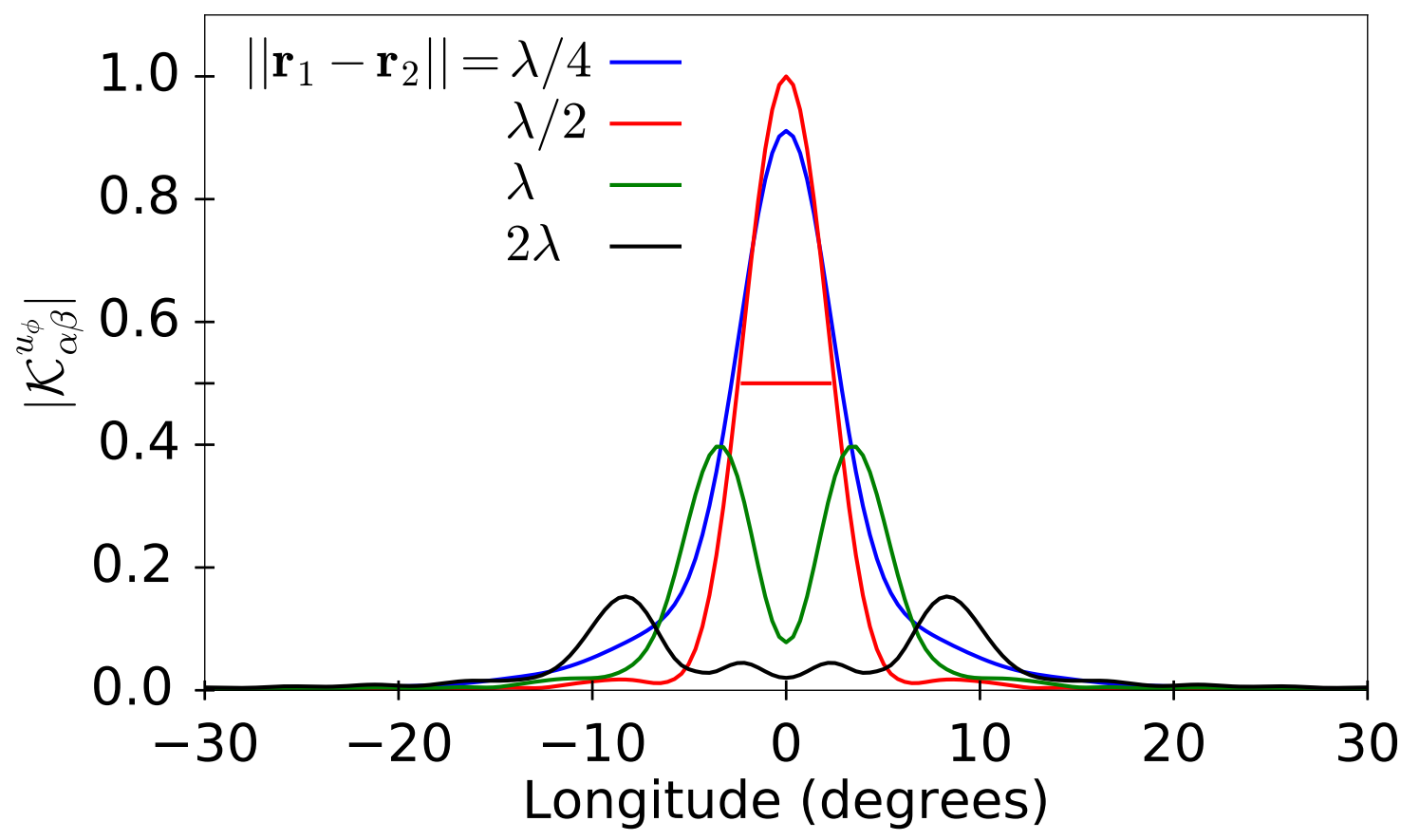

Figure 4.4: 1D slices of the kernel functions for $u_{\phi}$ along the $\phi$ coordinate using the geometry shown in Fig. 4.2. The separation distance between the two focal points $\mathbf{r}_{1}$ and $\mathbf{r}_{2}$ is chosen to be a quarter (blue), half (red), one (green), and two (black) local wavelengths $(\lambda)$. The red horizontal line is of size $\lambda / 2$, which is roughly the full width at half maximum of the kernel when the two focal points are separated by $\lambda / 2$. 
target location. We find that the kernel has a spatial resolution of roughly half the local wavelength along the radial and horizontal directions, which is the diffraction limit.

One would wonder what is the optimal separation distance between the two focal points $\boldsymbol{r}_{1}$ and $\boldsymbol{r}_{2}$. Figure 4.4 shows 1D slices of the kernel $\mathcal{K}_{\alpha \beta}^{u_{\phi}}$ along the $\phi$ direction using the geometry shown in Fig. 4.2 for different separation distances $\|\Delta \boldsymbol{r}\|$ between the two focal points $\boldsymbol{r}_{1}$ and $\boldsymbol{r}_{2}$. The kernel has the highest spatial resolution when the separation $\|\Delta \boldsymbol{r}\|$ is $\lambda / 2$, whereas $\|\Delta \boldsymbol{r}\|=\lambda / 4$ gives a slightly less compact kernel. When $\|\Delta \boldsymbol{r}\|$ is larger than $\lambda$, the kernel becomes most sensitive at the two focal points and is much smaller at the target location. Therefore, we fix a separation distance of $\lambda / 2$ between the two focal points in this study.

\subsubsection{Meridional flow}

Figure 4.5 shows examples of kernel functions targeting flows along the latitudinal direction using the holographic correlation. The two focal points are placed along the $\theta$ direction at $r=0.7 \mathrm{R}_{\odot}$ on the central meridian, and are separated by half the local wavelength. The left and middle panels show 2D slices of the kernel along the central meridian that target flows at latitude 30 and 60 degrees. The right panel shows 1D slices of these kernel functions along the $\phi$ direction, with the value normalized by their respective maxima. The localization of the kernel is as good at $30^{\circ}$ latitude as at the equator but it becomes worse at $60^{\circ}$. The kernels look very similar up to a latitude at $50^{\circ}$ (not shown here), i.e., as long as the two focal points have their latitude in the pupil range. The signal-to-noise ratio is roughly the same for all target locations up to $50^{\circ}$ latitude but becomes about three times smaller at 60 degrees.

Using lateral vantage holography, one would need to readjust pupils in order to target the correct latitude while the pupil is fixed to the full observational domain for the holographic correlation. This last method is thus more convenient.

\subsubsection{Signal-to-noise ratio}

A comparison of the kernel function between the two flow measuring strategies shows that the holographic correlation has a better spatial resolution than lateral-vantage holography. However, one needs to make sure that such a localized kernel is not at the cost of an increase of the noise level. In the following, we compare the signal-to-noise ratio of these two methods.

Following Gizon et al. (2018), we define the signal to be expectation values of Equation 4.6 and 4.7 due to flows, whereas the (realization) noise is due to the random wave excitation. In practice, holographic measurements are averaged over a range of frequencies $\left[\omega_{0}-\Delta \omega / 2, \omega_{0}+\Delta \omega / 2\right]$ to increase the signal-to-noise ratio. For an observation duration $T$, this interval contains $N=T \Delta \omega / 2 \pi$ independent frequencies. We define the signal-to-noise ratio as

$$
\mathrm{SNR}^{\mathrm{LV} / \mathrm{HC}}=\frac{\left|\mathbb{E}\left\langle I_{\alpha \beta}^{\mathrm{LV} / \mathrm{HC}}\right\rangle\right|}{\sqrt{\operatorname{Var}\left\langle I_{\alpha \beta, 0}^{\mathrm{LV} / \mathrm{HC}}\right\rangle}}=\frac{\sqrt{N}\left|\mathbb{E}\left\langle I_{\alpha \beta}^{\mathrm{LV} / \mathrm{HC}}\right\rangle\right|}{\sqrt{\left\langle\operatorname{Var} I_{\alpha \beta, 0}^{\mathrm{LV} / \mathrm{HC}}\right\rangle}},
$$



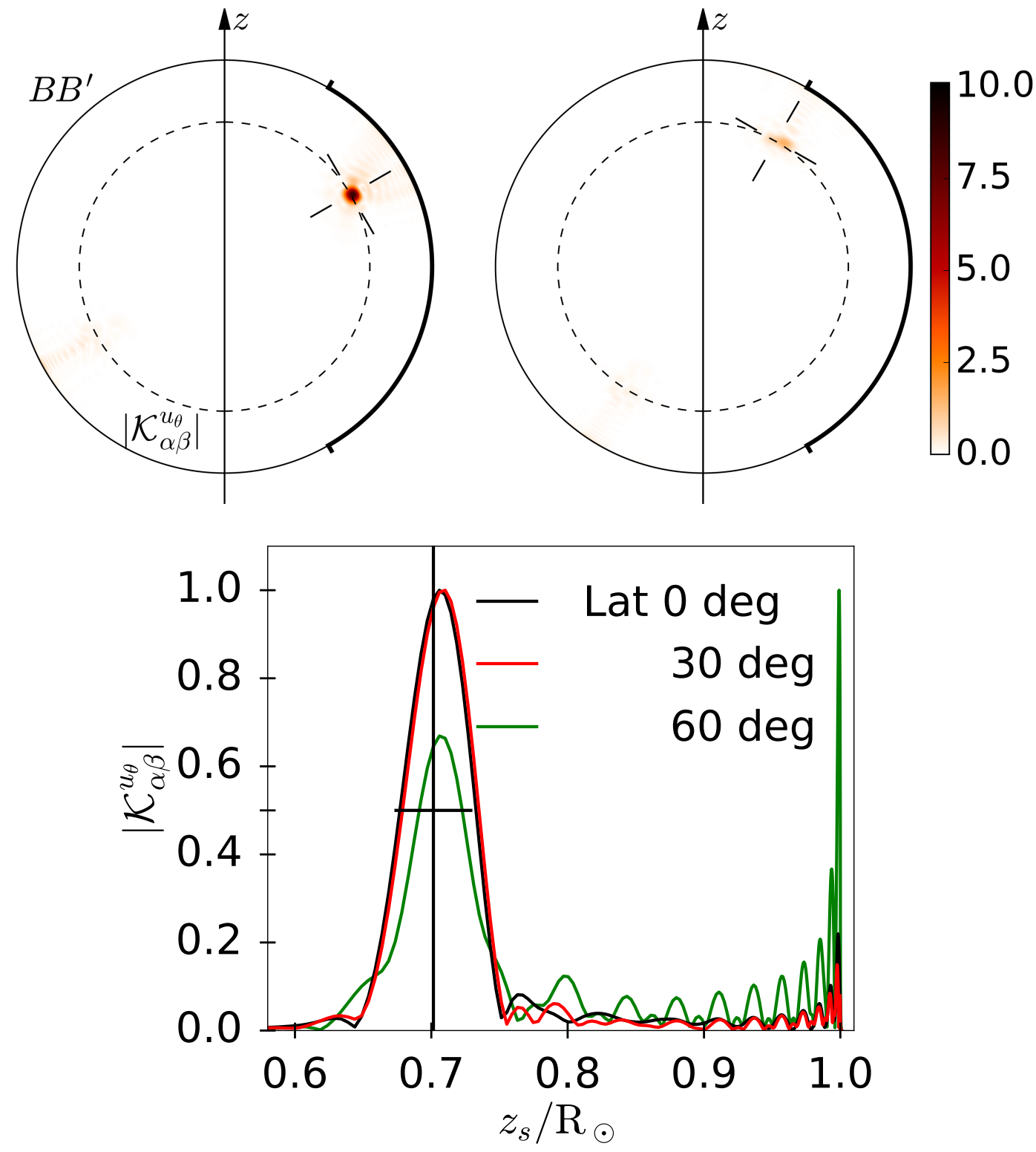

Figure 4.5: Kernel functions $\mathcal{K}_{\alpha \beta}^{u_{\theta}}$ for the holographic correlation targeting a flow $u_{\theta}$ at $0.7 \mathrm{R}_{\odot}$ on the central meridian at a latitude of $30^{\circ}$ (top left) and $60^{\circ}$ (top right). The target location is indicated by the black cross. The bottom panel shows normalized 1D slices of the kernel functions along the radial direction and passing through the target location at latitudes of 0 (black), 30 (red) and 60 (green) degrees. The horizontal line marks half the local wavelength. 


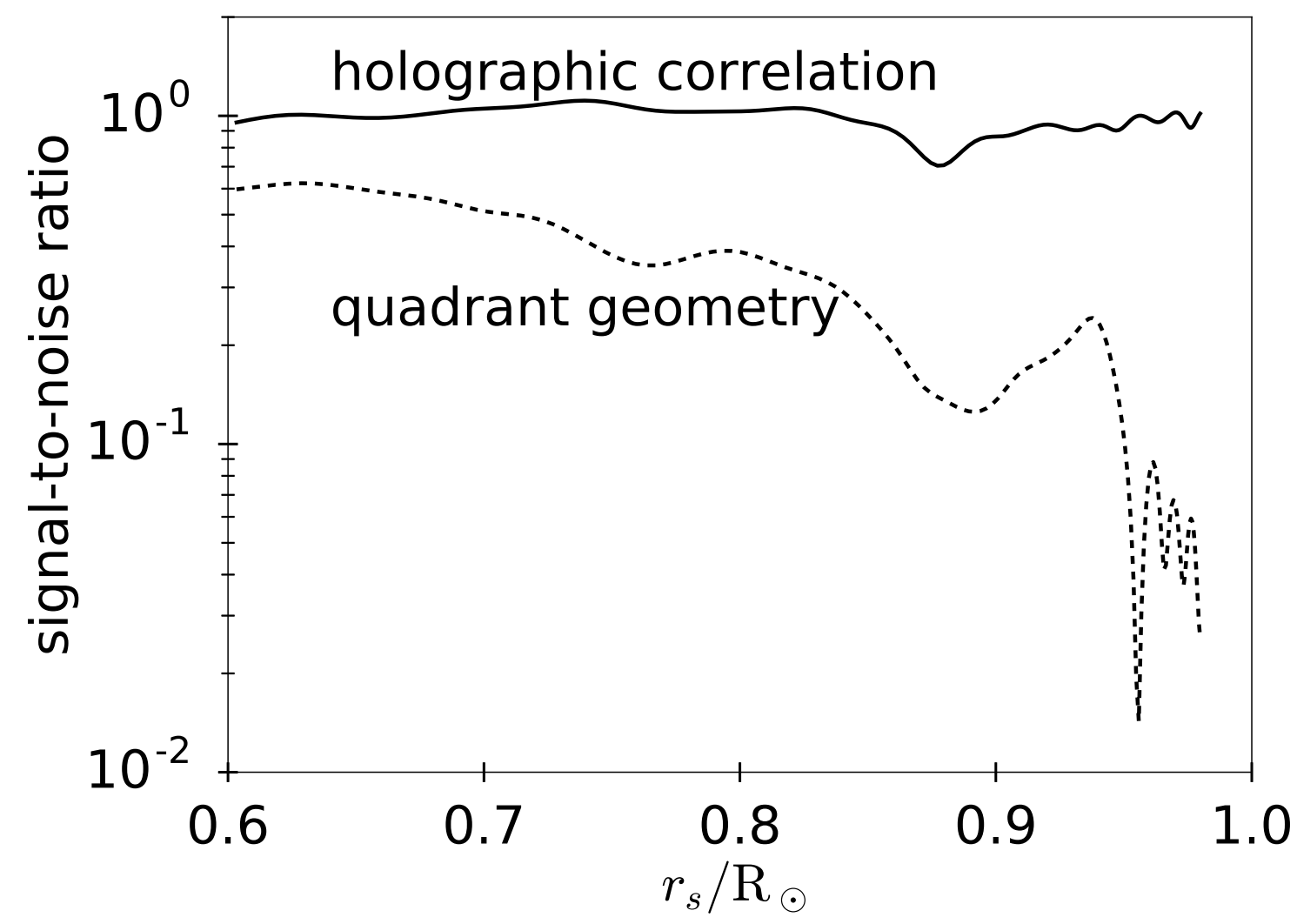

Figure 4.6: Signal-to-noise ratio as a function of depth for the two flow measuring strategies using the pupil geometries shown in Figs. 4.1 and 4.2. The signal is the longitudinal flow $u_{\phi}$ defined by Eq. 4.11

where the bracket denotes averaging of a given function $F$ in frequency

$$
\langle F\rangle=\frac{1}{N} \sum_{i=1}^{N} F\left(\omega_{i}\right) .
$$

The expression for the variance of the two measurements in the absence of scatterers can be obtained following derivations from Gizon et al. (2018). Note that $\sqrt{N}$ on the top of Equation (4.9) is due to the assumption that the noise at different frequencies is uncorrelated.

Figure 4.6 shows the signal-to-noise ratio (SNR) for the two strategies, for a localized longitudinal flow within a ball of diameter half the local wavelength. The amplitude of the flow is chosen to be $10 \%$ of the background sound-speed. The ball is placed at $\boldsymbol{r}_{\mathrm{s}}$ at different depths along the radial direction centered at the disc center. The signal is then approximated by

$$
\left|\mathbb{E}\left\langle I_{\alpha \beta}^{\mathrm{LV} / \mathrm{HC}}(\boldsymbol{r})\right\rangle\right| \simeq 0.1 c\left(\boldsymbol{r}_{\mathrm{s}}\right) V\left(\boldsymbol{r}_{\mathrm{s}}\right)\left|\mathcal{K}_{\alpha \beta}^{\mathrm{LV} / \mathrm{HC}, u_{\phi}}\left(\boldsymbol{r}, \boldsymbol{r}_{\mathrm{s}}\right)\right|, \quad V\left(\boldsymbol{r}_{\mathrm{s}}\right)=\frac{4 \pi}{3}\left(\frac{\lambda\left(\boldsymbol{r}_{\mathrm{s}}\right)}{4}\right)^{3} .
$$

The pupil geometries of the two methods are the same as in Fig. 4.1 (quadrant geometry) and 4.2 (holographic correlation), with the center of the two focal points located at $\boldsymbol{r}_{s}$. The 
holographic correlation possesses a SNR that is almost independent of depth and that is much higher than the SNR obtained using lateral-vantage holography. Thereby the better localization of the new holographic technique seen in Sect. 4.5 is not obtained at the price of a higher noise level but constitutes a real improvement of the measurement strategy.

\section{quadrant geometry}
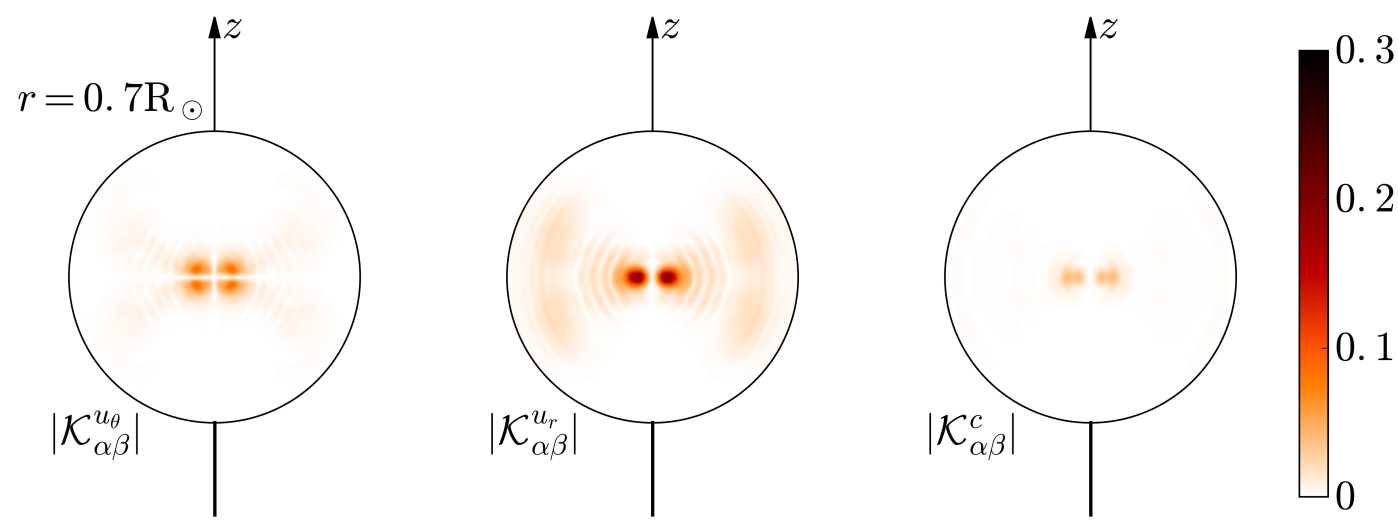

\section{holographic correlation}
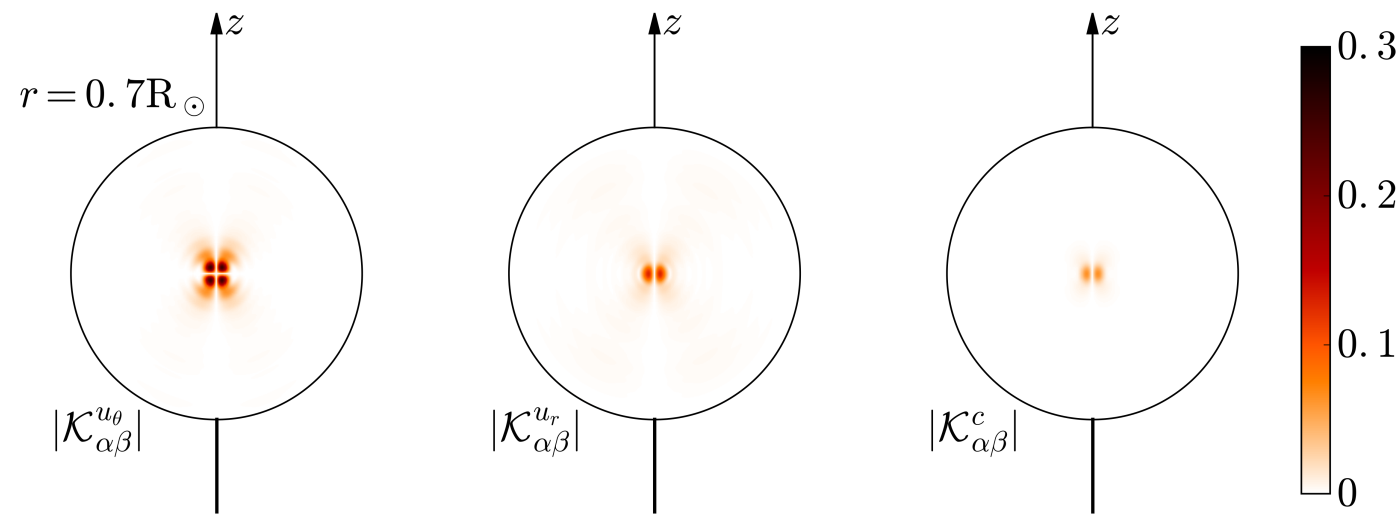

Figure 4.7: Sensitivity kernels for $u_{\theta}$ (left), $u_{r}$ (middle), and $c$ (right) with a measurement designed to be sensitive to $u_{\phi}$ (cross-talk kernels). The kernels are projected on the sphere of radius $r=0.7 \mathrm{R}_{\odot}$ and normalized to the maximum value of $\mathcal{K}_{\alpha \beta}^{u_{\phi}}\left(\boldsymbol{r}, \boldsymbol{r}_{\mathrm{s}}\right)$ shown in Figs. 4.1 (quadrant geometry) and 4.2 (holographic correlation). All kernel functions shown here are anti-symmetric with respect to the central meridian.

\subsection{Discussion}

\subsubsection{Cross-talk between zonal flow and other scatterers}

The measurement methods shown here aim at measuring flows in a particular direction. However, they may suffer from contributions due to flows in the other directions and 
sound-speed inhomogeneities.

Figure 4.7 shows kernel functions for $u_{\theta}, u_{r}$, and $c$ in the setups of Figs. 4.1 and 4.2 which were designed to be sensitive to $u_{\phi}$. For simplicity, we only show slices of a projection on the sphere of radius $r=0.7 \mathrm{R}_{\odot}$. We find in both methods non-zero crosscontributions from flows in the $\mathbf{r}$ and $\theta$ directions and sound-speed inhomogeneities, with amplitudes up to $30 \%$ of the maximum of the kernel for $u_{\phi}$. Therefore, caution should be taken in measuring subsurface flows. The kernel functions for undesired scatterers, however, are anti-symmetric with respect to the central meridian, which means that the crosscontribution is zero in the case of symmetric scatterers. Additionally, for azimuthally symmetric flows, an average of the measurements over different longitudes should reduce the cross-contributions from undesired scatterers and increase the signal-to-noise ratio.

\subsubsection{Outlook}

We found that holographic correlation better probes solar subsurface flows than the lateralvantage helioseismology. Specifically, it possesses more compact kernel functions and higher signal-to-noise ratio. Moreover, the measurement is more convenient as one does not need to change the pupils when changing the target location.

The next step is to apply this method to helioseismic observations in order to probe subsurface flows such as meridional circulation (Giles 2000, Liang et al. 2018b) or vortical flows (Langfellner et al. 2014). The current averaging schemes used in time-distance helioseismology to average the cross-covariance function can be directly extended to the three-dimensional space.

Furthermore, holographic measurements can be averaged over all longitudes to facilitate a direct comparison with global helioseismology as the same data are used. Such a comparison will allow to test the two helioseismic techniques, in particular, a validation of their treatments of the Sun's super-adiabatic layer which cannot be modelled properly at the moment. 



\section{Future work}

This dissertation developed the theoretical framework to evaluate signal and noise in helioseismic holography. Modeling experiments were conducted by solving a Helmholtzlike wave equation using the finite-element solver montjoie. The background coefficients are taken from a standard solar model. Our computations revealed that the PB integral is a better back-propagation method than the egression. Furthermore, the holographic correlation was found to possess a higher spatial resolution and signal-to-noise ratio than the quadrant geometry to measure the Sun's subsurface flows. All these findings can potentially improve the current imaging capability of the Sun's internal structure, thereby should be applied to helioseismic observations in future studies.

\subsection{Far-side imaging}

The framework developed in this dissertation can potentially improve far-side imaging, and hence facilitate a more accurate forecast of the space weather. Addionally, an application to far-side imaging is a good test of the new framework, since it is straightforward to determine whether it improves the current imaging capability of helioseismic holography.

In the following, I list limitations and proposed improvements to the current far-side technique:

1) The Green's function used in current far-side imaging pipeline is based on the ray theory of wave propagation, which is not valid for scatterers of size smaller than the first Fresnel zone. Green's functions that consider the finite wavelength of the waves should be implemented, in order to resolve more accurately the Sun's far-side structures with a higher spatial resolution. Such Green's functions can be obtained by solving a solar-like wave equation using the montjoie solver.

2) The egression is the current back-propagation method used in helioseismic holography. A replacement of the egression with the PB integral, which is a better backpropagation technique, should better locate far-side located scatterers with higher signalto-noise ratios.

3) Far-side helioseismic measurements are very noisy, whereby the current pipeline spatially averages the line-of-sight velocity field to boost signal-to-noise ratios. Such an averaging process reduces the computation cost to compute holograms, as less amount of data is transformed to the frequency space. However, it leads to loss of information contained in the raw data. Future work should take advantage of the advance of modern computational powers to compute holograms with all available data, and leave the averaging of holograms as a final step if necessary. This will allow more freedom to examine holographic measurements, which should improve its current imaging quality. 
4) The current far-side pipeline uses a two-skip Green's function to propagate waves. Zhao (2007) demonstrated that an inclusion of a three-skip Green's function can increase the signal-to-noise of far-side maps produced by time-distance helioseismology. Future study should investigate the potential improvement of combining multiple-skip Green's functions to image far-side active regions.

\subsection{Resolving solar internal flows at the diffraction limit}

An important finding of this dissertation is that the holographic correlation can resolve the Sun's subsurface horizontal flows to a spatial scale of half the local wavelength, whereas still possess higher signal-to-noise ratios than quadrant geometry. This means this new technique will probe the Sun's subsurface flows to the diffraction limit of acoustic waves, which has never been achieved before, whereby will largely improve our understanding of the structure and evolution of large-scale flows in the solar interior.

One potential application of the new flow-measuring strategy is to measure the Sun's rotation profile. The Sun's rotation rate has been measured by global helioseismology of its symmetric component of the two hemispheres. The PB holographic technique is able to measure both the symmetric and anti-symmetric components of solar differential rotation by averaging its measurements over all longitudes. This will help us to better understand the asymmetry in the rotation (and zonal flows) of the two hemispheres (see, e.g., Lekshmi et al.2018). Furthermore, it will allow a search for variations of longitudinal flows below the bottom of the convection zone, which is predicted by some hydrodynamic models to maintain the present structure of the tachocline (see, e.g., Spiegel and Zahn|1992, Dikpati and Gilman 1999).

Another application of the PB holographic technique is to probe the meridional circulation in the Sun's convection zone, which is crucial for some dynamo models to migrate the magnetic flux to lower latitudes during the solar cycle. Meridional circulation also redistributes the mass of the Sun, and hence its momentum, in the scale that is important for understanding the driving mechanism of Sun's differential rotation. Despite its importance, however, meridional circulation is notoriously difficult to detect due to its small amplitude in the solar interior, which is estimated to be a few $\mathrm{m} / \mathrm{s}$ at the bottom of the convection zone. The new flow-measuring strategy is much less susceptible to the noise than the current holographic technique, therefore is our best chance to probe meridional circulation in the solar interior. Furthermore, holographic measurements can be averaged over different longitudes, depths, and latitudes to boost signal-to-noise ratios, whereas the noise should decrease as square root of the number of uncorrelated measurements. 


\section{Bibliography}

Aerts, C., Christensen-Dalsgaard, J., Kurtz, D. W., 2010, Asteroseismology, Springer Science+Business Media B.V., ISBN 978-1-4020-5178-4

Aizenman, M. L., Smeyers, P., 1977, An analysis of the linear, adiabatic oscillations of a star in terms of potential fields, Ap\&SS, 48, 123-136

Appourchaux, T., Fröhlich, C., Andersen, B., Berthomieu, G., Chaplin, W. J., Elsworth, Y., Finsterle, W., Gough, D. O., Hoeksema, J. T., Isaak, G. R., Kosovichev, A. G., Provost, J., Scherrer, P. H., Sekii, T., Toutain, T., 2000, Observational Upper Limits to Low-Degree Solar g-Modes, ApJ, 538, 401-414

Bahcall, J., 2004, Solving the Mystery of the Missing Neutrinos, ArXiv Physics e-prints, physics $/ 0406040$

Barucq, H., Chabassier, J., Duruflé, M., Gizon, L., Leguèbe, M., 2018, Atmospheric radiation boundary conditions for the helmholtz equation, ESAIM: M2AN, 52, 945964

Basu, S., 2016, Global seismology of the Sun, Living Reviews in Solar Physics, 13, 2, 1606.07071

Besliu-Ionescu, D., Donea, A., Cally, P., 2017, Current State of Seismic Emission Associated with Solar Flares, Sun and Geosphere, 12, 59-67

Birch, A. C., Gizon, L., 2007, Linear sensitivity of helioseismic travel times to local flows, Astronomische Nachrichten, 328, 228, 1002.2338

Birch, A. C., Kosovichev, A. G., Price, G. H., Schlottmann, R. B., 2001, The Accuracy of the Born and Ray Approximations in Time-Distance Helioseismology, ApJ, 561, L229-L232

Birch, A. C., Braun, D. C., Hanasoge, S. M., Cameron, R., 2009, Surface-Focused Seismic Holography of Sunspots: II. Expectations from Numerical Simulations Using Sound-Speed Perturbations, Sol. Phys., 254, 17-27

Birch, A. C., Parchevsky, K. V., Braun, D. C., Kosovichev, A. G., 2011, "Hare and Hounds" Tests of Helioseismic Holography, Sol. Phys., 272, 11-28

Birch, A. C., Braun, D. C., Leka, K. D., Barnes, G., Javornik, B., 2013, Helioseismology of Pre-emerging Active Regions. II. Average Emergence Properties, ApJ, 762, 131, 1303.1391 
Birch, A. C., Schunker, H., Braun, D. C., Cameron, R., Gizon, L., Löptien, B., Rempel, M., 2016, A low upper limit on the subsurface rise speed of solar active regions, Science Advances, 2, e1600 557-e1600 557, 1607.05250

Bogart, R. S., Baldner, C., Basu, S., Haber, D. A., Rabello-Soares, M. C., 2011a, HMI ring diagram analysis I. The processing pipeline, GONG-SoHO 24: A New Era of Seismology of the Sun and Solar-Like Stars, 271, 012008

Bogart, R. S., Baldner, C., Basu, S., Haber, D. A., Rabello-Soares, M. C., 2011b, HMI ring diagram analysis II. Data products, GONG-SoHO 24: A New Era of Seismology of the Sun and Solar-Like Stars, 271, 012009

Born, M., Wolf, E., 1999, Principles of Optics, Cambridge University Press, Cambridge

Braun, D. C., 1995, Scattering of p-Modes by Sunspots. I. Observations, ApJ, 451, 859

Braun, D. C., 1997, Time-Distance Sunspot Seismology with GONG Data, ApJ, 487, $447-456$

Braun, D. C., 2014, Helioseismic Holography of an Artificial Submerged Sound Speed Perturbation and Implications for the Detection of Pre-emergence Signatures of Active Regions, Sol. Phys., 289, 459-474, 1210.7504

Braun, D. C., 2016, A Helioseismic Survey of Near-surface Flows Around Active Regions and their Association with Flares, ApJ, 819, 106, 1602.00038

Braun, D. C., Birch, A. C., 2008a, Prospects for the Detection of the Deep Solar Meridional Circulation, ApJ, 689, L161, 0810.0284

Braun, D. C., Birch, A. C., 2008b, Surface-Focused Seismic Holography of Sunspots: I. Observations, Sol. Phys., 251, 267-289, 0802.2652

Braun, D. C., Duvall, Jr., T. L., Labonte, B. J., 1987, Acoustic absorption by sunspots, ApJ, 319, L27-L31

Braun, D. C., Lindsey, C., Fan, Y., Jefferies, S. M., 1992, Local acoustic diagnostics of the solar interior, ApJ, 392, 739-745

Braun, D. C., Birch, A. C., Lindsey, C., 2004, Local Helioseismology of Near-Surface Flows, in SOHO 14 Helio- and Asteroseismology: Towards a Golden Future, (Ed.) D. Danesy, vol. 559 of ESA Special Publication, p. 337

Braun, D. C., Birch, A. C., Benson, D., Stein, R. F., Nordlund, Å., 2007, Helioseismic Holography of Simulated Solar Convection and Prospects for the Detection of SmallScale Subsurface Flows, ApJ, 669, 1395-1405, 0708.0214

Cameron, R. H., Dikpati, M., Brandenburg, A., 2017, The Global Solar Dynamo, Space Sci. Rev., 210, 367-395, 1602.01754

Chandrasekhar, S., 1964, A General Variational Principle Governing the Radial and the Non-Radial Oscillations of Gaseous Masses., ApJ, 139, 664 
Charbonneau, P., 2014, Solar Dynamo Theory, ARA\&A, 52, 251-290

Christensen-Dalsgaard, J., Däppen, W., Ajukov, S. V., Anderson, E. R., Antia, H. M., Basu, S., Batu rin, V. A., Berthomieu, G., Chaboyer, B., Chitre, S. M., Cox , A. N., Demarque, P., Donatowicz, J., Dziembowski, W. A., G abriel, M., Gough, D. O., Guenther, D. B., Guzik, J. A., Har vey, J. W., Hill, F., Houdek, G., Iglesias, C. A., Kosoviche v, A. G., Leibacher, J. W., Morel, P., Proffitt, C. R., Prov ost, J., Reiter, J., Rhodes, Jr., E. J., Rogers, F. J., Roxb urgh, I. W., Thompson, M. J., Ulrich, R. K., 1996, The Current State of Solar Modeling, Science, 272, 1286-1292

Deubner, F.-L., Gough, D., 1984, Helioseismology: Oscillations as a Diagnostic of the Solar Interior, ARA\&A, 22, 593-619

Devaney, A., Porter, R., 1985, Holography and the inverse source problem. part ii: Inhomogeneous media, JOSA A, 2, 2006-2012

Devaney, A. J., 2012, Mathematical Foundations of Imaging, Tomography and Wavefield Inversion, Cambridge University Press, Cambridge

Dikpati, M., Gilman, P. A., 1999, Joint Instability of Latitudinal Differential Rotation and Concentrated Toroidal Fields below the Solar Convection Zone, ApJ, 512, 417-441

Duruflé, M., 2006, Ph.D. thesis, ENSTA ParisTech, France

Duvall, Jr., T. L., Jefferies, S. M., Harvey, J. W., Pomerantz, M. A., 1993, Time-distance helioseismology, Nature, 362, 430-432

Edmonds, A. R., 1960, Angular Momentum in Quantum Mechanics, Princeton University Press, Princeton

Fossat, E., Boumier, P., Corbard, T., Provost, J., Salabert, D., Schmider, F. X., Gabriel, A. H., Grec, G., Renaud, C., Robillot, J. M., Roca-Cortés, T., Turck-Chièze, S., Ulrich, R. K., Lazrek, M., 2017, Asymptotic g modes: Evidence for a rapid rotation of the solar core, A\&A, 604, A40, 1708.00259

Fournier, D., Gizon, L., Hohage, T., Birch, A. C., 2014, Generalization of the noise model for time-distance helioseismology, A\&A, 567, A137, 1406.5335

Fournier, D., Leguèbe, M., Hanson, C. S., Gizon, L., Barucq, H., Chabassier, J., Duruflé, M., 2017, Atmospheric-radiation boundary conditions for high-frequency waves in time-distance helioseismology, A\&A, 608, A109, 1709.02156

Fournier, D., Hanson, C. S., Gizon, L., Barucq, H., 2018, Sensitivity kernels for timedistance helioseismology. Efficient computation for spherically symmetric solar models, A\&A, 616, A156, 1805.06141

Giles, P. M., 2000, Time-distance measurements of large-scale flows in the solar convection zone, Ph.D. thesis, STANFORD UNIVERSITY

Gizon, L., Birch, A. C., 2002, Time-distance helioseismology: the forward problem for random distributed sources, ApJ, 571, 966 
Gizon, L., Birch, A. C., 2004, Time-Distance Helioseismology: Noise Estimation, ApJ, $614,472-489$

Gizon, L., Birch, A. C., 2005, Local helioseismology, Living Reviews in Solar Physics, 2,6

Gizon, L., Duvall, T. L., Schou, J., 2003, Wave-like properties of solar supergranulation, Nature, 421, 43-44, astro-ph/0208343

Gizon, L., Schunker, H., Baldner, C. S., Basu, S., Birch, A. C., Bogart, R. S., Braun, D. C., Cameron, R., Duvall, T. L., Hanasoge, S. M., Jackiewicz, J., Roth, M., Stahn, T., Thompson, M. J., Zharkov, S., 2009, Helioseismology of Sunspots: A Case Study of NOAA Region 9787, Space Sci. Rev., 144, 249-273, 1002 . 2369

Gizon, L., Birch, A. C., Spruit, H. C., 2010, Local Helioseismology: Three-Dimensional Imaging of the Solar Interior, ARA\&A, 48, 289-338, 1001.0930

Gizon, L., Barucq, H., Duruflé, M., Hanson, C. S., Leguèbe, M., Birch, A. C., Chabassier, J., Fournier, D., Hohage, T., Papini, E., 2017, Computational helioseismology in the frequency domain: acoustic waves in axisymmetric solar models with flows, A\&A, 600, A 35

Gizon, L., Fournier, D., Yang, D., Birch, A. C., Barucq, H., 2018, Signal and noise in helioseismic holography, A\&A, 620, A136, 1810.00402

Goldreich, P., Keeley, D. A., 1977, Solar seismology. II - The stochastic excitation of the solar p-modes by turbulent convection, ApJ, 212, 243-251

Hanasoge, S., 2018, Measurement Process and Inversions Using Helioseismic Normalmode Coupling, ApJ, 861, 46, 1802.02776

Hanasoge, S., Mandal, K., 2019, Detection of Rossby Waves in the Sun using Normalmode Coupling, ApJ, 871, L32

Hanson, C. S., Donea, A. C., Leka, K. D., 2015, Enhanced Acoustic Emission in Relation to the Acoustic Halo Surrounding Active Region 11429, Sol. Phys., 290, 2171-2187, 1507.03447

Hartlep, T., Zhao, J., Mansour, N. N., Kosovichev, A. G., 2008, Validating Time-Distance Far-Side Imaging of Solar Active Regions through Numerical Simulations, ApJ, 689, $1373-1378,0805.0472$

Hill, F., 1988, Rings and trumpets - Three-dimensional power spectra of solar oscillations, ApJ, 333, 996-1013

Jackson, D. R., Dowling, D. R., 1991, Phase conjugation in underwater acoustics, Acoust. Soc. America J., 89, 171-181

Kuperman, W. A., Hodgkiss, W. S., Song, H. C., Akal, T., Ferla, C., Jackson, D. R., 1998, Phase conjugation in the ocean: Experimental demonstration of an acoustic timereversal mirror, Acoust. Soc. America J., 103, 25-40 
Lamb, H., 1909, On Kinetic Stability, Proc. London Math. Soc., 7, 122

Landau, L. D., Lifshitz, E. M., 1975, The classical theory of fields, Pergamon Press, Oxford

Langfellner, J., Gizon, L., Birch, A. C., 2014, Time-distance helioseismology: A new averaging scheme for measuring flow vorticity, A\&A, 570, A90, 1408.4669

Langfellner, J., Birch, A. C., Gizon, L., 2018, Evolution and wave-like properties of the average solar supergranule, A\&A, 617, A97, 1805.12522

Larson, T. P., Schou, J., 2018, Global-Mode Analysis of Full-Disk Data from the Michelson Doppler Imager and the Helioseismic and Magnetic Imager, Sol. Phys., 293, 29

Leighton, R. B., Noyes, R. W., Simon, G. W., 1962, Velocity Fields in the Solar Atmosphere. I. Preliminary Report., ApJ, 135, 474

Lekshmi, B., Nandy, D., Antia, H. M., 2018, Asymmetry in Solar Torsional Oscillation and the Sunspot Cycle, ApJ, 861, 121

Liang, Z.-C., Gizon, L., Birch, A. C., Duvall, Jr, T. L., 2018a, Time-distance helioseismology of solar Rossby waves, arXiv e-prints, 1812.07413

Liang, Z.-C., Gizon, L., Birch, A. C., Duvall, Jr., T. L., Rajaguru, S. P., 2018b, Solar meridional circulation from twenty-one years of SOHO/MDI and SDO/HMI observations: Helioseismic travel times and forward modeling in the ray approximation, ArXiv e-prints, 1808.08874

Liewer, P. C., González Hernández, I., Hall, J. R., Lindsey, C., Lin, X., 2014, Testing the Reliability of Predictions of Far-Side Active Regions from Helioseismology Using STEREO Far-Side Observations of Solar Activity, Sol. Phys., 289, 3617-3640

Lindsey, C., Braun, D. C., 1990, Helioseismic imaging of sunspots at their antipodes, Sol. Phys., 126, 101-115

Lindsey, C., Braun, D. C., 1997, Helioseismic Holography, ApJ, 485, 895-903

Lindsey, C., Braun, D. C., 2000a, Basic principles of solar acoustic holography-(invited review), Sol. Phys., 192, 261-284

Lindsey, C., Braun, D. C., 2000b, Seismic Images of the Far Side of the Sun, Science, 287, 1799-1801

Lindsey, C., Braun, D. C., 2004, Principles of Seismic Holography for Diagnostics of the Shallow Subphotosphere, ApJS, 155, 209-225

Lindsey, C., Braun, D. C., 2005a, The Acoustic Showerglass. I. Seismic Diagnostics of Photospheric Magnetic Fields, ApJ, 620, 1107-1117

Lindsey, C., Braun, D. C., 2005b, The Acoustic Showerglass. II. Imaging Active Region Subphotospheres, ApJ, 620, 1118-1131 
Lindsey, C., Birch, A. C., Donea, A.-C., 2006, Simulations of Acoustic Excitation, in Solar MHD Theory and Observations: A High Spatial Resolution Perspective, (Eds.) J. Leibacher, R. F. Stein, H. Uitenbroek, vol. 354 of Astronomical Society of the Pacific Conference Series, p. 174

Lindsey, C., Braun, D., Hernández, I. G., Donea, A., 2011, Computational Seismic Holography of Acoustic Waves in the Solar Interior, in Holography - Different Fields of Application, (Ed.) F. Monroy Ramirez

Löptien, B., Birch, A. C., Gizon, L., Schou, J., Appourchaux, T., Blanco Rodríguez, J., Cally, P. S., Dominguez-Tagle, C., Gandorfer, A., Hill, F., Hirzberger, J., Scherrer, P. H., Solanki, S. K., 2015, Helioseismology with Solar Orbiter, Space Sci. Rev., 196, 251-283, 1406.5435

Löptien, B., Gizon, L., Birch, A. C., Schou, J., Proxauf, B., Duvall, T. L., Bogart, R. S., Christensen, U. R., 2018, Global-scale equatorial Rossby waves as an essential component of solar internal dynamics, Nature Astronomy, 2, 568-573, 1805.07244

Müller, D., Marsden, R. G., St. Cyr, O. C., Gilbert, H. R., 2013, Solar Orbiter . Exploring the Sun-Heliosphere Connection, Sol. Phys., 285, 25-70, 1207.4579

Munk, J. M., 2001, Helioseismic Time-Distance Inversion, Ph.D. thesis, University of Aarhus, Aarhus, Denmark, iSBN:87-90400-25-9

Pérez Hernández, F., González Hernández, I., 2010, Green's Functions for Far-Side Seismic Images: A Polar-Expansion Approach, ApJ, 711, 853-860, 1003.1103

Porter, R. P., 1969, Image formation with arbitrary holographic type surfaces, Physics Letters A, 29, 193-194

Porter, R. P., Devaney, A. J., 1982, Holography and the inverse source problem, JOSA, 72, 327-330

Pourabdian, M., Fournier, D., Gizon, L., 2018, Comparison of Travel-Time and Amplitude Measurements for Deep-Focusing Time-Distance Helioseismology, Sol. Phys., 293, 66, 1804.02311

Ruzmaikin, A., Lindsey, C., 2003, Helioseismic probing of the solar dynamo, in GONG+ 2002. Local and Global Helioseismology: the Present and Future, (Ed.) H. SawayaLacoste, vol. 517 of ESA Special Publication, pp. 71-74

Schou, J., 2015, Effects of granulation on the visibility of solar oscillations, A\&A, 580, L11, 1507.08856

Schou, J., Antia, H. M., Basu, S., Bogart, R. S., Bush, R. I., Chitre, S. M., ChristensenDalsgaard, J., Di Mauro, M. P., Dziembowski, W. A., Eff-Darwich, A., Gough, D. O., Haber, D. A., Hoeksema, J. T., Howe, R., Korzennik, S. G., Kosovichev, A. G., Larsen, R. M., Pijpers, F. P., Scherrer, P. H., Sekii, T., Tarbell, T. D., Title, A. M., Thompson, M. J., Toomre, J., 1998, Helioseismic Studies of Differential Rotation in the Solar Envelope by the Solar Oscillations Investigation Using the Michelson Doppler Imager, ApJ, 505, 390-417 
Schunker, H., Schou, J., Gaulme, P., Gizon, L., 2018, Fragile Detection of Solar g-Modes by Fossat et al., Sol. Phys., 293, 95, 1804.04407

Sekii, T., Appourchaux, T., Fleck, B., Turck-Chièze, S., 2015, Future Mission Concepts for Helioseismology, Space Sci. Rev., 196, 285-302

Skartlien, R., 2001, Imaging of Acoustic Wave Sources inside the Sun, ApJ, 554, 488-495

Skartlien, R., 2002, Local Helioseismology as an Inverse Source-Inverse Scattering Problem, ApJ, 565, 1348-1365

Song, H. C., Kuperman, W. A., Hodgkiss, W. S., 1998, A time-reversal mirror with variable range focusing, Acoust. Soc. America J., 103, 3234-3240

Spiegel, E. A., Zahn, J.-P., 1992, The solar tachocline, A\&A, 265, 106-114

Stein, R. F., Nordlund, Å., 2001, Solar Oscillations and Convection. II. Excitation of Radial Oscillations, ApJ, 546, 585-603, astro-ph/0008048

Stix, M., 2002, The sun: an introduction, Springer, Berlin

Tsang, L., Ishimaru, A., Porter, R. P., Rouseff, D., 1987, Holography and the inverse source problem. iii. inhomogeneous attenuative media, JOSA A, 4, 1783-1787

Unno, W., Osaki, Y., Ando, H., Saio, H., Shibahashi, H., 1989, Nonradial oscillations of stars, University of Tokyo Press, Tokyo

Woch, J., Gizon, L., 2007, The Solar Orbiter mission and its prospects for helioseismology, Astron. Nach., 328, 362, 1002.2278

Woodard, M., Schou, J., Birch, A. C., Larson, T. P., 2013, Global-Oscillation Eigenfunction Measurements of Solar Meridional Flow, Sol. Phys., 287, 129-147

Woodard, M. F., 2016, Evidence for large-scale subsurface convection in the Sun, MNRAS, 460, 3292-3297, 1605.06192

Yang, D., 2018, Ghost Images in Helioseismic Holography? Toy Models in a Uniform Medium, Sol. Phys., 293, 17, 1801.01759

Zhao, J., 2007, Time-Distance Imaging of Solar Far-Side Active Regions, ApJ, 664, L139-L142, 0706.2886

Zharkov, S., Green, L. M., Matthews, S. A., Zharkova, V. V., 2013, Properties of the 15 February 2011 Flare Seismic Sources, Sol. Phys., 284, 315-327, 1208.4284 

Appendix 



\section{A Computing PB integrals in spherical harmonic space}

\section{A.1 Notations}

We use spherical coordinate $\boldsymbol{r}=(r, \theta, \phi)$ and we denote $\hat{\boldsymbol{r}}$ as the unit vector along the direction of $\boldsymbol{r}$.

\section{A.2 Spherical harmonics}

Spherical harmonics are defined as

$$
\begin{aligned}
Y_{l}^{m}(\theta, \phi) & =c_{l}^{m} P_{l}^{m}(\cos \theta) \exp (\mathrm{i} m \phi), \\
c_{l}^{m} & =\sqrt{\frac{2 l+1}{4 \pi} \frac{(l-m) !}{(l+m) !}},
\end{aligned}
$$

where $P_{l}^{m}$ is the associated Legendre polynomials with

$$
\int_{-1}^{1} \mathrm{~d} x P_{l}^{m}(x) P_{k}^{m}(x)=\frac{2(l+m) !}{(2 l+1)(l-m) !} \delta_{k, l} .
$$

When $m=0$, spherical harmonics are related to the normalized Legendre polynomials $\mathcal{P}_{l}$ by

$$
Y_{l}^{0}(\theta, \phi)=\sqrt{\frac{1}{2 \pi}} \mathcal{P}_{l}(\cos \theta) .
$$

\section{A.3 Rotating axis-symmetric functions with spherical har- monics}

For any function $f\left(\boldsymbol{r}, \boldsymbol{r}^{\prime}\right)$ that depends only on $r, r^{\prime}$, and $\hat{\boldsymbol{r}} \cdot \hat{\boldsymbol{r}}^{\prime}$, it can be written into sums of spherical harmonics

$$
f\left(\boldsymbol{r}, \boldsymbol{r}^{\prime}\right)=\sum_{l=0}^{\infty} \sum_{m=-l}^{l} F_{l}^{m}\left(\boldsymbol{r}, r^{\prime}\right) Y_{l}^{m}\left(\hat{\boldsymbol{r}}^{\prime}\right) .
$$


When $\boldsymbol{r}$ is along the polar axis $\hat{z}, f$ can be written as a sum of normalized Legendre polynomials

$$
f\left(r \hat{z}, \boldsymbol{r}^{\prime}\right)=\sqrt{\frac{1}{2 \pi}} \sum_{l=0}^{\infty} \mathcal{F}_{l}\left(r, r^{\prime}\right) \mathcal{P}_{l}\left(\cos \theta^{\prime}\right) .
$$

We note that the factor $\sqrt{1 / 2 \pi}$ is added here such that Equation $(A .5)$ is consistent with Equation (A.4), which is due to the relation between spherical harmonics and normalized Legendre function (see Equation A.3).

By using the rotation formula of spherical harmonics, $F_{l}^{m}$ can be rewritten as

$$
F_{l}^{m}\left(\boldsymbol{r}, r^{\prime}\right)=\sqrt{\frac{4 \pi}{2 l+1}} \mathcal{F}_{l}\left(r, r^{\prime}\right) Y_{l}^{m *}(\hat{\boldsymbol{r}}),
$$

and replacing $F_{l}^{m}$ with the above formula, Equation $\mathrm{A} .5$ becomes

$$
f\left(\boldsymbol{r}, \boldsymbol{r}^{\prime}\right)=\sum_{l=0}^{\infty} \sum_{m=-l}^{l} \sqrt{\frac{4 \pi}{2 l+1}} \mathcal{F}_{l}\left(r, r^{\prime}\right) Y_{l}^{m *}(\hat{\boldsymbol{r}}) Y_{l}^{m}\left(\hat{\boldsymbol{r}}^{\prime}\right) .
$$

Equation A.7 can be simplified using the addition theorem of spherical harmonics

$$
\begin{aligned}
\mathcal{P}_{l}(\cos \alpha) & =\sqrt{\frac{8 \pi^{2}}{2 l+1}} \sum_{m=-l}^{l} Y_{l}^{m *}(\hat{\boldsymbol{r}}) Y_{l}^{m}\left(\hat{\boldsymbol{r}}^{\prime}\right), \\
\cos \alpha & =\cos \theta \cos \theta^{\prime}+\cos \left(\phi-\phi^{\prime}\right) \sin \theta \sin \theta^{\prime} .
\end{aligned}
$$

It leads to

$$
f\left(\boldsymbol{r}, \boldsymbol{r}^{\prime}\right)=\sqrt{\frac{1}{2 \pi}} \sum_{l=0}^{\infty} \mathcal{F}_{l}\left(r, r^{\prime}\right) \mathcal{P}_{l}(\cos \alpha) .
$$

\section{A.4 Source-sensitivity kernel in spherical harmonic space}

In this section, we compute the reference source-sensitivity kernel of the PB integral in a medium devoid of sound-speed scatterers and flows with the Green's function (and wave propagator) excited by a source fixed along the polar axis. This is achieved by projecting the Green's function into spherical harmonic space and then rotating to the desired location.

First, we rewrite the reference source-sensitivity kernel function $\left[K_{A, 0}^{\mathrm{PB}}\right]($ Eq. (3.25)) as

$$
K_{A, 0}^{\mathrm{PB}}\left(\boldsymbol{r}, \boldsymbol{r}_{s}\right)=\oint_{\partial \odot} \mathrm{d} \boldsymbol{r}^{\prime} w_{A}\left(\boldsymbol{r}^{\prime}\right)\left\{G_{0}\left(\boldsymbol{r}^{\prime}, \boldsymbol{r}_{s}\right) \partial_{n^{\prime}} H_{0}\left(\boldsymbol{r}, \boldsymbol{r}^{\prime}\right)-H_{0}\left(\boldsymbol{r}, \boldsymbol{r}^{\prime}\right) \partial_{n^{\prime}} G_{0}\left(\boldsymbol{r}^{\prime}, \boldsymbol{r}_{s}\right)\right\}
$$

where $\boldsymbol{r}$ and $\boldsymbol{r}_{s}$ are target and source locations, $\boldsymbol{r}^{\prime}$ is located at the solar surface $\partial \odot$, and $w_{A}$ is the window function that is equal to one within the pupil $A$ and zero otherwise. This form of the kernel function grants a general way of computing $K_{A}^{\mathrm{PB}}$ in the spherical harmonic space with arbitrary coverage $A$. 
Then we decompose all functions on the right hand side of Equation A.10 into spherical harmonics,

$$
\begin{aligned}
G_{0}\left(\boldsymbol{r}_{s}, \boldsymbol{r}^{\prime}\right) & =\sum_{l_{1}=0}^{\infty} \sum_{m_{1}=-l_{1}}^{l_{1}} \sqrt{\frac{4 \pi}{2 l_{1}+1}} \mathcal{G}_{l_{1}}\left(r_{s}, \mathrm{R}_{\odot}\right) Y_{l_{1}}^{m_{1} *}\left(\hat{\boldsymbol{r}}_{s}\right) Y_{l_{1}}^{m_{1}}\left(\hat{\boldsymbol{r}}^{\prime}\right), \\
H_{0}\left(\boldsymbol{r}, \boldsymbol{r}^{\prime}\right) & =\sum_{l_{2}=0}^{\infty} \sum_{m_{2}=-l_{2}}^{l_{2}} \sqrt{\frac{4 \pi}{2 l_{2}+1}} \mathcal{H}_{l_{2}}\left(r, \mathrm{R}_{\odot}\right) Y_{l_{2}}^{m_{2} *}(\hat{\boldsymbol{r}}) Y_{l_{2}}^{m_{2}}\left(\hat{\boldsymbol{r}}^{\prime}\right), \\
w_{A}\left(\boldsymbol{r}^{\prime}\right) & =\sum_{l_{3}=0}^{\infty} \sum_{m_{3}=-l_{3}}^{l_{3}} W_{l_{3}}^{m_{3}} Y_{l_{3}}^{m_{3}}\left(\hat{\boldsymbol{r}}^{\prime}\right) .
\end{aligned}
$$

Using seismic reciprocity $G_{0}\left(\boldsymbol{r}^{\prime}, \boldsymbol{r}_{s}\right)=G_{0}\left(\boldsymbol{r}_{s}, \boldsymbol{r}^{\prime}\right)$, and inserting Equation (A.11) into Equation A.10, one obtains

$$
\begin{aligned}
K_{A, 0}^{\mathrm{PB}}\left(\boldsymbol{r}, \boldsymbol{r}_{s}\right) & =\sum_{l_{1}=0}^{\infty} \sqrt{\frac{4 \pi}{2 l_{1}+1}} \sum_{l_{2}=0}^{\infty} \sqrt{\frac{4 \pi}{2 l_{2}+1}}\left\{\mathcal{G}_{l_{1}}\left(r_{s}, \mathrm{R}_{\odot}\right) \partial_{n^{\prime}} \mathcal{H}_{l_{2}}\left(r, \mathrm{R}_{\odot}\right)-\mathcal{H}_{l_{2}}\left(r, \mathrm{R}_{\odot}\right) \partial_{n^{\prime}} \mathcal{G}_{l_{1}}\left(r_{s}, \mathrm{R}_{\odot}\right)\right\} \\
& \left.\times \sum_{m_{1}=-l_{1}}^{l_{1}} \sum_{m_{2}=-l_{2}}^{l_{2}} \sum_{l_{3}=0}^{\infty} \sum_{m_{3}=-l_{3}}^{l_{3}} W_{l_{3}}^{m_{3}} Y_{l_{1}}^{m_{1} *}\left(\hat{\boldsymbol{r}}_{s}\right) Y_{l_{2}}^{m_{2} *}(\hat{\boldsymbol{r}}) \oint_{\partial \odot} \mathrm{d} \boldsymbol{r}^{\prime} Y_{l_{1}}^{m_{1}}\left(\hat{\boldsymbol{r}}^{\prime}\right) Y_{l_{2}}^{m_{2}}\left(\hat{\boldsymbol{r}}^{\prime}\right) Y_{l_{3}}^{m_{3}}\left(\hat{\boldsymbol{r}}^{\prime}\right) . \quad \text { (A. } 12\right)
\end{aligned}
$$

The surface integral $\oint_{\partial \odot} \mathrm{d} \boldsymbol{r}^{\prime} Y_{l_{1}}^{m_{1}}\left(\hat{\boldsymbol{r}}^{\prime}\right) Y_{l_{2}}^{m_{2}}\left(\hat{\boldsymbol{r}}^{\prime}\right) Y_{l_{3}}^{m_{3}}\left(\hat{\boldsymbol{r}}^{\prime}\right)$ on the right-hand side of the above equation is known as the Gaunt's formula, which can be computed analytically (see, e.g., Edmonds 1960, Eq. (4.6.3)).

\section{A.5 Noise in spherical harmonic space}

The noise due to stochastic nature of background sources can be rewritten as (see, e.g., Eq. (3.33))

$$
\begin{aligned}
& \mathbb{E}\left[\Phi_{\alpha, 0}^{\mathrm{PB} *}(\boldsymbol{r}) \Phi_{\beta, 0}^{\mathrm{PB}}\left(\boldsymbol{r}^{\prime}\right)\right]=\oint_{\partial \odot} \mathrm{d} \boldsymbol{r}_{1} w_{A, 1}\left(\boldsymbol{r}_{1}\right) \oint_{\partial \odot} \mathrm{d} \boldsymbol{r}_{2} w_{A, 2}\left(\boldsymbol{r}_{2}\right)\left\{C_{0}\left(\boldsymbol{r}_{1}, \boldsymbol{r}_{2}\right) \partial_{n_{1}} H_{\alpha, 0}^{*}\left(\boldsymbol{r}, \boldsymbol{r}_{1}\right) \partial_{n_{2}} H_{\beta, 0}\left(\boldsymbol{r}^{\prime}, \boldsymbol{r}_{2}\right)\right. \\
& -\left[\partial_{n_{2}} C_{0}\left(\boldsymbol{r}_{1}, \boldsymbol{r}_{2}\right)\right] \partial_{n_{1}} H_{\alpha, 0}^{*}\left(\boldsymbol{r}, \boldsymbol{r}_{1}\right) H_{\beta, 0}\left(\boldsymbol{r}^{\prime}, \boldsymbol{r}_{2}\right)-\left[\partial_{n_{1}} C_{0}\left(\boldsymbol{r}_{1}, \boldsymbol{r}_{2}\right)\right] H_{\alpha, 0}^{*}\left(\boldsymbol{r}, \boldsymbol{r}_{1}\right) \partial_{n_{2}} H_{\beta, 0}\left(\boldsymbol{r}^{\prime}, \boldsymbol{r}_{2}\right) \\
& \left.+\left[\partial_{n_{1}} \partial_{n_{2}} C_{0}\left(\boldsymbol{r}_{1}, \boldsymbol{r}_{2}\right)\right] H_{\alpha, 0}^{*}\left(\boldsymbol{r}, \boldsymbol{r}_{1}\right) H_{\beta, 0}\left(\boldsymbol{r}^{\prime}, \boldsymbol{r}_{2}\right)\right\},
\end{aligned}
$$

where $C$ denotes the cross-covariance function of the waves and $\boldsymbol{r}_{1}=\mathrm{R}_{\odot} \hat{\boldsymbol{r}}_{1}$ and $\boldsymbol{r}_{2}=$ $\mathrm{R}_{\odot} \hat{\boldsymbol{r}}_{2}$ are points located on the solar surface. Similar to the source-sensitivity kernel, all 
functions in Equation $\mathrm{A} .13$ can be decomposed into spherical harmonics,

$$
\begin{aligned}
C_{0}\left(\boldsymbol{r}_{1}, \boldsymbol{r}_{2}\right) & =\sum_{l_{1}=0}^{\infty} \sum_{m_{1}=-l_{1}}^{l_{1}} \sqrt{\frac{4 \pi}{2 l_{1}+1}} \mathscr{l}_{l_{1}}\left(\mathrm{R}_{\odot}, \mathrm{R}_{\odot}\right) Y_{l_{1}}^{m_{1} *}\left(\hat{\boldsymbol{r}}_{1}\right) Y_{l_{1}}^{m_{1}}\left(\hat{\boldsymbol{r}}_{2}\right), \\
H_{\alpha, 0}^{*}\left(\boldsymbol{r}, \boldsymbol{r}_{1}\right) & =\sum_{l_{2}=0}^{\infty} \sum_{m_{2}=-l_{2}}^{l_{2}} \sqrt{\frac{4 \pi}{2 l_{2}+1}} \mathcal{H}_{\alpha, l_{2}}^{*}\left(r, \mathrm{R}_{\odot}\right) Y_{l_{2}}^{m_{2}}(\hat{\boldsymbol{r}}) Y_{l_{2}}^{m_{2} *}\left(\hat{\boldsymbol{r}}_{1}\right), \\
H_{\beta, 0}\left(\boldsymbol{r}^{\prime}, \boldsymbol{r}_{2}\right) & =\sum_{l_{3}=0}^{\infty} \sum_{m_{3}=-l_{3}}^{l_{3}} \sqrt{\frac{4 \pi}{2 l_{3}+1}} \mathcal{H}_{\beta, l_{3}}\left(r^{\prime}, \mathrm{R}_{\odot}\right) Y_{l_{3}}^{m_{3} *}\left(\hat{\boldsymbol{r}}^{\prime}\right) Y_{l_{3}}^{m_{3}}\left(\hat{\boldsymbol{r}}_{2}\right), \\
w_{A, 1}\left(\boldsymbol{r}_{1}\right) & =\sum_{l_{4}=0}^{\infty} \sum_{m_{4}=-l_{4}}^{l_{4}} W_{1, l_{4}}^{m_{4}} Y_{l_{4}}^{m_{4}}\left(\hat{\boldsymbol{r}}_{1}\right), \\
w_{A, 2}\left(\boldsymbol{r}_{2}\right) & =\sum_{l_{5}=0}^{\infty} \sum_{m_{5}=-l_{5}}^{l_{5}} W_{2, l_{5}}^{m_{5}} Y_{l_{5}}^{m_{5}}\left(\hat{\boldsymbol{r}}_{2}\right) .
\end{aligned}
$$

Inserting Equation (A.14) into Equation A.13, one obtains

$$
\begin{aligned}
& \mathbb{E}\left[\Phi_{\alpha, 0}^{\mathrm{PB} *}(\boldsymbol{r}) \Phi_{\beta, 0}^{\mathrm{PB}}\left(\boldsymbol{r}^{\prime}\right)\right]=\sum_{l_{1}=0}^{\infty} \sqrt{\frac{4 \pi}{2 l_{1}+1}} \sum_{l_{2}=0}^{\infty} \sqrt{\frac{4 \pi}{2 l_{2}+1}} \sum_{l_{3}=0}^{\infty} \sqrt{\frac{4 \pi}{2 l_{3}+1}} \\
& \times\left\{\mathscr{C}_{l_{1}}\left(\mathrm{R}_{\odot}, \mathrm{R}_{\odot}\right) \partial_{n_{1}} \mathcal{H}_{\alpha, l_{2}}^{*}\left(r, \mathrm{R}_{\odot}\right) \partial_{n_{2}} \mathcal{H}_{\beta, l_{3}}\left(r^{\prime}, \mathrm{R}_{\odot}\right)-\partial_{n_{2}} \mathscr{C}_{l_{1}}\left(\mathrm{R}_{\odot}, \mathrm{R}_{\odot}\right) \partial_{n_{1}} \mathcal{H}_{\alpha, l_{2}}^{*}\left(r, \mathrm{R}_{\odot}\right) \mathcal{H}_{\beta, l_{3}}\left(r^{\prime}, \mathrm{R}_{\odot}\right)\right. \\
& \left.-\partial_{n_{1}} \mathscr{C}_{l_{1}}\left(\mathrm{R}_{\odot}, \mathrm{R}_{\odot}\right) \mathcal{H}_{\alpha, l_{2}}^{*}\left(r, \mathrm{R}_{\odot}\right) \partial_{n_{2}} \mathcal{H}_{\beta, l_{3}}\left(r^{\prime}, \mathrm{R}_{\odot}\right)+\partial_{n_{1}} \partial_{n_{2}} \mathscr{l}_{l_{1}}\left(\mathrm{R}_{\odot}, \mathrm{R}_{\odot}\right) \mathcal{H}_{\alpha, l_{2}}^{*}\left(r, \mathrm{R}_{\odot}\right) \mathcal{H}_{\beta, l_{3}}\left(r^{\prime}, \mathrm{R}_{\odot}\right)\right\} \\
& \times \sum_{m_{1}=-l_{1}}^{l_{1}} \sum_{m_{2}=-l_{2}}^{l_{2}} Y_{l_{2}}^{m_{2}}(\hat{\boldsymbol{r}}) \sum_{l_{4}=0}^{\infty} \sum_{m_{4}=-l_{4}}^{l_{4}} W_{1, l_{4}}^{m_{4}} \oint_{\partial \odot} \mathrm{d} \boldsymbol{r}_{1} Y_{l_{1}}^{m_{1} *}\left(\hat{\boldsymbol{r}}_{1}\right) Y_{l_{2}}^{m_{2} *}\left(\hat{\boldsymbol{r}}_{1}\right) Y_{l_{4}}^{m_{4}}\left(\hat{\boldsymbol{r}}_{1}\right) \\
& \times \sum_{m_{3}=-l_{3}}^{l_{3}} Y_{l_{3}}^{m_{3} *}\left(\hat{\boldsymbol{r}}^{\prime}\right) \sum_{l_{5}=0}^{\infty} \sum_{m_{5}=-l_{5}}^{l_{5}} W_{2, l_{5}}^{m_{5}} \oint_{\partial \odot} \mathrm{d} \boldsymbol{r}_{2} Y_{l_{1}}^{m_{1}}\left(\hat{\boldsymbol{r}}_{2}\right) Y_{l_{3}}^{m_{3}}\left(\hat{\boldsymbol{r}}_{2}\right) Y_{l_{5}}^{m_{5}}\left(\hat{\boldsymbol{r}}_{2}\right) .
\end{aligned}
$$




\section{Scientific contributions}

\section{Publications}

- Dan Yang: Ghost Images in Helioseismic Holography? Toy Models in a Uniform Medium, Solar Physics 293, 17, 2018

- Laurent Gizon, Damien Fournier, Dan Yang, Aaron C. Birch, and Hélène Barucq: Signal and noise in helioseismic holography, Astronomy \& Astrophysics, 620, 136, 2018

- S.K. Solanki et al. (including Dan Yang): The Polarimetric and Helioseismic Imager on Solar Orbiter, Astronomy \& Astrophysics (accepted), arXiv eprint:1903.11061

\section{Conference contributions}

- XXXth General Assembly of the IAU, Vienna, Austria, 20-31 August 2018 Oral Presentation: Helioseismic Holography Using Multiple Vantage Points

- Rocks \& Stars II, Göttingen, Germany, 13-16 September 2017

Oral Presentation: Comparisons between Helioseismic Holography and PorterBojarski Holography

- The many Scales of the Universe, Annual Meeting of the Astronomische Gesellschaft, Göttingen, Germany, 18-22 September 2017

Poster: A Possible Improvement on Helioseismic Holography

- Stellar and Planetary Dynamo, Göttingen, Germany, 26-29 May 2015

Poster: Helioseismic Holography: Fundamentals of Acoustic Holography 



\section{Acknowledgements}

First of all, I would like to thank my supervisor Laurent Gizon for guiding me from solving toy problems to real-life scientific problems, as well as his trust and patience during the last four years. I also thank Aaron C. Birch and Damien Fournier for their insights and fruitful discussions. This dissertation would not have been possible without their guidance and support.

I would also like to thank Chris and Michael for showing me the beauty of coding and writing, and Emanuele, Hannah, Jan, Jesper, Robert, Tom, and Zhichao for various discussions. Stefan Dreizler is thanked for being in my TAC and making sure that my $\mathrm{Ph} . \mathrm{D}$. was on the right path. Christoph Lehrenfeld, Hardi Peter, and Ramin Yahyapour are thanked for kindly joining the defense committee.

I am indebted to Hélène Barucq and the Inria group for developing the Montjoie solver, which is crucial for this dissertation. I also thank Bernard and Ray for answering cluster related questions.

I wish to thank the International Max Planck Research School (IMPRS) for Solar System Science and the Solar and Stellar Interiors department at the MPS for funding, and for creating a great atmosphere for Ph.D. studies. For future funding, I am grateful to Laurent for kindly offered me a postdoc position, which allows me to focus on the research.

Federico, Jörg, Matthias, Sarah, Sirila, Sonja, Vincent, and others in the department are thanked for the laughs, rumors/gossips, good (and bad) jokes, and, more importantly, cakes during coffee times on the green sofa area. I also thank the Chinese community at the MPS and IMPRS students for making me feel at home during these years.

I am grateful to my parents, my sister, and my niece for their love, encouragement, and support.

Finally, I would like to thank Göttingen for bringing the love of my life, 英晨, whom made my life much brighter.

感谢负晨照亮了我的人生。愿此爱永恒。 



\section{Curriculum vitae}

\section{Personal}

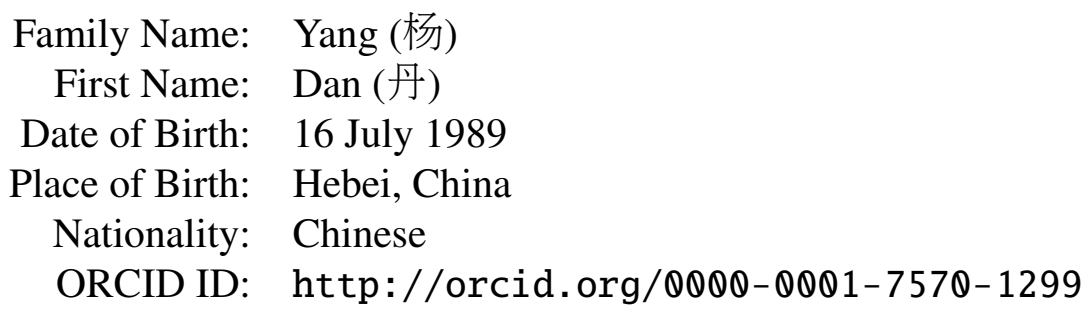

\section{Education}

- Ph.D. Student, International Max Planck Research School for Solar System Science at the University of Göttingen, Germany

November 2014 - December 2018

- Research project at the Max Planck Institute for Solar System Research, Germany Thesis: Modeling experiments in helioseismic holography

- M.Sc., Astrophysics, University of Chinese Academy of Sciences, China September 2011- July 2014

- Research project at Yunnan Observatories, Chinese Academy of Sciences, China Thesis: The observational study on bipolar emerging active regions and magnetic helicity fluxes at the photospheric level

- B.Sc., Physics, College of Physics Science \& Technology, Hebei University, China September 2007 - July 2011

Thesis: Geometrical diagnostic for spatial Ricci scalar dark energy model 